\title{
Critical Analysis of Different Hilbert-Huang Algorithms for Pavement Profile Evaluation
}

\author{
by \\ Yaw Okyere Adu-Gyamfi
}

A thesis submitted to the Faculty of the University of Delaware in partial

fulfillment of the requirements for the degree of Master of Degree in Civil Engineering

Spring 2010

Copyright 2009 Yaw Okyere Adu-Gyamfi

All Rights Reserved 


\title{
CRITICAL ANALYSIS OF DIFFERENT HILBERT-HUANG ALGORITHMS FOR PAVEMENT PROFILE EVALUATION
}

\author{
by \\ Yaw Okyere Adu-Gyamfi
}

Approved:

Busby N.O. Attoh-Okine, $\mathrm{PhD}$

Professor in charge of thesis on behalf of the Advisory Committee

Approved:

Harry (Tripp) Shenton III, $\mathrm{PhD}$

Chair of the Department of Civil Engineering

Approved:

Michael J. Chajes, PhD

Dean of the College of Engineering

Approved:

Debra Hess Norris, M.S

Vice Provost for Graduate and Professional Education 


\section{ACKNOWLEDGMENTS}

I specially thank God for His profound grace and guidance throughout this work. I sincerely thank and appreciate the contribution from all individuals who have made this work a success.

First and foremost, thanks to my graduate advisor, Prof. Nii Attoh Okine, for his invaluable guidance, patience, support and encouragement which has led to the successful completion of this thesis. I also wish to express my thanks to Dr. A.Y. Ayenu Prah for providing me with support and guidance.

My sincere gratitude goes out to the professors at University of Delaware for their teaching, academic advice, and support during my studies. I am always grateful to my parents and friends for being there for me. 


\section{TABLE OF CONTENTS}

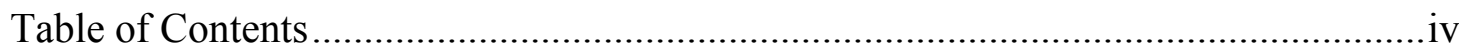

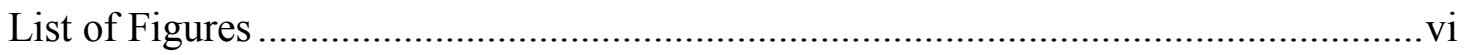

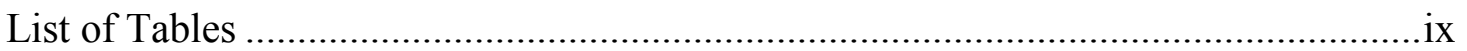

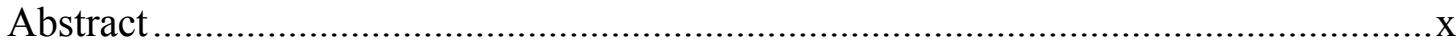

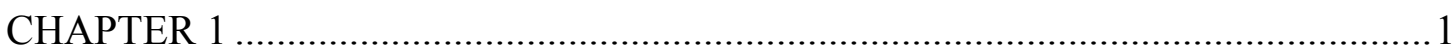

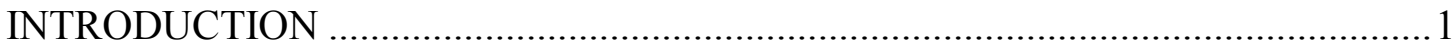

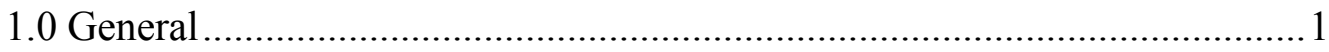

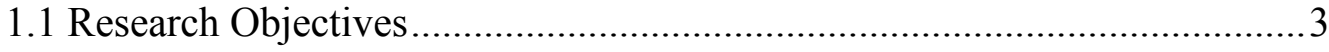

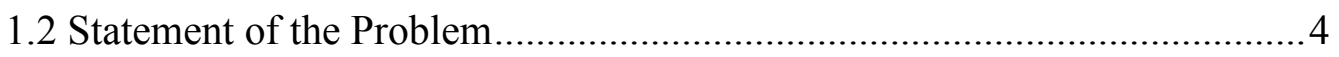

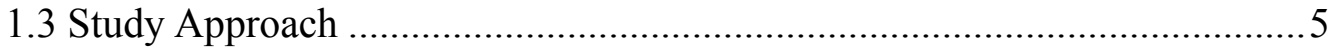

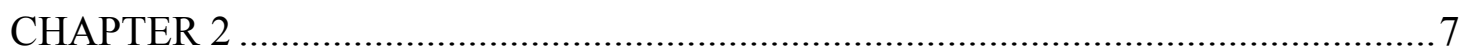

BACKGROUND AND LITERATURE REVIEW ..................................................

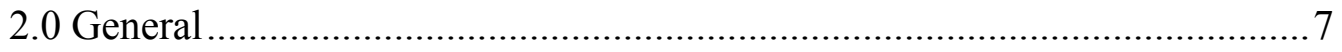

2.01 Non-Spectral Techniques...................................................................

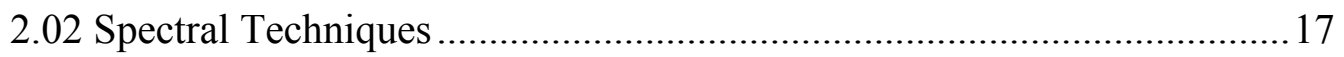

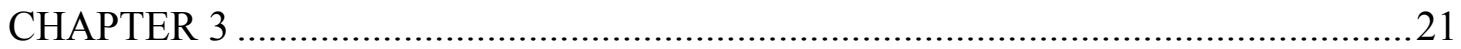

DEVELOPMENT OF THE METHODOLOGY ……................................................21 
3.0 The Hilbert-Huang Transform ...................................................... 21

3.1 Ensemble Empirical Mode Decomposition .........................................22

3.2 Complex Empirical Mode Decomposition (CEMD) ..............................29

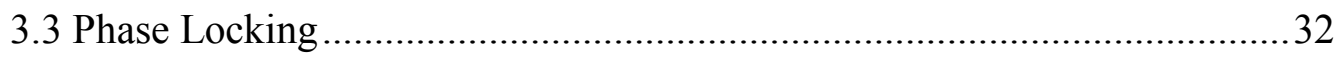

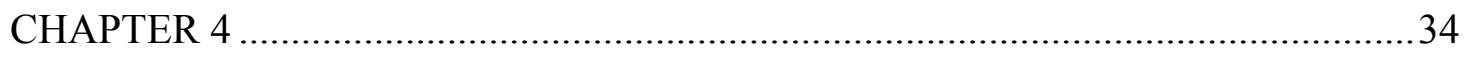

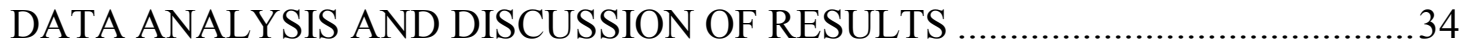

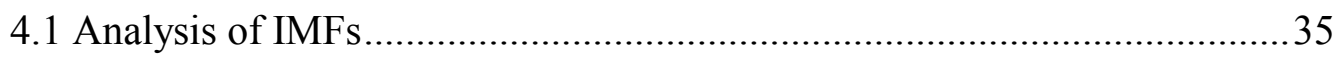

4.2 Energy-Gradient Based Reconstruction:...........................................50

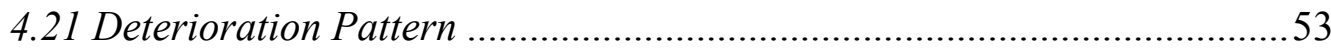

4.22 Localized Profile information ..............................................................54

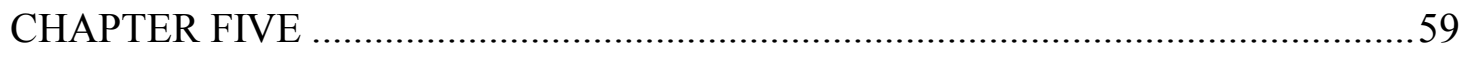

CONCLUSION AND RECOMMENDATIONS ....................................................59

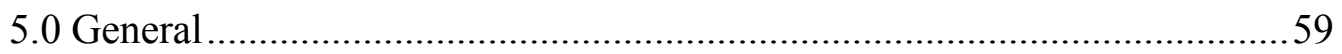

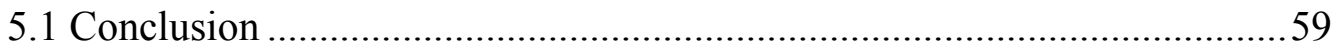

5.2 Recommendations and Future Research ..........................................61

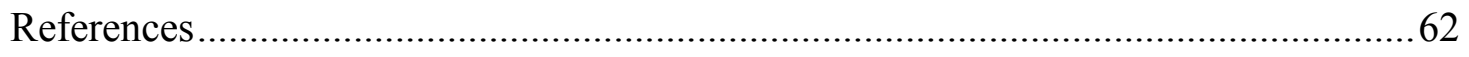

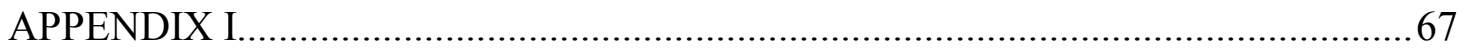

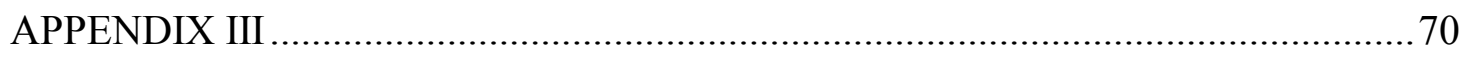

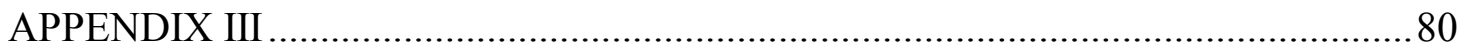




\section{LIST OF FIGURES}

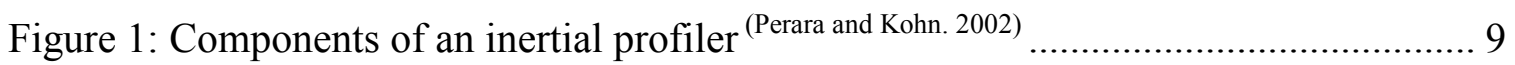

Figure 2: Non-contact lightweight profiler with its non-contact sensor ${ }^{(B u d r a s . ~ 2001)}{ }^{\ldots . . . . . . . . . ~} 10$

Figure 3: McCracken California Style Mechanical Profilograph ${ }^{\text {(Perara and Kohn. 2002) }}{ }^{\text {............ } 11}$

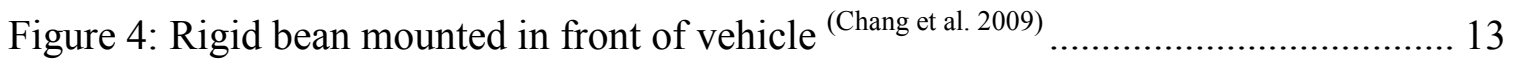

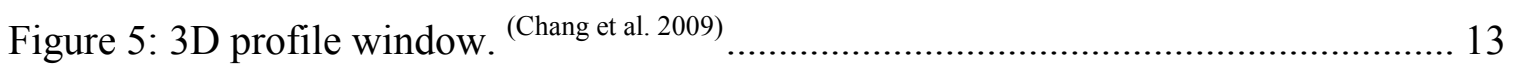

Figure 6: Illustration of moving average computations (Sayers and Karamihas. 2003) $\ldots \ldots \ldots \ldots \ldots \ldots . . . . . .15$

Figure 7: Original road profile and its Power Spectrum............................................ 18

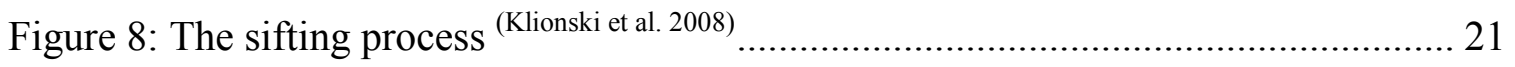

Figure 9: Original road profile selected for the analysis......................................... 35

Figure 10: IMF components resulting from Empirical Mode Decomposition and the

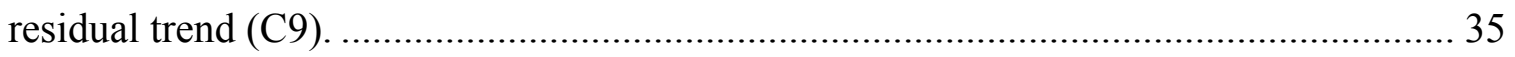

Figure 11: IMF components resulting from Ensemble Empirical Mode Decomposition

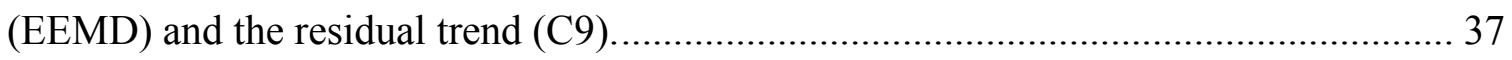

Figure 12: Real IMF components resulting from Complex Empirical Mode

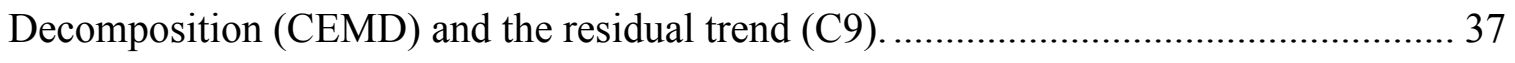

Figure 13: Plot of Instantaneous wavenumber against distance for the highest

wavenumber component IMFs from EMD. 
Figure 14: Plot of Instantaneous wavenumber against distance for the highest wavenumber component IMFs from EEMD. 40

Figure 15: Plot of Instantaneous wavenumber against distance for the highest wavenumber component IMFs from CEMD 41

Figure 16: Correlation coefficients of IMFs with the input signal for the various methodologies (EMD, EEMD, and CEMD). 42

Figure 17: Correlation coefficients of corresponding IMFs for A) CEMD and EEMD.

B). EMD and CEMD. C). EMD and EEMD.

Figure 18: Phase locking values between the first IMF for EMD and EEMD. Reveals phase locking from $40-90 \mathrm{~m}$ and $0-10 \mathrm{~m}$.

Figure 19: Phase locking values between the first IMF for CEMD and EEMD. Reveals phase locking from $40-90 \mathrm{~m}$ and $0-10 \mathrm{~m}$.

Figure 20: Phase locking values between the fourth IMF for EMD and EEMD.

Component is almost completely phase-locked. 46

Figure 21: Phase locking values between the fourth IMF for CEMD and EEMD.

Component is almost completely phase-locked.

Figure 22: Energy-Distance-Frequency plot for IMFs 1-3 from EMD.

Figure 23: Energy-Distance-Frequency plot for IMFs 4-6 from EMD............................. 48

Figure 24:Energy-Distance-Frequency plot for IMFs 1-3 from EEMD. .......................... 48

Figure 25: Energy-Distance-Frequency plot for IMFs 4-6 from EMD............................ 49

Figure 26: Energy-Distance-Frequency plot for IMFs 1-3 from CEMD. 49 
Figure 27: Energy-Distance-Frequency plot for IMFs 4-6 from CEMD.

Figure 28: Plot of mean energy $\left(\mathrm{x} 10^{7}\right)$ against mode number

Figure 29: Reconstructed signal for the deterioration trend analysis............................... 54

Figure 30: Marginal Spectrum of the reconstructed signal showing energy of dominant

wavenumbers

Figure 31: Reconstructed signal for localized profile information 56

Figure 32: Marginal Spectrum of the reconstructed signal showing energy of dominant wavenumbers

Figure 33: Energy-distance-frequency (below) diagram for a selected IMF (above) ....... 58 


\section{LIST OF TABLES}

Table 1: Illustrating the number of IMFs mixing a particular range of wavenumbers from the

EMD.

Table 2: Illustrating the number of IMFs mixing a particular range of wavenumbers from the

EEMD. 38

Table 3: Illustrating the number of IMFs mixing a particular range of wavenumbers from the

CEMD 


\begin{abstract}
Pavement profile analysis is a major component in pavement infrastructure management decision making for maintenance and rehabilitation. This paper takes an in-depth look at pavement profile characterization and evaluation; taking into account the inherent nature of road profile data i.e. non-stationary and nonGaussian. Although there have been several studies aimed at the analysis and characterization of pavement profile, the bulk have been limited to applying relatively conventional signal processing techniques, such as the Fourier analysis. Using this approach, only the average condition of the local conditions can be represented; most transient and changing signals will not be handled well due to the averaging effect of the technique. The Hilbert-Huang transform operates at the scale of every oscillation, an extremely important property for obtaining localized profile information. In this work, the different algorithms of the Hilbert-Huang transform: Empirical Mode Decomposition (EMD), Ensemble Empirical Mode Decomposition (EEMD) and Complex Empirical Mode Decomposition (CEMD) have been discussed and implemented to extract useful information from road profile data. The robustness of the algorithms is compared based on its ability to produce physically meaningful Intrinsic Mode Functions (IMFs) which truly characterize the underlying process. The results show that although all the methodologies yielded similar residual trends, the CEMD produced physically meaningful and trusted IMFs whose information at the various levels of decomposition could be used to extract profile information such as the extent of deterioration and localized roughness information.

Key words: Hilbert-Huang transform; Ensemble Empirical Mode Decomposition; Empirical Mode Decomposition; Complex Empirical Mode Decomposition;
\end{abstract}




\section{Chapter 1}

\section{INTRODUCTION}

\subsection{General}

Pavement profile analysis has become an essential process for assessing and managing paved road surfaces. Road surface unevenness affects ride quality, road safety and vehicle operation cost, so the choice of an effective pavement analysis tool is crucial.

The advent of inertial profilometers which have on-board high speed data acquisition systems, capable of obtaining measurements in the kilohertz range produces huge volumes of data. Intelligent post-processing and handling is needed to extract useful information from real data (Depont and Scott 1999). Currently, profile analysts have adopted various spectral and non-spectral methods to reduce and extract useful information from the voluminous data acquired.

Citing an OCDE (Organisation de Coopération et de Développement Économique) publication (OCDE 1984, Dore et al 2002), report that for the same travelling speed, different wavelengths have various effects on road users. For example, for a $90 \mathrm{~km} / \mathrm{h}$ speed, short wavelengths ranging from $1.25 \mathrm{~m}$ to $2.5 \mathrm{~m}$ (about $20 \mathrm{~Hz}$ to $10 \mathrm{~Hz}$ ) generate excessive motions in vehicle axles and tires, while longer wavelengths ranging from about $8.3 \mathrm{~m}$ to $25 \mathrm{~m}$ (about $3 \mathrm{~Hz}$ to $1 \mathrm{~Hz}$ ) affect the parts of the vehicle above the suspension, causing discomfort to vehicle occupants. Additionally, short wavelengths of $1 \mathrm{~m}$ to $3 \mathrm{~m}$ are associated with degradation of the surface or base layer, while longer wavelengths of about $10 \mathrm{~m}$ are associated with 
subgrade deformation. The Hilbert Huang Transform (HHT) offers the profile analyst the advantage of knowing exactly where these high- and low-frequency events occur along the road, which could inform the maintenance decisions in the pavement management operations.

The International Organization for Standardization employs Fourier analysis to transform the pavement profile data into frequency domain by calculating the power spectral density function (using Fast Fourier Transform) of the profile data. By integrating the Power Spectral density within a specific frequency range, the energy contribution from that frequency range can be obtained and used to categorize the pavement (Depont and Scott 1999). Such analyses, however, can only give information in the frequency or time domain, but not simultaneously. Also, most of the processes used for analysis only provide the average condition of the pavement profile. As a result, they are limited in estimating localized features which sometimes lead to misleading roughness index estimate (a typical example is when a hazardous localized spot appears on a smooth road). The HHT is able to give information on instantaneous frequency and energy content in the time domain without assuming a priori basis functions. The result is better spectral resolution, both in the frequency and time domains. The resulting IMFs are related to physical features in the profile. This implies that filtering and frequency band analysis can be easily performed, helpful for the identification of bumps and dips in our pavement profile (Huang et al 2003).

The motivation for this study arises from the need to properly characterize road profiles based on frequency and energy of the profile. Such a characterization could indicate the response of vehicles traveling on a particular section of road 
regarding severity of vehicle vibrations that are a result of the roughness of the road surface.

The internal structure of a Digitized profile from a flexible pavement is analyzed by using the different HHT algorithms to decompose the original profile into a set of Intrinsic Mode Functions (IMFs). Based on the results, a criterion is defined for selecting non-spurious IMFs and the findings are extended to analyze the profile. An energy-gradient, level-by-level classification process is used to reconstruct the IMFs to extract physically meaningful features from the profile.

The results show that although all the methodologies yielded similar residual trends, the CEMD produced physically meaningful and trusted IMFs whose information at the various levels of decomposition could be used to extract profile information such as the extent of deterioration and localized roughness information.

\subsection{Research Objectives}

The objective of this research is outlined as follows:

- To characterize road profiles based on frequency and energy of the profile. This will enable us to estimate the severity of vibrations that are as a result of the road profile undulation.

- To analyze and compare the performance of the different Hilbert Huang algorithms based on the information revealed by IMFs.

- To design a classification and reconstruction process for extracting physically meaningful information for pavement profile analysis. 


\subsection{Statement of the Problem}

The serviceability or riding quality, safety and vehicle operation cost of a road pavement is a major indicator of its service performance. Due to increases in traffic volume, size and speed of vehicles, social requirements for road surface conditions have become strict all over the world. In order to maintain paved road surfaces efficiently and effectively, it has become essential to conduct research in evaluating the unevenness of road profiles. This area has attracted much attention from researchers and catapulted a number of research studies worldwide. Although the state of the art offers convenient numerical indices for pavement evaluation and management planning, they suffer from a common limitation which is listed below:

- Inability of current methods to detect the presence of localized irregularities such as depressions, humps, settlements and potholes in the profile analyzed. This calls for a methodology which is capable of identifying short changes in the amplitude of elevation as well as the location where the particular irregularity occurs.

- The need to properly characterize road profiles based on frequency and energy of the profile. Such a characterization could indicate the response of vehicles traveling on a particular section of road regarding severity of vehicle vibrations that are a result of the roughness of the road surface.

- The rising need for extracting useful information for network pavement management and pavement maintenance operations.

Valuable information mentioned above is lost and this consequently

affects and distorts pavement maintenance management and planning goals. An appropriate pavement evaluation and analysis tool should provide both numerical 
indices and the above mentioned information to aid pavement maintenance management. The Hilbert Huang Transform is capable of providing further insight into the characteristics of the profile of interest. The procedure is able to identify the characteristics of a pavement roughness profile in both the frequency, amplitude and distance domains.

\subsection{Study Approach}

A review of current methodologies is done to help us understand the state of the art and also appreciate reasons for improving upon these methods. The different Hilbert Huang algorithms will be thoroughly reviewed and used to extract meaningful information from pavement profile. The robustness of the Hilbert Huang methodologies is tested through its ability to suppress the effect of intermittent noise and produce an accurate estimation of residual trend. Essentially, the development of this thesis involves the following steps:

1. General introduction to the use of the Hilbert Huang Transform for pavement profile analysis; highlighting the objectives and the gaps created by traditional profile analysis methods.

2. The second step is an overview of profile analysis and how it has evolved over time. An in-depth review of the state of the art (this includes the methods as well as the equipments used for profile analysis) is also presented and used as a background for introducing the prowess of the HHT.

3. Step three gives a comprehensive review of the methodology employed by the different Hilbert Huang algorithms, i.e. the EMD, EEMD and CEMD. The 
underlying assumptions and possible challenges of these assumptions are also clearly explained.

4. The interpretation and analysis of our results is the next step in this organization. Initially the performance of the algorithms is based on visual analysis; then, we introduce correlation and phase locking methods to buttress our conclusions. Finally, an energy gradient classification process is proposed for grouping and combining the IMFs to extract information for profile analysis.

5. The last step covers the major conclusions made in the study and the future direction of this study. 


\section{Chapter 2}

\section{BACKGROUND AND LITERATURE REVIEW}

\subsection{General}

A profile is a two-dimensional slice of the road surface, taken along an imaginary line (Sayers and Karamihas 2003). The lateral view or line through a profile gives an indication of the superelevation and crown of the road design, plus rutting and other distresses. Longitudinal profiles will show the design grade, roughness, and texture (Sayers and Karamihas 2003). Our primary focus in this paper will be on longitudinal profiles. Pavement profile data is usually obtained by using devices called profilers to pick up surface elevation values along a section of the pavement. The different types of profilers (rod and level, dip stick and inertial profilers) have its own well-defined way of representing the true profile.

Profile measurements are carried out on pavements on regular basis either to monitor the condition of a road network for pavement management, or to evaluate the ride quality of newly constructed or overlaid pavements. Over the years, different equipments have been used for profiling: Generally categorized into 3 groups:

1. Response type road roughness measuring systems (RTRRMs)

2. Profilographs

3. Inertial profilers

RTRRMS devices were traditionally used to measure road roughness. They are housed in automobiles or standardized trailers. Automobile housed systems accumulate the vertical movement of the rear axle of the automobile with respect to 
frame; while the trailer mounted system accumulate the movement of the trailer with respect to frame. The vertical displacements are accumulated and divided by the distance to report roughness in terms of inches per mile. The main difficulty with the RTRRMs is that measurements obtained from such devices are influenced by vehicle dynamic properties such as suspension, tire conditions, tire pressure and vehicle weight. Owing to the above factors the response collected by the device will vary with time and also with the type of vehicle. This has led to replacement of RTRRMs by inertial profilers.

Advances in laser technology have expedited the development of Inertial profilers used to collect profile data on pavements not only at highway speeds but also it requires a certain speed even to function. It does not work well at speeds less than $15 \mathrm{~km} / \mathrm{hr}$ (Sayers and Karamihas 2003). High speed inertial profilometers are normally used at the network level to measure roughness for pavement management purposes as opposed to project level for profilographs and light weight profilometers (Chang et al 2009). The profilers measure surface elevations of pavements using non contact sensors by combining the following three ingredients (Dore et al 2002):

1) A reference elevation;

2) A height relative to the reference; and

3) Longitudinal distance.

A schematic diagram of an inertial profiler is shown in Figure 1.

All the inertial profilers presently available are based on the inertial profiler design originally developed by Sprangler and Kelly at the GMR Laboratories (Spangler and Kelly 1966). The principal components of any inertial profiler are height sensor, accelerometers, distance measuring system, and computer hardware and 
software for computation of the road profile (Perara and Kohn 2002). The height sensors record the distance between the pavement surface and the vehicle. The accelerometers located on top of the height sensors measure the vertical acceleration of the vehicle. The distance measuring system measures the distance traveled from a starting reference point. Using the data obtained from the height sensor, accelerometer and distance measuring system, computer algorithms compute the profile of a pavement surface.

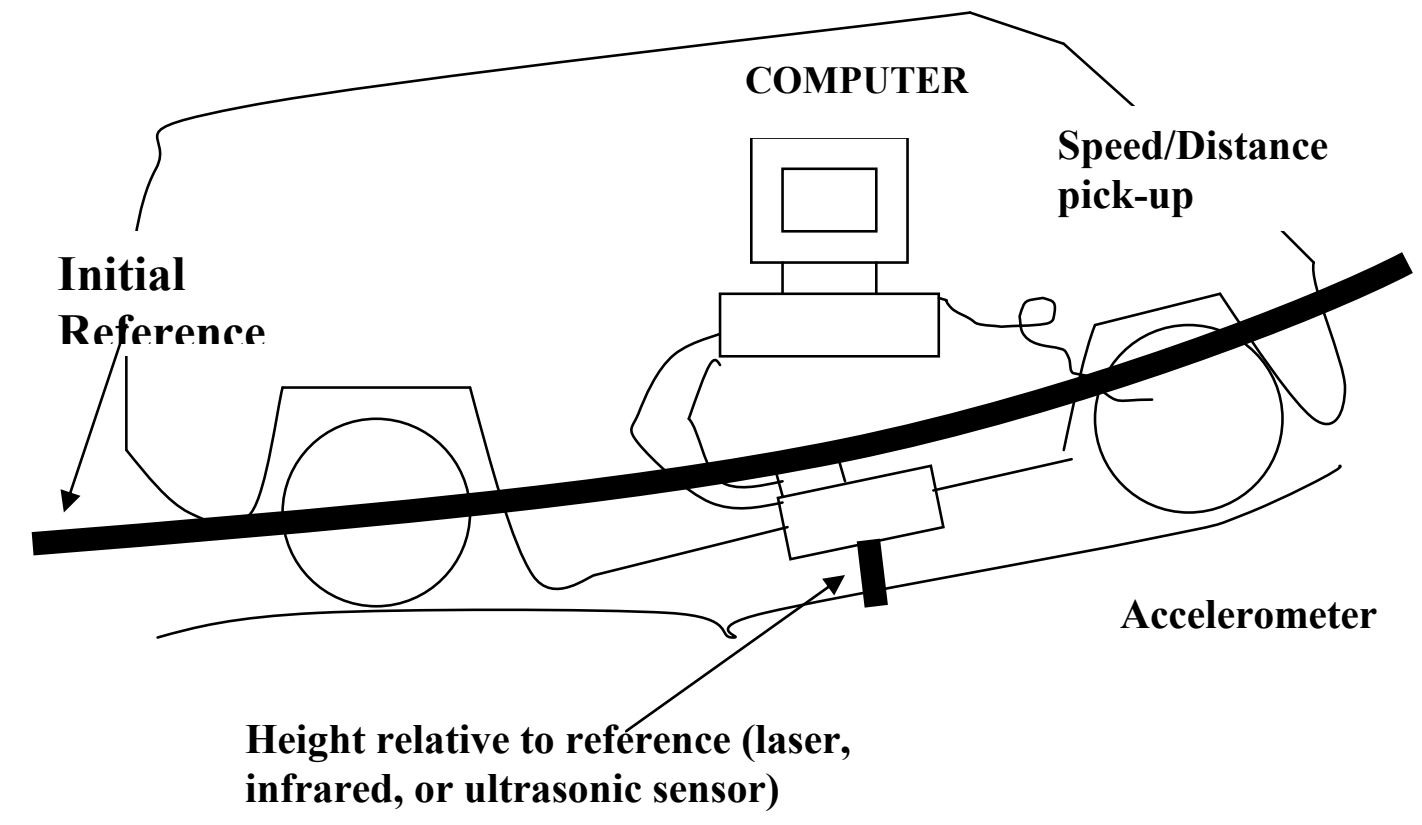

Figure 1: Components of an inertial profiler (Perara and Kohn 2002)

Light weight non-contact inertial profilers are also available for construction quality control and quality acceptance purposes. It is known for its timely 
and accurate measurements although it requires operators to perform repetitive, tedious and time-consuming procedures (Chang et al 2009). The profiling system in light weight profilers is same as that in inertial profilers and is installed in a light vehicle such as a golfcart or an all terrain vehicle.

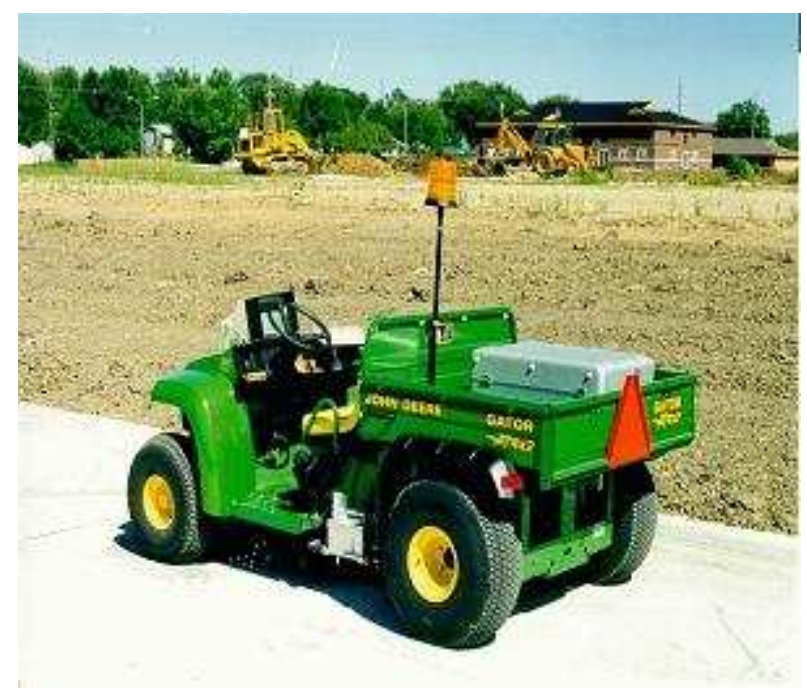

Figure 2: Non-contact lightweight profiler with its non-contact sensor

(Budras 2001)

These profilers have very low operating speeds ranging from $8(13 \mathrm{~km})$ to 25 miles $(40 \mathrm{~km})$ per hour, which makes it impractical for high speed, large road network data collection (Budras 2001).

The profilograph is used to measure the longitudinal profile of a concrete pavement. Its principal use has been for the construction control of pavements. The profilographs are relatively inexpensive, simple to operate and maintain, and provides 
a trace of surface users can easily understand (Budras 2001). In the early to mid 20th century, a typical model of profilographs consisted of a rigid beam with support wheels at either end and a centre wheel. The support wheels at the ends establish a datum from which the deviations of the centre wheel can be evaluated (Perara and Kohn 2002). A strip chart recorder or a computer records the deviations of the centre wheel with respect to the end wheels. The data recorded in the case of a strip chart is analyzed manually to obtain PI and defect locations. However a scanner based system is available called, ProScan that permits the automated analysis of manual profilographs. A profilograph with an onboard computer, records and analyses data in the field immediately after the tests, thus eliminating the need for manual analysis of data (Perara and Kohn 2002). Two of such types of profilographs are still in massive use as of today: the California profilographs and the Ames profilograph.

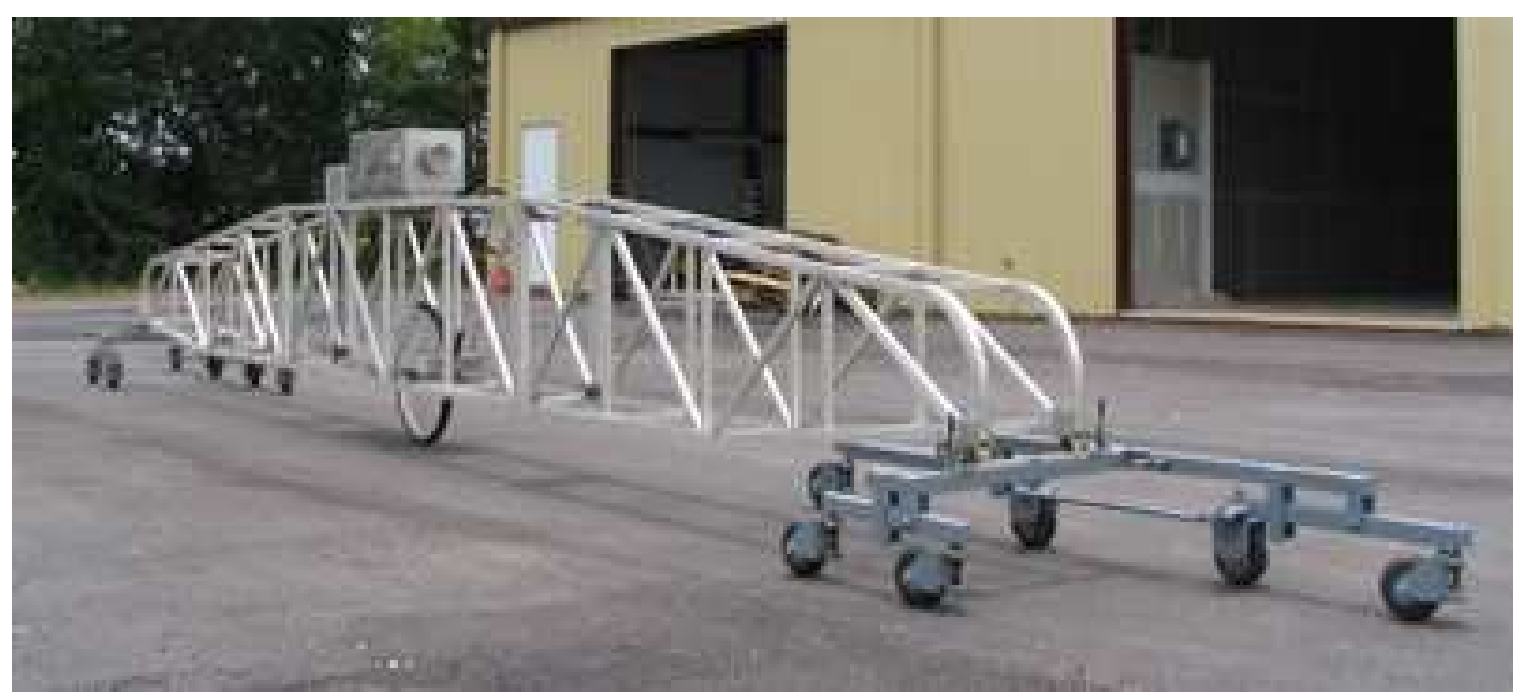

Figure 3: McCracken California Style Mechanical Profilograph (Perara and Kohn 2002) 
All these profilographs essentially work on the same principle but differ in support wheel configuration or frame support. Figure 4 shows a California profilograph with a beam length of $7.65 \mathrm{~m}(25 \mathrm{ft})$. There are two support wheel systems at either end of the profilograph. Clearly from the figure 3 , it can not be used for high speed network pavement smoothness data collection. They are used for project level pavement management purposes.

These days, there are some advanced, high precision and high speed profilographs which are capable of picking the transverse and longitudinal profile and combining it into a one 3D profile as shown in figure 5 . An Odometer mounted on the wheel of a vehicle combined with the inertial system keeps precise track of the vehicle movement. Combined with the lasers a full 3D profile is calculated. Together with Texture sensors and GPS a full overview of the surface condition is presented in the software. Post processing software synchronizes the $3 \mathrm{D}$ profile with the other measurements made in the same vehicle based on the high precision Odometer. 


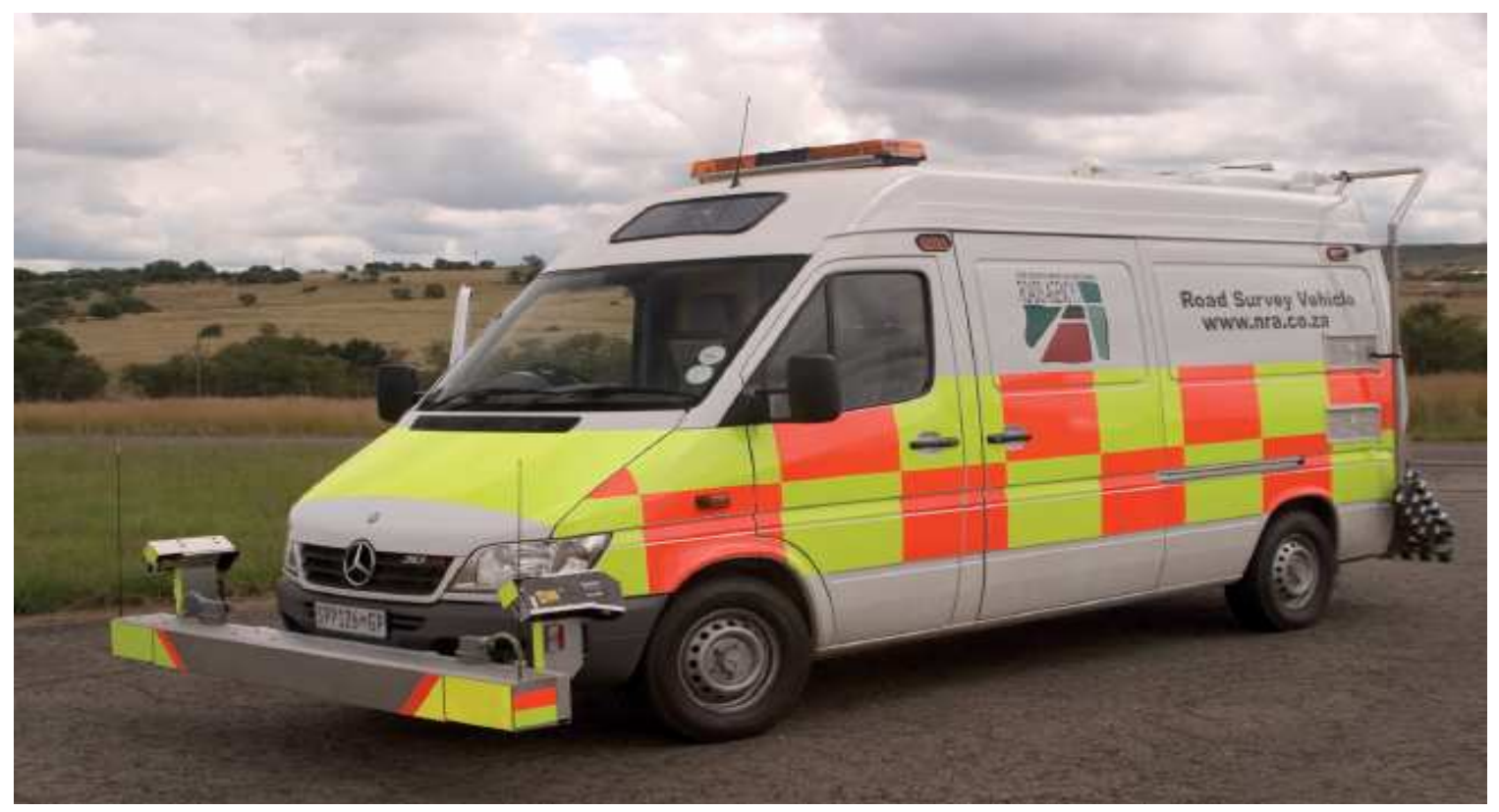

Figure 4: Rigid beam mounted in front of vehicle (Chang et al 2009)

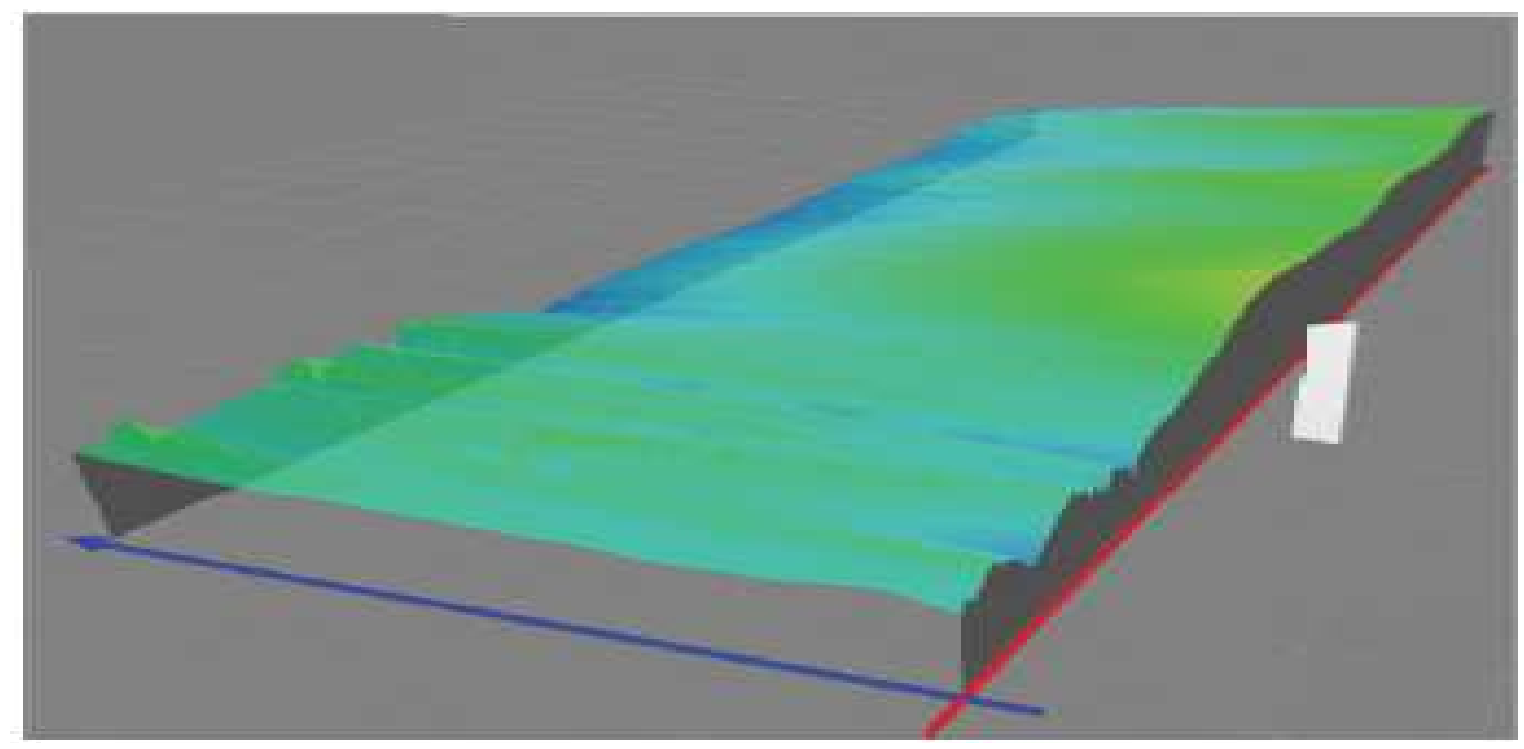

Figure 5: 3D profile window. (Chang et al 2009) 
Analyzing profile data provides the civil engineer, managers and planners with much information that can be used to evaluate and manage the condition of road networks. Such analyses of road profile data falls into the category of signal processing. An important class of signal processing is filtering. It is almost always mandatory to filter profile data in order to:

1. improve the quality of measurements by eliminating unwanted 'noise' from the data and

2. extract useful information of interest from profile data.

3. view different types of profile feature.

A common and simple method for description and analysis of profile data is band-pass filtering. In this method, a moving filter window is created and applied to the profile in short sections; it replaces each profile point with the average of several adjacent points.

This implies that low frequencies will be passed well while attenuating the amplitudes of high frequencies. The effect of short wavelengths and spiking amplitudes are reduced. A smoothed profile or a low-pass filter is calculated as:

$$
P_{f l}(i)=\frac{1}{N} \sum_{j=1-\frac{B}{2 \Delta x}}^{i+\frac{B}{2 \Delta x}} P(j)
$$

Where $P_{f l}$ is the smoothed profile.

$\mathrm{B}$ is the base length of the moving average

$\mathrm{N}$ is the number of samples included in the summation (little book on profiling) and $\Delta x$ is the window length. 


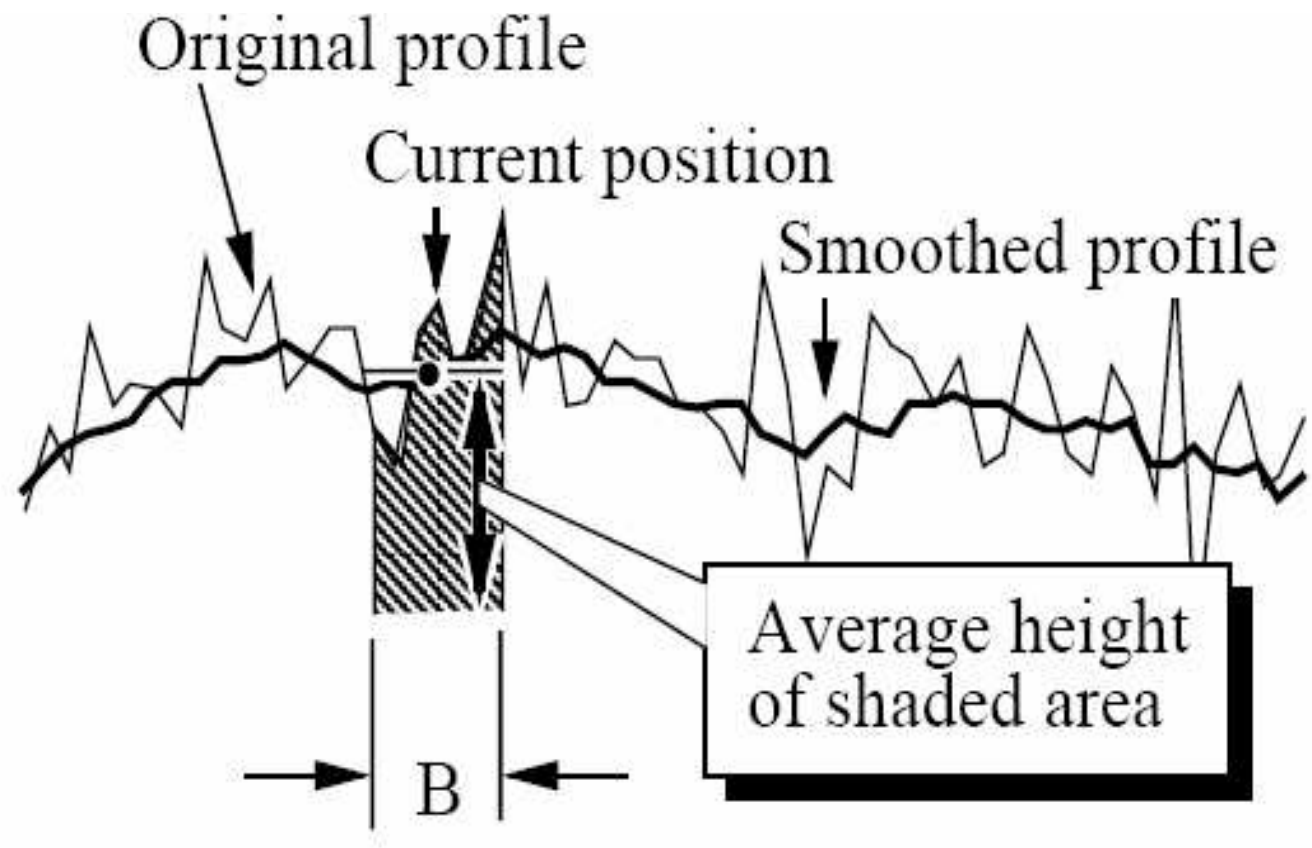

Figure 6: Illustration of moving average computations (Sayers and

Karamihas 2003).

By removing the smoothed profile from the original profile we obtain a high-pass filter designated as:

$$
P_{f H}(i)=P(i)-\frac{1}{N} \sum_{j=1-\frac{B}{2 \Delta x}}^{i+\frac{B}{2 \Delta x}} P(j)
$$

The high-pass filter produces an anti-smoothing effect by passing all high frequencies of the profile well but reducing the amplitude of frequencies lower than the threshold frequency. This helps to reduce the effects of long wavelengths in the profile.

The main issue with the moving average filter has to do with its assumption of arbitrary cut-offs (thresholds). When it is applied to a profile that 
includes large bumps and dips, it will reduce or amplify the extent of the localized feature, consequently resulting in spurious energy values for that feature. In this sense the moving average takes away some of the required information as a result of smoothing.

Currently, both spectral and non-spectral methods have been designed to reduce (filter) and extract useful information from the pavement profile data acquired.

\subsection{Non-Spectral Techniques}

A widely accepted non-spectral method is the International Roughness Index (IRI) estimation. The IRI provides an average profile condition rating. It is calculated by accumulating the absolute values of the suspension deflection and dividing by the distance travelled. The IRI shows significant response to wavelengths between $1.1 \mathrm{~m}$ and $44 \mathrm{~m}$. Due to this response to longer wavelengths, the IRI is

normally calculated over pavement sections greater that $200 \mathrm{~m}$ in length. Experiments have shown that repeatability of IRI values over short lengths is very bad. For this reason it is not prudent to use IRI in monitoring changes in profile over short lengths. (Depont and Scott 1999). IRI does not provide a comprehensive and detailed description of road profiles, although it is the most widely used descriptor of pavement roughness levels. Rather, it gives an indication of the ride quality relative to road roughness in the form of an aggregated index. The IRI also does not provide frequency information; it only accumulates the response of the dynamic system against its position. Furthermore, the IRI does not consider the non-stationary nature of road profile data, which has been established by Bruscella et al. According to Rouillard et al 1996, some of the main drawbacks of the IRI are as follows: 
(1) It cannot be used to simulate vibration levels or road profiles.

(2) Since the response of the quarter car model is not typical of real vehicles, such as a sedan or a steel-suspended truck, the IRI cannot represent the dynamic behaviour of the real vehicles.

(3) The constant window-width of the smoothing functions often attenuates significant short wavelength events and thus alters the character of the process.

\subsection{Spectral Techniques}

The adoption of spectral techniques for analyzing pavement profiles is very necessary as most profiles generally have many of the statistical properties of random signals (Marcondes 1991).

The International Organization for Standardization employs Fourier analysis to transform the pavement profile data into frequency domain by calculating the power spectral density function (using Fast Fourier Transform) of the profile data. By integrating the Power Spectral Density within a specific frequency range, the energy contribution from that frequency range can be obtained and used to categorize the pavement (Depont and Scott 1999). A simpler approach is to use linear regression to fit a straight line to the PSD plot. The magnitude of the slope which is normally called the waviness can be extracted. Also the value of the line at wavenumber of 0.1 cycles/m (comparative density), can be extracted (Depont and Scott 1999). The strength of this spectral method over the IRI is its ability to know which wavenumber (frequency) is contributing to roughness. A typical Power Spectral Density plot is 
shown in figure 7. Such analyses, however, can only give information in the frequency or time domain, but not simultaneously. Also, most of the processes used for analysis only provide the average condition (means effects of transients cannot be separated) of the pavement profile. As a result, they are limited in estimating localized features which sometimes lead to misleading roughness index estimate (a typical example is when a hazardous localized spot appears on a smooth road).
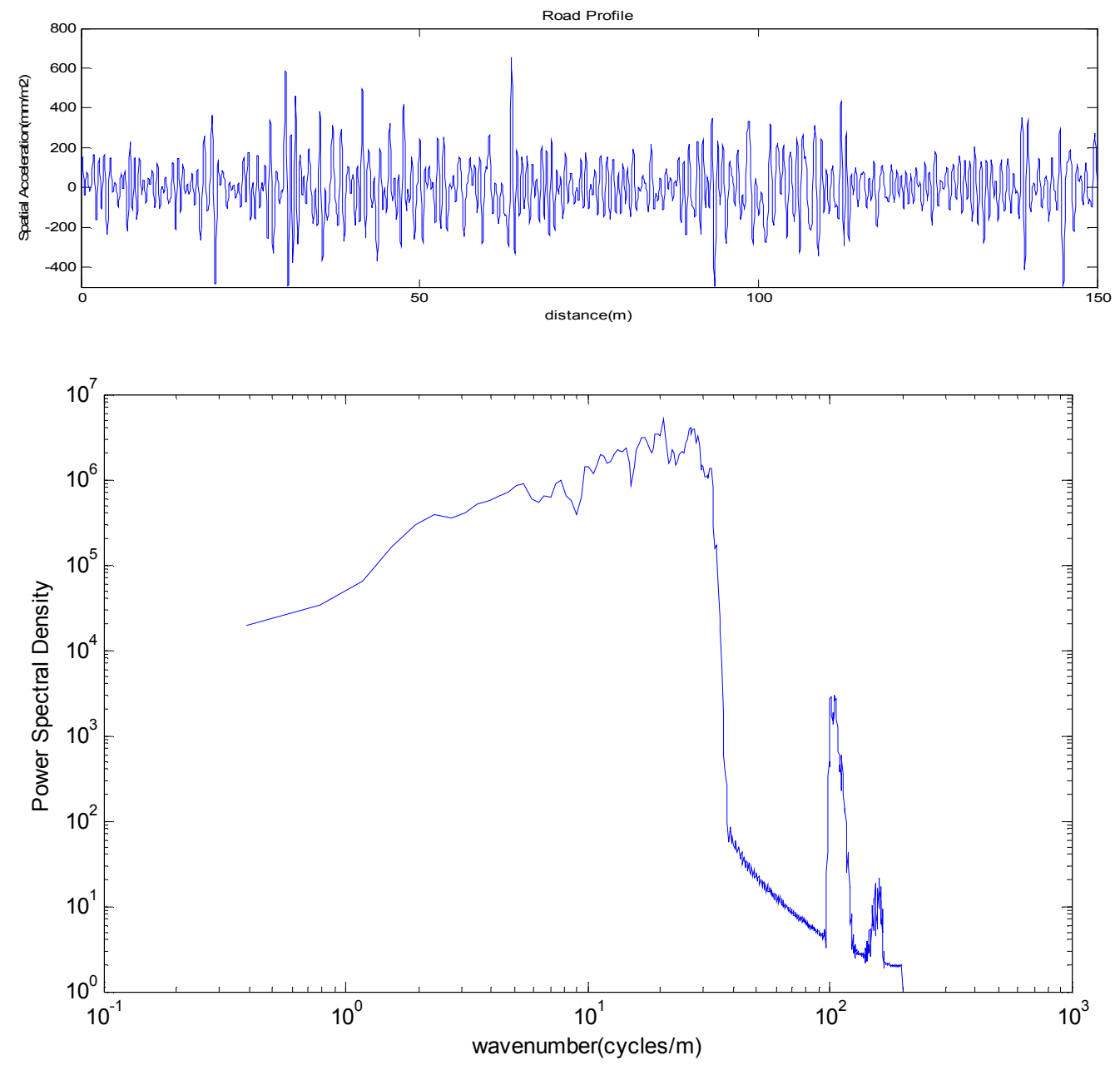

Figure 7: Original road profile and its Power Spectrum. 
The wavelet is also another spectral method which decomposes data into segments which are consequently transformed into distance frequency plane rather than just frequency or distance domain. It is able to avoid the Heisenberg uncertainty by its adjustable-width basis functions called wavelets. These wavelets are capable of extracting low and high frequency components of a signal by stretching or compressing its width. A wavelet is zero mean and has a finite duration. This property encourages localized analysis that gives both good frequency and temporal resolutions. By summing up components level by level, the contribution at each level enables us to find locations where the magnitude of the contribution to the signal is significantly higher, than the reference value. Although the wavelet appears similar to the Fourier analysis, there are three basic differences:

1. The basis functions in the wavelet are not limited to the sinusoidal waves, but rather are chosen by the user to address various problems of time-frequency resolution;

2. While the Fourier Transform uses a single analysis window width, the wavelet principally uses short windows at high frequencies and longer windows at low frequencies: and

3. When the scale dilation factor, in the wavelet is changed, the duration and the bandwidth of the basis function are both simultaneously changed, yet the original shape of the mother wavelet is retained. Nonetheless, wavelet analysis is also limited by the fundamental uncertainty principle, in which both time and frequency cannot simultaneously be resolved with the same precision. Moreover, the analysis 
results depend on the choice of mother wavelet which is arbitrary and may not be optimal for the time-series being analyzed.

A desirable feature of the wavelet method is the uniform resolution of scales; however, this feature can be disadvantageous when there is a poor resolution since it will affect all the scales. Additional disadvantages include spectral leakage and it being non-adaptive (only the basic wavelet chosen is used in the whole analysis) (Ayenu-Prah and Attoh Okine 2008).

The disadvantages of the Fourier and wavelet transforms prompted the development of a new spectral analysis method called the HHT. 


\section{Chapter 3}

\section{DEVELOPMENT OF THE METHODOLOGY}

\subsection{The Hilbert-Huang Transform}

The Hilbert Huang Transform (HHT) remains a very powerful tool for data analysis due to its ability to process non-stationary. HHT consists of two key components namely: Empirical Mode Decomposition (EMD) and Hilbert Spectral Analysis. A summary of the process is provided below (Huang et al 2003, Huang et al 1998 and Klionski et al 2008).

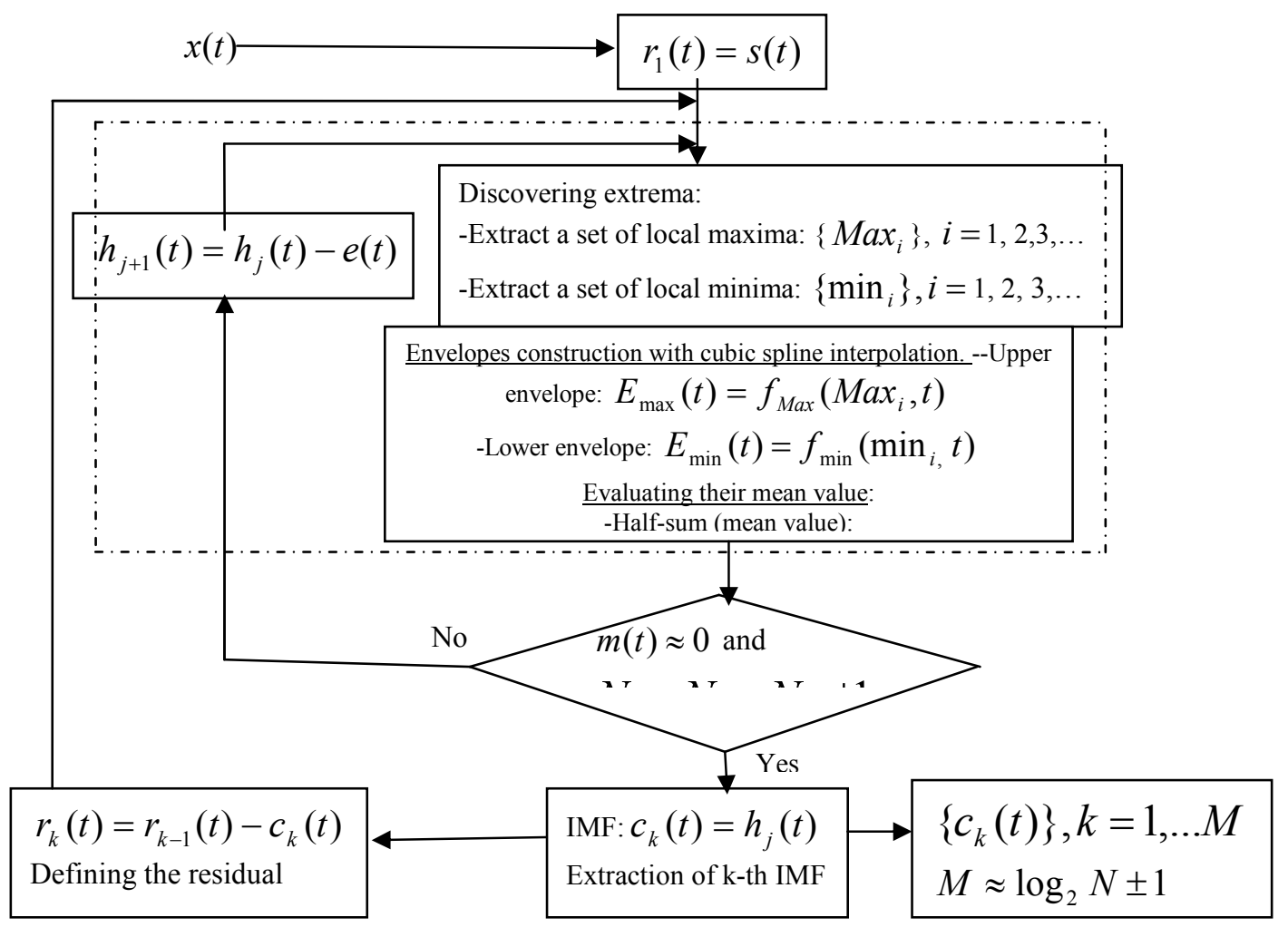

Figure 8: The sifting process (Klionski et al 2008) 
The first component of the HHT is the EMD. The essence of the EMD is to decompose different trends or fluctuations contained in the complicated signal gradually through their characteristic scales to obtain a series of data sequence with different intrinsic timescales, i.e., the IMFs. This decomposition helps better understand the internal structure of the signal and the components involved (Klionski et al 2008).The EMD assumes that, at any given time there may be many different coexisting modes of oscillation, each superimposed on the others. Therefore, empirical decomposition should provide finite modes that are independent of the others. The EMD algorithm is highly efficient and adaptive as it preserves the non-stationary and nonlinear characteristics in the IMFs (basis functions) through its local wave analysis property (Zhou et al 2009). These IMFs mainly possess two important properties (Huang et al 1998, Klionski et al 2008):

a) The number of extrema and the number of zero crossings must either equal or differ by no more than one in the whole data set.

$$
N_{\text {max }}+N_{\text {min }}=N_{\text {zero }} \pm 1
$$

Where $N_{\max }$ is the total number of maxima, $N_{\min }$ is the total number of minima, and $N_{\text {zero }}$ is the total number of zero crossings. This condition is similar to the traditional narrow band requirement for a stationary Gaussian process. It assumes that the signal has at least two extrema-one maximum and one minimum; if the data were totally devoid of extrema but contained only inflection points, then it can be differentiated once or more times to reveal the extrema.

b) At any point the mean $m(t)$ of the envelope defined by the local maxima, and the envelope defined by the local minima, is zero.

$$
\frac{E_{\max }(t)+E_{\min }(t)}{2} \approx 0,
$$


Where $E_{\max }(t)$ is the envelope of the local maxima and $E_{\min }(t)$ is the envelope of the local minima by spline interpolation.

An IMF is similar to the Fourier-based simple harmonic function, except that it is more general, having both amplitude and frequency modulations. The empirical modes are extracted from a complicated data by the following process:

First, identify all the local extrema (maxima- $\{\mathrm{Max}\}$ and minima$\{\mathrm{Min}\})$ of the input signal $X(t)$ where $\mathrm{t}$ belongs to $[1, \ldots, \mathrm{N}]$. Then connect all the local maxima by a cubic spline to produce the upper envelope $E_{\max }(t)$.

$$
E_{\max }(t)=f_{\max }\left(\operatorname{Max}_{i}, t\right),
$$

The procedure is repeated for the local minima to produce the lower envelope $E_{\min }(t)$.

$$
E_{\text {min }}(t)=f_{\min }\left(\operatorname{Min}_{i}, t\right),
$$

The upper and lower envelopes should encompass all the data between them. This implies:

$$
\operatorname{Min}(t) \leq X(t) \leq \operatorname{Max}(t)
$$

Their mean is designated as:

$$
m_{1}(t)=\frac{E_{\min }(t)+E_{\max }(t)}{2}
$$

and the difference between the data $X(t)$ and $m_{1}(t)$ is the first component $h_{1}$ :

$$
X(t)-m_{1}(t)=h_{1}(t)
$$

Ideally, $h_{1}(t)$ should be an IMF. Errors might be introduced due to the spline fitting process. This calls for repetition of previous process, normally referred to as a "sifting process". In the subsequent sifting processes, $h_{1}(t)$ is treated as the data and a new mean is computed:

$$
h_{11}(t)=h_{1}(t)-m_{11}(t)
$$

Here, $m_{11}$ is the mean of the upper and lower envelopes for $h_{1}$. 
After repeating the sifting process up to $\mathrm{k}$ times, $h_{1 k}$ becomes an IMF:

$$
h_{1 k}(t)=h_{1(k-1)}(t)-m_{1 k}(t)
$$

So, $h_{1 k}$ becomes the first IMF. To reduce the complexity of the following equations, we replace $h_{1 k}$ with $C_{j}, j=1, \ldots n . C_{1}$ contains the finest-scale or the shortest-period component of the signal. $C_{1}$ is removed from the rest of the data to obtain the residue:

$$
r_{1}(t)=X(t)-C_{1}(t)
$$

The residue $r_{1}$, is treated as new data since it contains information on longer-period components, and subjected to the same sifting process as described above. The sifting procedure is repeated for all subsequent $r_{i}$ functions as:

$$
r_{j-1}-C_{j}=r_{j} ; \mathrm{j}=2,3 \ldots \mathrm{n}
$$

The whole EMD process is terminated when results meet the following criteria:

1) Either the component $C_{n}$ or the residue $r_{n}$ becomes so small that it is less than a predetermined value of consequence, or;

2) The residue $r_{n}$ becomes a monotonic function, from which no more IMF can be extracted.

The original data are thus the sum of the IMF components plus the final residue:

$$
X(t)=\sum_{j=1}^{n} C_{j}(t)+r_{n}(t)
$$

$C_{j}$ is the $\mathrm{jth}$ IMF, and $\mathrm{n}$ is the number of sifted IMF in the above equation. $r_{n}$ represents either the mean trend or a constant. Over-sifting can smooth the amplitudes of IMFs, rendering them physically less meaningful. Over-sifting is avoided by the following relations:

1. By limiting the size of the sum of the difference (SD), computed from two consecutive sifting results as: 


$$
S D=\frac{\sum_{t=0}^{T}\left|h_{k-1}(t)-h_{k}(t)\right|^{2}}{\sum h_{k-1}^{2}(t)}
$$

2. By thresholding

$$
\begin{aligned}
& \frac{2\left|E_{\max }(t)+E_{\min }(t)\right|}{\left|E_{\text {max }}(t)-E_{\text {min }}(t)\right|} \leq \Theta_{1} \text {, for }(100-\alpha) \% \text { of samples } \\
& \frac{2\left|E_{\text {max }}(t)+E_{\text {min }}(t)\right|}{\left|E_{\max }(t)-E_{\text {min }}(t)\right|} \leq \Theta_{2} \text {, for } \alpha \% \text { of samples. }
\end{aligned}
$$

The thresholds $\Theta_{1}, \Theta_{2}, \alpha$ are usually expected to be equal to the following values:

$$
\Theta_{1}=0.05, \Theta_{2}=10 \Theta_{1}=0.5, \alpha=5 \%
$$

Based on numerous experimental studies, the total number of IMFs is evaluated by the following formula:

$$
\text { IMFS }=\log _{2} N \pm 1
$$

The second and final step after the decomposition is based on Hilbert transform. To transform from a time-space data to a time-frequency data, we apply the Hilbert Transform to each IMF, which yields instantaneous frequency and amplitude.

For given data, $\mathrm{X}(\mathrm{t})$, the Hilbert transform, $\mathrm{Y}(\mathrm{t})$, is defined as:

$$
Y(t)=\frac{1}{\pi} P \int_{-\infty}^{\infty} \frac{X(\tau)}{t-\tau} d \tau
$$

P represents the Cauchy principal value. With this definition, $X(t)$ and $Y(t)$ can be combined to form the analytical signal $\mathrm{Z}(\mathrm{t})$, given by:

$$
Z(t)=X(t)+i Y(t)=a(t) e^{i \theta(t)}
$$

$\mathrm{a}(\mathrm{t})$ and $\theta(\mathrm{t})$ represent the amplitude and phase, respectively:

$$
\begin{aligned}
& a(t)=\sqrt{X^{2}+Y^{2}} \\
& \theta(t)=\tan ^{-1} \frac{Y}{X}
\end{aligned}
$$


Instantaneous frequency can be defined as:

$$
\omega(t)=\frac{d \theta(t)}{d t}
$$

After applying the Hilbert transform, each IMF is represented as:

$$
C_{j}=\operatorname{Re}\left[a_{j}(t) e^{i \int w_{j}(t) d t}\right]
$$

This implies that the original equation will be expressed as:

$$
X(t)=\operatorname{Re} \sum_{j=1}^{n} a_{j}(t) e^{i \int w_{j}(t) d t}
$$

Equation 22 is written in terms of amplitude and instantaneous frequency associated with each component as functions of time. This differs from the time-independent amplitude and phase in the Fourier series representation of the following:

$$
X(t)=\operatorname{Re} \sum_{j=1}^{\infty} a_{j} e^{i w_{j} t}
$$

Where $a_{j}$ and wj are constants. A comparison of the two representations in equations 23 and 24 suggests that the Hilbert transform of the IMF can be considered as a generalized Fourier expansion. The time-dependent amplitude and instantaneous frequency in equation 23 can be represented in a three-dimensional plot, where the amplitude is contoured on the time-frequency plane. This frequency time distribution of the amplitude is designated as the Hilbert amplitude spectrum $H(\omega, t)$, or simply Hilbert spectrum. With the Hilbert spectrum defined, we can define the marginal spectrum $h(\omega)$ as:

$$
h(\omega)=\int_{0}^{T} H(\omega, t) d t
$$

The Hilbert spectrum is convenient for discovering hidden amplitude and frequency modulations in signals and finding out the domains of energy and frequency concentration (Klionski et al 2008). The marginal spectrum offers a measure of total 
amplitude (or energy) contribution from each frequency value. It represents the cumulated amplitude over the entire data span in a probabilistic sense.

An essential implementation of the EMD has been realized by using its perfect reconstruction property for detrending and denoising signals (Fladrin et al 2004). The process of denoising is based on classifying IMFs as signal-like or noiselike based on their energy levels. Usually, an energy threshold is chosen experimentally for the particular class of signals needed. The sum of all signals below the chosen threshold becomes the denoised signal. This procedure for denoising a signal is attractive due to its simplicity yet, its assumption about the nature of the noise embedded in a signal is problematic. Noise is intermittent, that is, it can be present in some areas and absent in other parts of the signal (Zhou et al 2009). This defect leads to the introduction of a noise assisted data analysis algorithm called the EEMD.

\subsection{Ensemble Empirical Mode Decomposition}

A major drawback of the EMD described above is a result called mode mixing. Real data almost always contain some intermittencies or random noise. As a result, an effective decomposition method should be sensitive to added noise of small but finite amplitude. Mode mixing results when the decomposition method is unable to collate signals with similar frequencies into each IMF. As a result, different modes of oscillations reside in each IMF, making IMFs lose physical meaning or falsely represent the physical processes in a mode. This drawback resulted in the introduction of the EEMD, a noise assisted data analysis method. The EEMD is aimed at automatically identifying and removing the relevant IMFs that contribute to the intermittent noise and background intensity. Thus the component representing 
fundamental frequency is obtained and the desired information, such as phase distribution, can be easily extracted via Hilbert transform (Zhou et al 2009). Below is a summary of the EEMD process described by Wu and Huang 2005.

The EEMD employs multiple applications of EMD; the significance of the introduction of white noise should be noted as such. White noise contains all possible frequencies of vibration or sound, as such, when added to the target data, it provides a uniform distribution over the entire time-frequency space. This provides a reference frame for the signals of comparative scale to collate in one IMF, thus eliminating "mode mixing". The ensemble mean of corresponding IMFs is used to cancel out white noise leaving only the true signal. The EEMD is described as follows (Yaguo et al 2009).

1. Initialize the number of ensemble $\mathrm{M}$, the amplitude of the added white noise, and $m=1$.

2. Perform the mth trial on the signal added white noise

a. Add white noise series with the given amplitude to the investigated signal

$$
x_{m}(t)=x(t)+\eta_{m}(t)
$$

Where $\eta_{m}(t)$ indicates the mth added white noise series, and $x_{m}(t)$ represents the noise-added signal of the mth trial.

b. Decompose the noise-added signal $x_{m}(t)$ into IMFs I imfs $c_{i, m}(i=1,2, \ldots, I)$ using the EMD method described in the previous section, where $c_{i, m}$ is the $\mathrm{i}^{\text {th }} \mathrm{IMF}$ of the $\mathrm{m}^{\text {th }}$ trial, and $\mathrm{I}$ is the number of IMFs.

c. If $m<M$ then go to step (a) with $m=m+1$. Repeat steps (a) and (b) again and again, but with different white noise series each time. 
3. Obtain an ensemble mean of the corresponding IMFs $C_{i}$ of the decomposition

$$
\begin{aligned}
& \overline{C_{i}(t)}=\frac{1}{M} \sum_{m=1}^{M} C_{i, m-1}(t)+r_{m}(t), \\
& i=1,2, \ldots ., I, m=1,2, \ldots ., M,
\end{aligned}
$$

4. Report the mean $\overline{C_{i}(t)}$ of the IMFs as the final IMFs.

The effect of the added white noise should decrease following the wellestablished statistical rule:

$$
\begin{aligned}
& \varepsilon_{n}=\frac{\varepsilon}{\sqrt{N}} \\
& \ln \varepsilon_{n}+\frac{\varepsilon}{2} \ln N=0
\end{aligned}
$$

Here, $\varepsilon$ is the amplitude of the added noise and $\varepsilon_{n}$ is the final standard deviation of error, which is defined as the difference between the input signal and the corresponding IMFs.

Instantaneous frequency concept is only meaningful for monocomponent signals, which are symmetric about a local mean (Loutridis 2004). Due to this fact, applying the Hilbert transform to the components resulting from EEMD is meaningless, because IMFs may not be symmetric about the local mean. The components are post-processed using the traditional EMD to obtain monocomponent modes, which are symmetric about the local mean.

\subsection{Complex Empirical Mode Decomposition (CEMD)}

The application of the traditional EMD and the EEMD to profile analysis is based on the assumption that a signal's oscillation is univariate. A new extension of 
the EMD to handle and analyze data which are intrinsically bivariate (complex-valued) was introduced by (Rilling et al 2007, Gagarin et al 2004, Tanka and Mandic 2007). The proposed method is based on the assumption that a bivariate signal is equal to fast rotations superimposed on slower rotations.

It has long been recognized that the use of complex exponential have distinct advantages over operations with real harmonic (and non-harmonic) functions. Considering a simple harmonic function, there are two ways of representing the real harmonic function with a complex exponential. One is to replace the harmonics by the resultant of two complex vectors rotating in opposite directions.

$$
\begin{aligned}
& \cos w t=\frac{1}{2}\left(e^{j w t}+e^{-j w t}\right) \\
& \sin w t=\frac{1}{2}\left(e^{j w t}-e^{-j w t}\right)
\end{aligned}
$$

The other is to use the real part of a single rotating vector. Hence a real signal of the form

$$
x(t)=a \cos w t+b \sin w t
$$

can be replaced by a complex time function of the form:

$$
x_{\text {complex }}(t)=x(t)+j \sigma(t)=(a-j b) e^{j w t}
$$

which is formed by adding to the real signal $x(t)$ an imaginary signal $j \sigma(t)$. The imaginary signal $\sigma(t)$ is the Hilbert transform of the original signal. The function $j \sigma(t)$ has a simple significance. It represents the signal in quadrature (ninety degree out of phase with the in phase component) to $x(t)$ which, added to it, transforms the oscillating signal into a rotating vector. If $x(t)$ is not a simple harmonic function, the process by which $x_{\text {complex }}(t)$ has been obtained can easily be generalized (Gabor.1946). 
The advantages of a complex representation for real signal stems from a natural separation of amplitude information from angle (which includes both phase and frequency) information. Normally, in real signals these are blended in a way which is confusing to visual analysis. It should be remembered throughout this section that the complex representation in itself is not a signal processing technique wherein the information contained in the signal is altered in some way. Rather, it is a representation of exactly the original signal information which may be more amenable to visual analysis than the original signal itself (Farnbach 1975).

1. Initialization: $\mathrm{k}=1$, Project the complex-valued signal $x(t)$. onto a predetermined number of projection directions $\varphi_{n}$. The number of directions should satisfy:

$$
\varphi_{n}=2 n \pi / N, n \in[1, N] .
$$

The projected signal is now represented as;

$$
p_{\varphi_{n}}=\operatorname{Re}\left(e^{-j \varphi_{n}} x_{k}(t)\right), \quad n \in[1, N] \text {. }
$$

2. Extract the maxima of $p_{\varphi_{n}}(t)$ and calculate the corresponding envelopes $e_{\varphi_{n}}(t)$ by spline interpolation in all projection directions.

3. Compute the mean of the envelopes in the different projection directions

$$
m_{k}(t)=\frac{1}{N} \sum_{n=1}^{N} e_{\varphi_{n}}(t)
$$

4. Subtract the mean $m_{k}(t)$ from the original signal $x(t)$. to obtain $h_{k, i}(t)$

$$
h_{k, i}(t)=x_{k}(t)-m_{k}(t)
$$

Let $x(t)=h_{k, i}(t)$ and repeat steps (1) through (3). Over-sifting is avoided in the same way as the EMD, by limiting the size of the sum of the difference (SD), computed from two consecutive sifting results as: 


$$
S D=\frac{\sum_{t=0}^{T}\left|h_{k-1}(t)-h_{k}(t)\right|^{2}}{\sum h_{k-1}^{2}(t)}
$$

If $h_{k, i}(t)$ meets the stopping criteria then it becomes the first complex IMF.

5. Record the obtained IMF. Let $x_{k}(t)=x(t), k=k+1$.

6. Repeat steps 1-5 until K IMFs are calculated.

\subsection{Phase Locking}

Recently, phase locking analysis has become an increasingly popular method of investigating synchronization between time series especially in the area of EEG (Electroencephalogram) data analysis (Sweeney and Nasuto 2007). It was originally proposed in an attempt to quantify neural synchrony observed between pairs of electrodes in EEG data. It is defined as a near constant phase difference persisting over a time period (Sweeney and Nasuto 2007). As already mentioned, phase information alone can be obtained in various ways. These include using the Fourier Transform (FT), the Wavelet Transform (WT), and the Hilbert Transform (HT). The phase information extracted in this work employs the Hilbert transform. As stated earlier, the pavement profile consists of a superposition of individual component signals. Therefore an analytical signal representation of the profile, although could provide a natural phase definition, it is not useful when analyzing phase synchrony among the individual components of the multi-component signal. It becomes useful only when the data is decomposed into narrow bandwidths using filters. This procedure has been gracefully performed by the Hilbert Huang algorithm in this work. A phase locking index can therefore be accurately defined for corresponding IMFs of the different HHT algorithms. 
The phase locking index (PL) varies from 0 when there is no phase locking to 1 when the phases are completely locked. When corresponding IMFs have high phase locking index ( $\mathrm{PL}>0.8)$, it means that the signals are coherent and may have similar information or behavior. Low PL values indicate lack of coherence. The phase locking index between two signals is calculated as:

$$
P L(f, t)=\left|1 / \delta \int_{t-\delta / 2}^{t+\delta / 2} e^{i\left(\theta_{y}(f, t)-\theta_{x}(f, t)\right.} d t\right|
$$

Where $\operatorname{PL}(f, t)$ is the phase lock index, is the time window over which the PL's are calculated.

$\theta_{y}$ and $\theta_{x}$ are the phases of corresponding IMFs $x$ and $y$ at each frequency $f$ and time $t$. 


\section{Chapter 4}

\section{DATA ANALYSIS AND DISCUSSION OF RESULTS}

With over 1500 meters $(\mathrm{m})$ of pavement profile data measured at a $0.05 \mathrm{~m}$ sampling period, a section $150 \mathrm{~m}$ in length was selected for the analysis presented in this thesis. This implies that the analysis presented is based on one-dimensional (longitudinal) profile data information. It should also be noted however, that road surface elevation data has been transformed into spatial acceleration (Bruscella et al 1999). The spatial acceleration used is the second derivative of the elevation profile. This magnifies amplitudes of shorter wavelength components, eliminating any linear trend (Ayenu-Prah and Attoh Okine 2008). It is also an indication of the vertical acceleration of a vehicle traveling along the pavement. Also, by employing the Hilbert transform, real signals now are represented in the complex domain to enable us to use the CEMD. Profile analysis was carried out using MATLAB ${ }^{\circledR}$, the EEMD and Complex EMD codes were prepared by Wu et al.

Profile data (figure 9) from the selected pavement type has been decomposed using EMD, EEMD and CEMD. The results are shown in figures 10, 11 and 12 . 


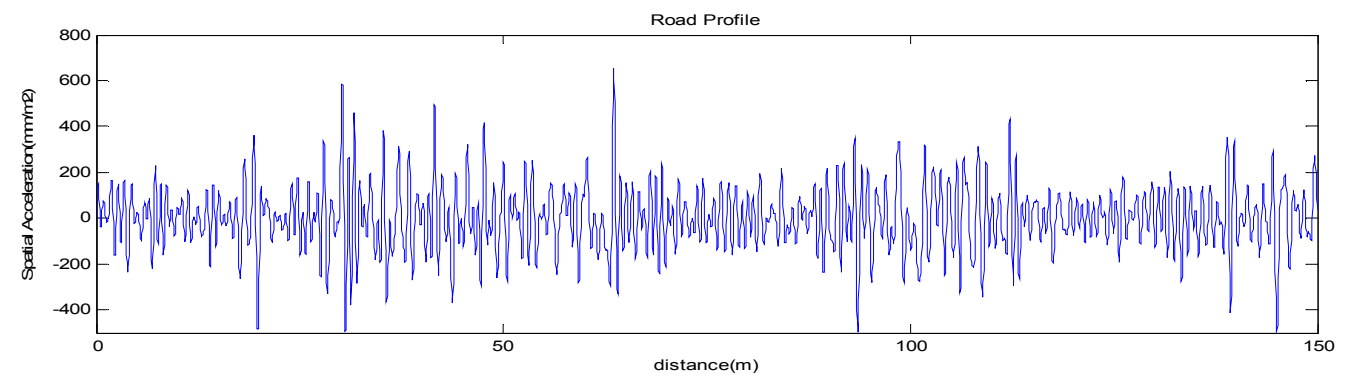

Figure 9: Original road profile selected for the analysis.
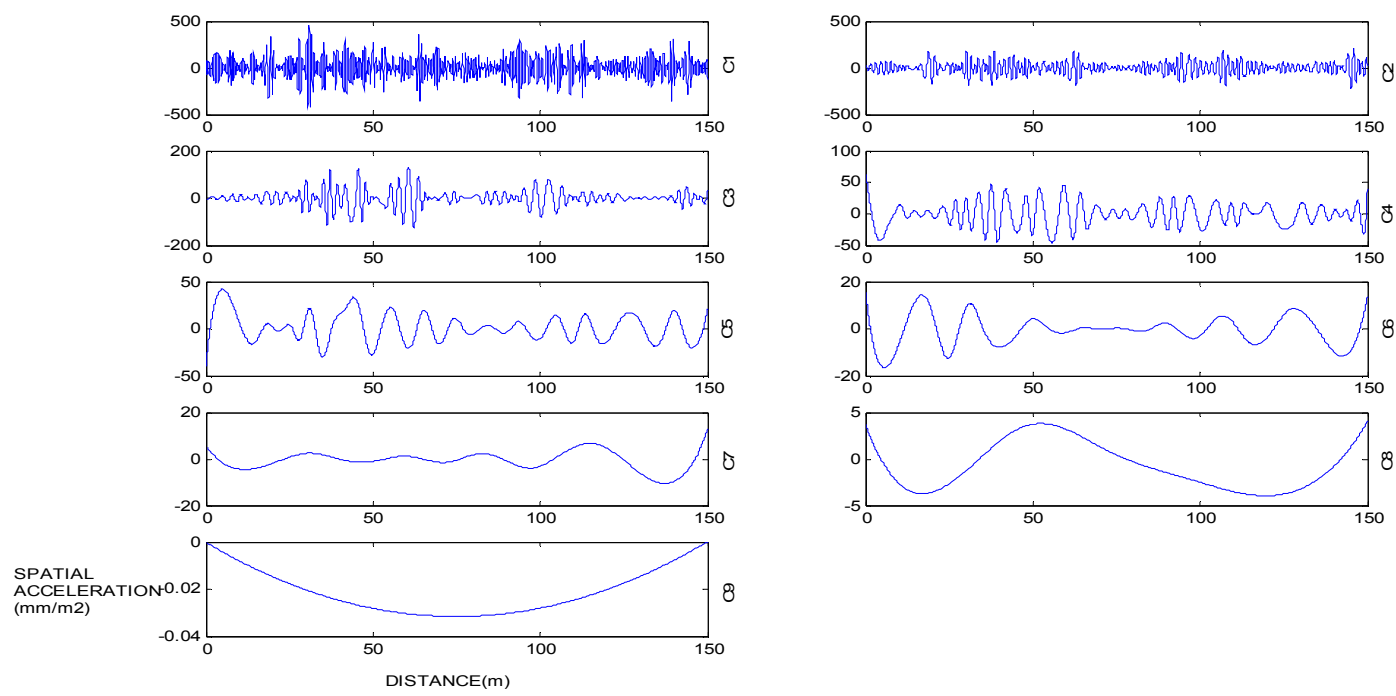

Figure 10: IMF components resulting from Empirical Mode Decomposition and the residual trend (C9).

\subsection{Analysis of IMFs}

The finest modes $(\mathrm{C} 1)$ usually contain the noise in the profile. If the signal is denoised well, the next finest scale (C2) could be considered as guidance into analyzing the texture of profiles; distinct peaks in the original profile still remain but it is smoothened out. From figures 10, 11\&12, all the IMFs have very low amplitude and 
frequency variations between $0-20 \mathrm{~m}, 60-90 \mathrm{~m}$ and $120-140 \mathrm{~m}$ as compared to other sections of the profile. These areas are possibly smooth sections of the profile. In sections where different amplitudes and frequencies exist, the credibility of the information presented is highly uncertain. The modes could present spurious information if narrow-band frequencies are mixed.

For this reason, the first objective in this paper will be to analyze which modes have mixed frequencies. The information presented from such modes cannot be trusted for the analysis. Secondly, the IMFs extracted are expected to have a positive correlation with the original signal. Negative or zero correlations also cannot be trusted since they carry information different from what the original signal contains.

Thirdly, two concepts are used to bring to light the kind of information gained or lost as a result of the choice of a particular algorithm. The two basic concepts are employed to achieve this purpose are;

1. Phase-locking and

2. Cross-correlation coefficient.

Cross-correlation coefficient values are highly dependent on the amplitude of the signal. As a result the phase-locking technique is introduced in the analysis to help separate phase information from amplitude information; in so doing, the analysis is able to focus on the temporal information between the signals. 

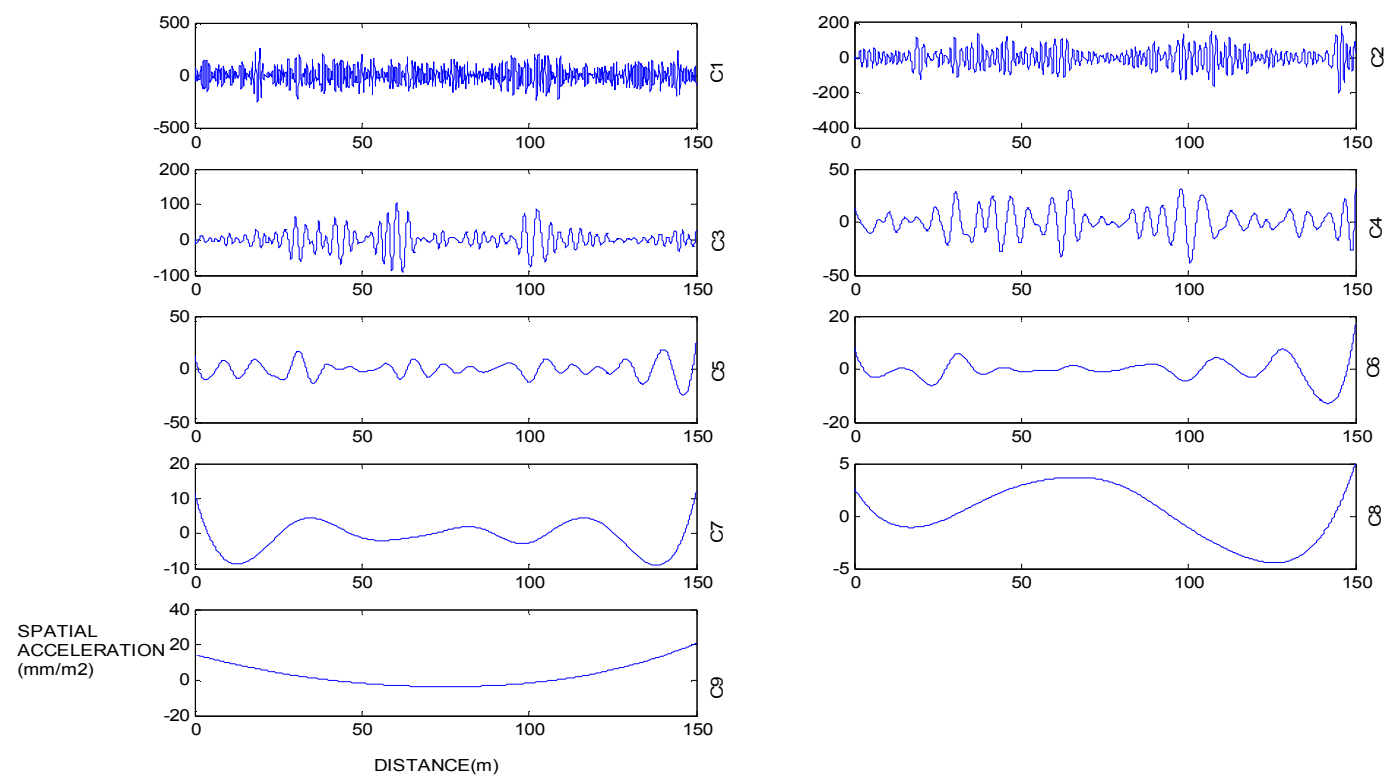

Figure 11: IMF components resulting from Ensemble Empirical Mode Decomposition (EEMD) and the residual trend (C9).
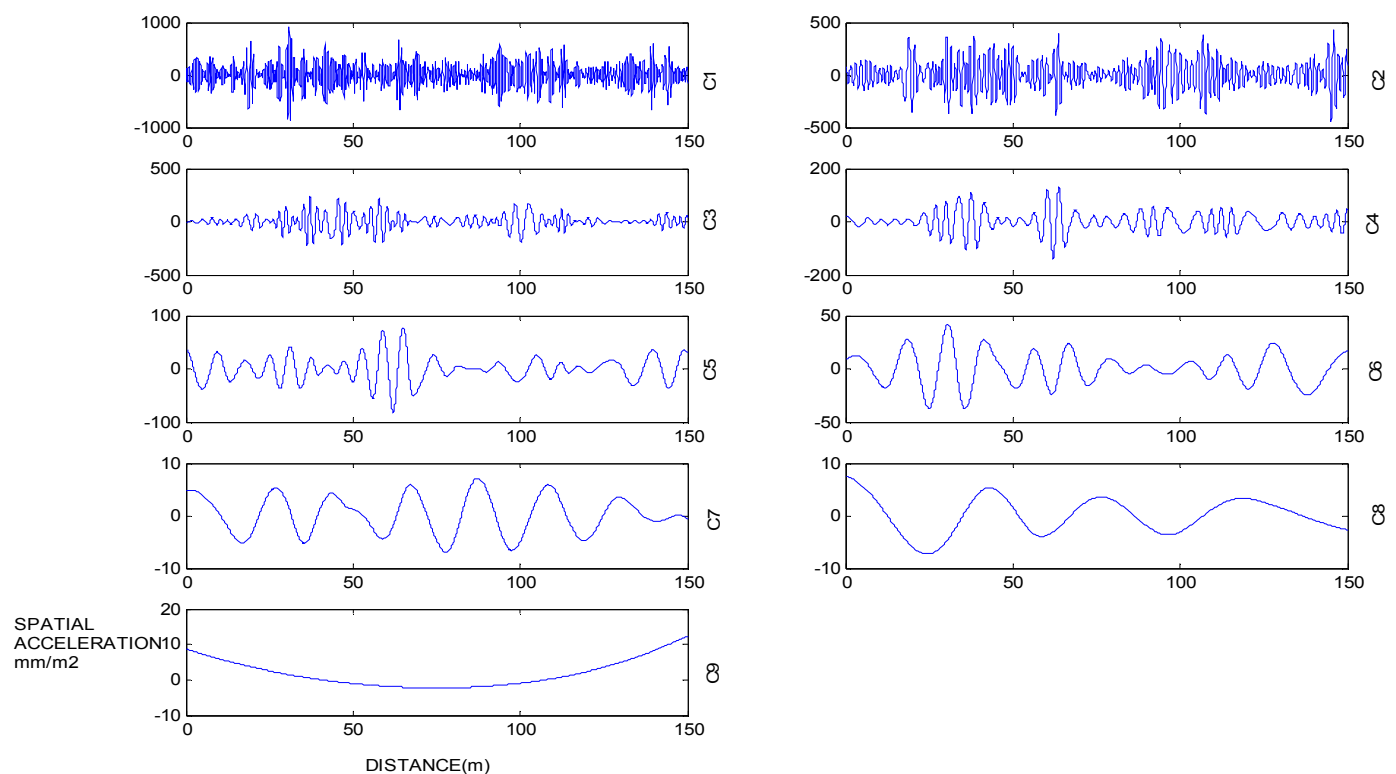

Figure 12: Real IMF components resulting from Complex Empirical Mode Decomposition (CEMD) and the residual trend (C9). 
Tables 1-3 illustrate the percentage of some selected ranges of wavenumber (frequency) in the different modes. Although the EEMD performs better than the EMD, both mixes the wavenumber ranges in at least 3 modes. For example in table 1, the highest wavenumber range 1.0-1.5 is distributed among three IMFs, constituting $65 \%$ of the wavenumbers in IMF $1,34 \%$ of the wavenumbers in IMF 2 and $12 \%$ in IMF 3.

Table 1: Illustrating the number of IMFs mixing a particulare range of wavenumbers from the EMD

\begin{tabular}{|c|c|c|c|c|c|c|c|c|c|}
\hline & \multicolumn{9}{|c|}{ IMF Number } \\
\hline \multirow{6}{*}{ 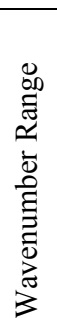 } & & 1 & 2 & 3 & 4 & 5 & 6 & 7 & 8 \\
\hline & $1.0-1.5$ & 0.654 & 0.342 & 0.118 & * & * & * & * & * \\
\hline & $0.5-1.0$ & 0.316 & 0.612 & 0.705 & 0.1319 & * & * & * & * \\
\hline & $0.1-0.5$ & * & * & 0.114 & 0.83 & 0.44 & 0.43 & * & * \\
\hline & $0.05-0.1$ & * & * & * & * & 0.52 & 0.48 & 0.53 & 0.27 \\
\hline & $0-0.05$ & * & * & * & * & * & * & 0.47 & 0.71 \\
\hline
\end{tabular}

Table 2: Illustrating the number of IMFs mixing a particular range of wavenumbers from the EEMD.

\begin{tabular}{|c|c|c|c|c|c|c|c|c|c|}
\hline & \multicolumn{9}{|c|}{ IMF Number } \\
\hline \multirow{6}{*}{ 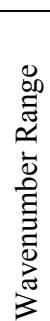 } & & 1 & 2 & 3 & 4 & 5 & 6 & 7 & 8 \\
\hline & $1.0-1.5$ & 0.775 & 0.124 & * & * & * & * & * & * \\
\hline & $0.5-1.0$ & 0.196 & 0.782 & 0.605 & 0.092 & * & * & * & * \\
\hline & $0.1-0.5$ & * & * & 0.346 & 0.902 & 0.34 & * & * & * \\
\hline & $0.05-0.1$ & * & * & * & * & 0.61 & 0.68 & 0.32 & 0.12 \\
\hline & $0-0.05$ & * & * & * & * & * & 0.35 & 0.63 & 0.81 \\
\hline
\end{tabular}

A similar result is obtained in Table 2 for the EEMD although it spreads the frequencies in 2 IMFs. Their inability to collate these narrow bands in 1 or 2 IMFs 
might distort information within these modes. The CEMD clearly improves on the mode mixing problem. Wavenumber range $1.0-1.5$ is uniquely collated into the IMF 1. In cases where the same wavenumber ranges exist in 2 or three different modes, there are huge percentage differences for the CEMD.

Table 3: Illustrating the number of IMFs mixing a particular range of wavenumbers from the CEMD.

\begin{tabular}{|c|c|c|c|c|c|c|c|c|c|}
\hline \multicolumn{10}{|c|}{ IMF Number } \\
\hline \multirow{6}{*}{ 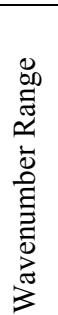 } & & 1 & 2 & 3 & 4 & 5 & 6 & 7 & 8 \\
\hline & $1.0-1.5$ & 0.94 & * & * & * & * & * & * & * \\
\hline & $0.5-1.0$ & * & 0.9725 & 0.5752 & 0.0919 & * & * & * & * \\
\hline & $0.1-0.5$ & * & * & 0.3344 & 0.92 & * & * & * & * \\
\hline & $0.05-0.01$ & * & * & * & * & 0.92 & 0.63 & 0.18 & 0.023 \\
\hline & $0-0.05$ & * & * & * & * & * & 0.30 & 0.77 & 0.917 \\
\hline
\end{tabular}

* - represents modes with very small percentage of the selected range.

A clearer understanding and visualization of the effects of mode mixing on IMFs is demonstrated in the distance-frequency space distribution from figures 13 , 14 and 15. In figure 13, the time frequency distribution for the EMD is gapped (fragmented) and there is no clear separation of scales. Disparate amplitudes and frequencies exist in each IMF, hence, it is not possible to resolve very high and very low amplitudes at the same time; this is why there are gaps. This has several implications with regards to identifying and estimating the ranges of variation of frequencies for analysis. The time frequency distribution for CEMD relative to the EMD and EEMD is much more continuous (no gaps) as seen in figures 14 and 15. This is primarily because IMFs are relatively more continuous and less disparate scales exist in a particular mode. 


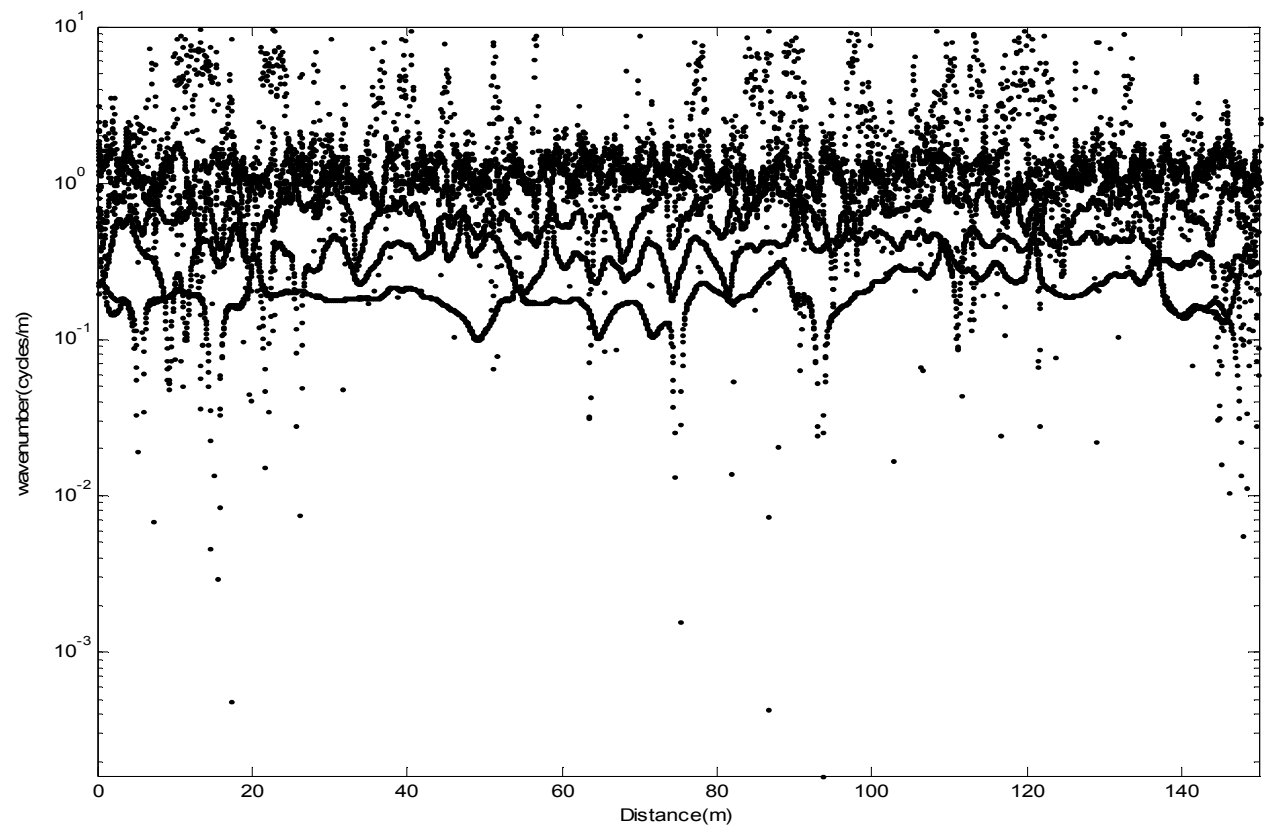

Figure 13: Plot of Instantaneous wavenumber against distance for the highest wavenumber component IMFs from EMD.

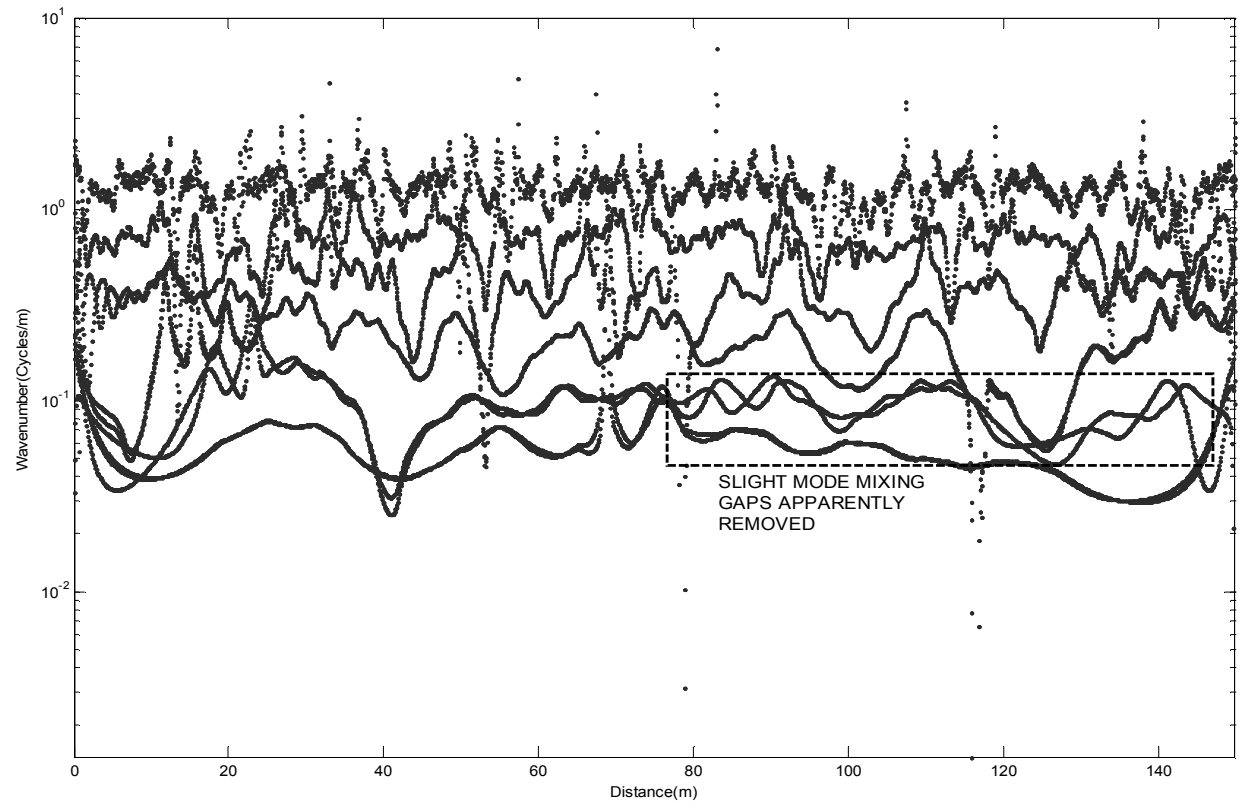

Figure 14: Plot of Instantaneous wavenumber against distance for the highest wavenumber component IMFs from EEMD. 


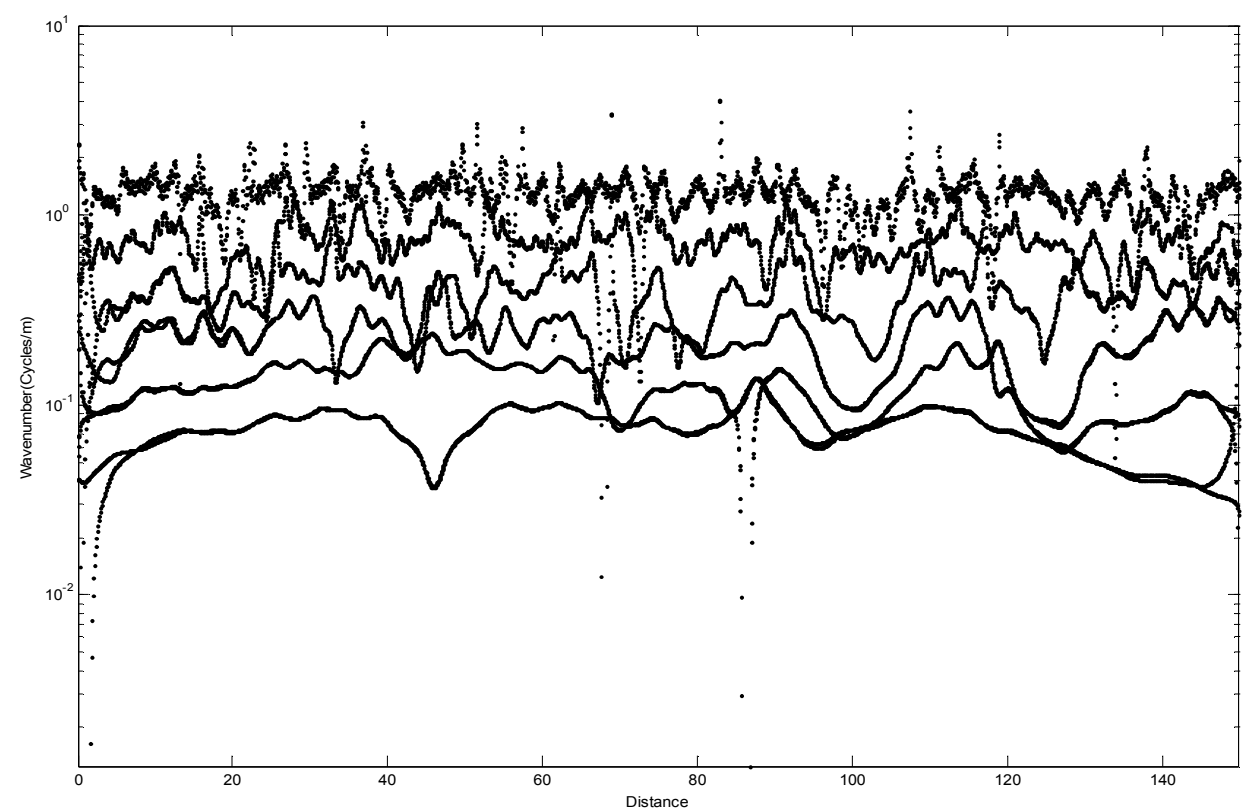

Figure 15: Plot of Instantaneous wavenumber against distance for the highest wavenumber component IMFs from CEMD

In order to investigate the second objective, the IMFs are correlated with the original signal. The result is shown in figure 16. The EEMD and the CEMD follow a similar trend as their correlation values converge with increasing IMF number. There are no non- negative or zero correlations. However, the EMD's correlation values for IMF4 to IMF6 deviate from that of the EEMD and the CEMD. This could be the result of excessive mode mixing at the finest scales. All three methodologies have approximately the same correlation values for the trend (residue). This means that the algorithms could perform creditably well for deterioration trend analysis.

In order to achieve our third objective, corresponding IMFs of the various methodologies are correlated and their correlation values are shown in figure 17 . In a 
perfect sense, corresponding IMFs should contain the same amount of information, but due to limitations of some methodologies, this is not realized. From the figure 17A, we realize very high correlation between IMFs from the CEMD and EEMD.

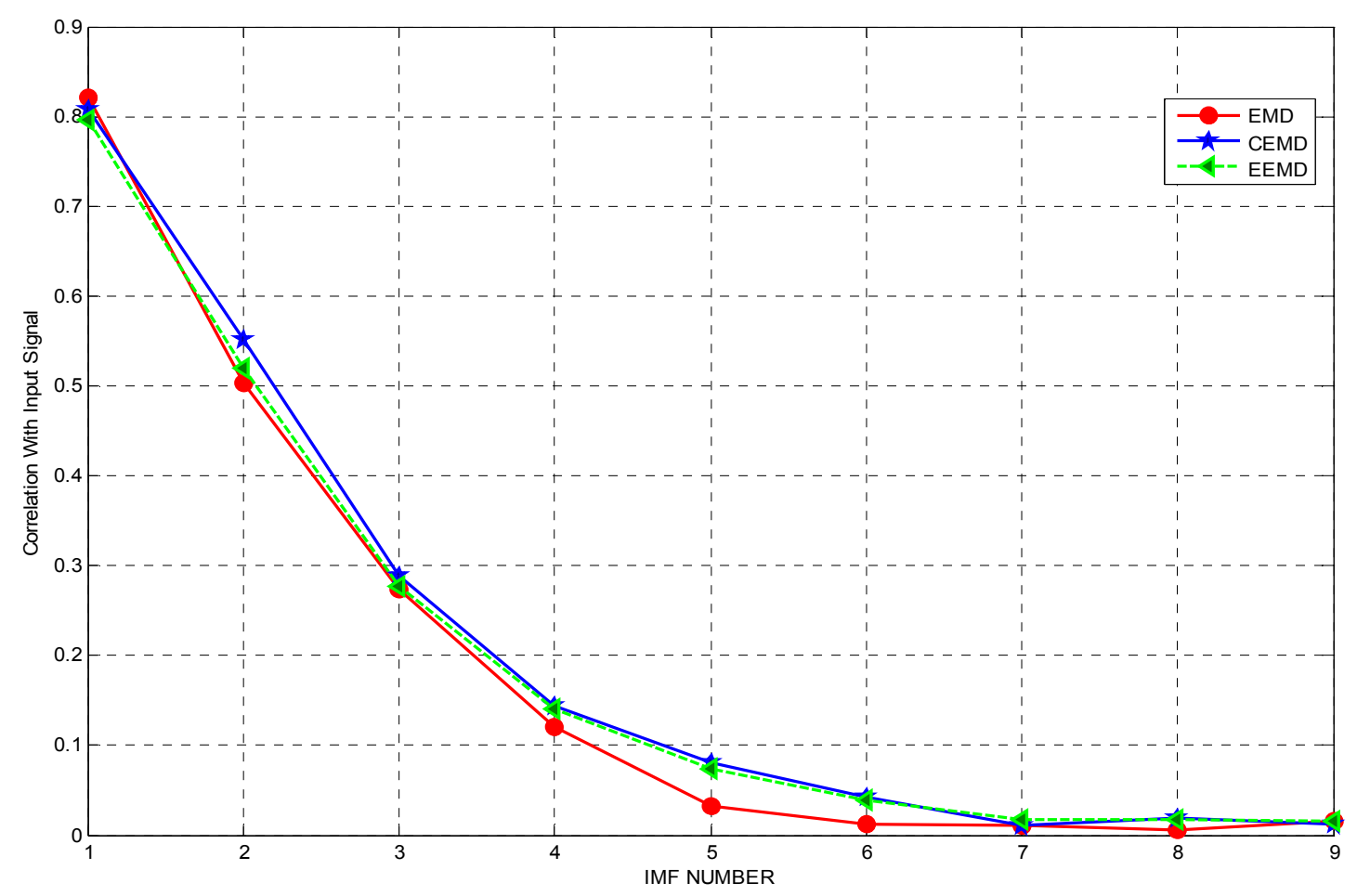

Figure 16: Correlation coefficients of IMFs with the input signal for the various methodologies (EMD, EEMD, and CEMD).

Correlation values for the first 5 IMFs are unstable although relatively high. The instability might be due to some amount of intermittency (very little because correlation is high) in the highest frequency modes. It stabilizes and almost flattens out as we approach the residual trend (last IMF). Also, it is important to note that all 
correlation values are above 0.5 which implies that corresponding IMFs from CEMD and EEMD contains almost the same physical meaning or information. Correlation values for the CEMD and EMD (figure 17B) are not very appreciating. The main contributing factor might be from the effect of intermittency or mode mixing on the EMD algorithm. As a result some information peculiar to IMFs (from CEMD and EEMD) are lost or shifted producing meaningless IMFs.
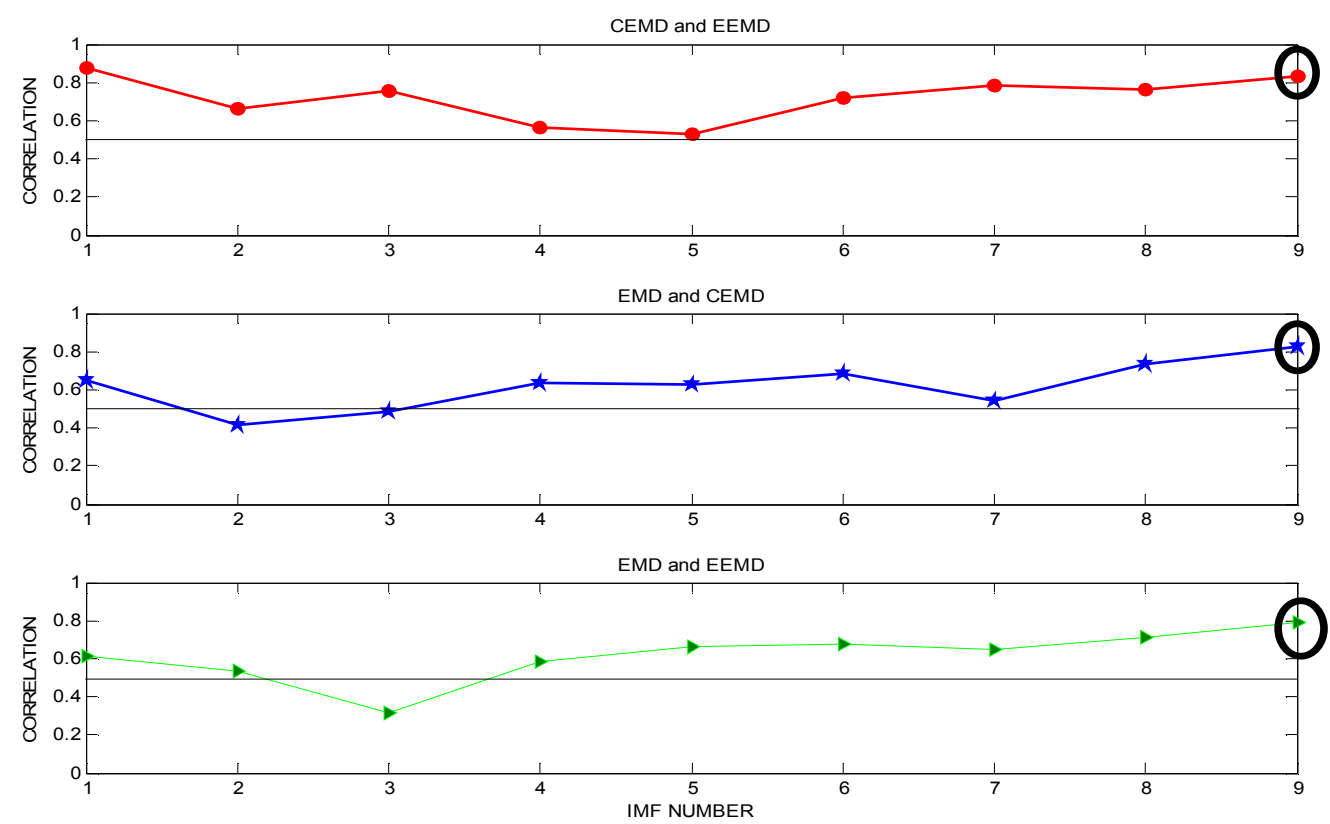

Figure 17: Correlation coefficients of corresponding IMFs for A) CEMD and EEMD. B). EMD and CEMD. C). EMD and EEMD.

It should be emphasized that although some of the IMFs lost some information from figure 17B, most of the correlation values were above 0.5 . 
Figure $17 \mathrm{C}$ also depicts almost the same relationship as figure $17 \mathrm{~B}$ with lower correlation values relative to figure $17 \mathrm{~A}$.

An interesting relationship is the high correlation values for the residual trend IMFs. All three figures depict this trend. This confirms that all the three methodologies CEMD, EEMD and EMD will perform creditably in trend analysis.

The results from the phase-locking concept reveal some additional information about the methodologies. For clarity, the phase locking analyses is focused on a section of our profile; about $100 \mathrm{~m}$ long. Phase locking values between the first IMF for the different methodologies is shown in figures $18 \& 19$. The plot reveals significant phase locking from 40-90m and from 0-10m. Surprisingly, both plots show similar relationship. All other subsequent IMFs are also very similar for all methodologies. This development could mean that the inability of a particular

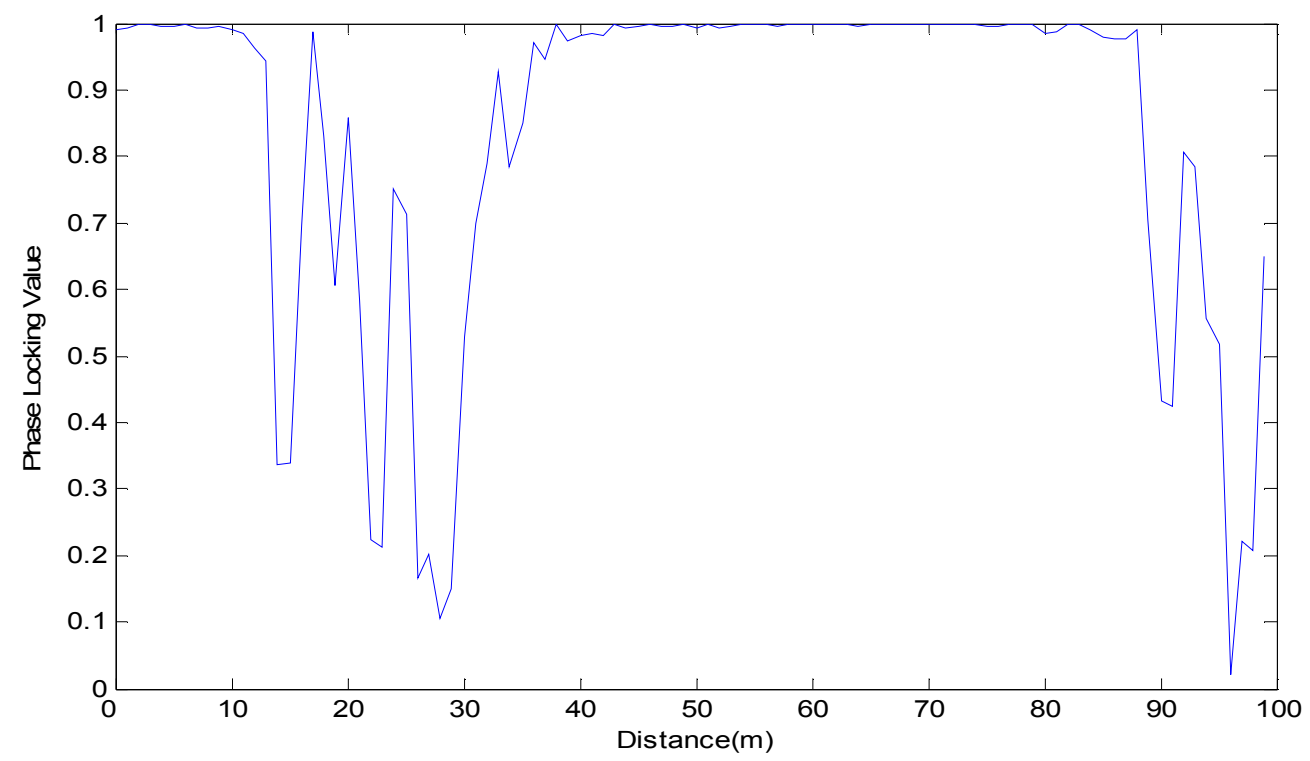

Figure 18: Phase locking values between the first IMF for EMD and EEMD. Reveals phase locking from $40-90 \mathrm{~m}$ and $0-10 \mathrm{~m}$. 


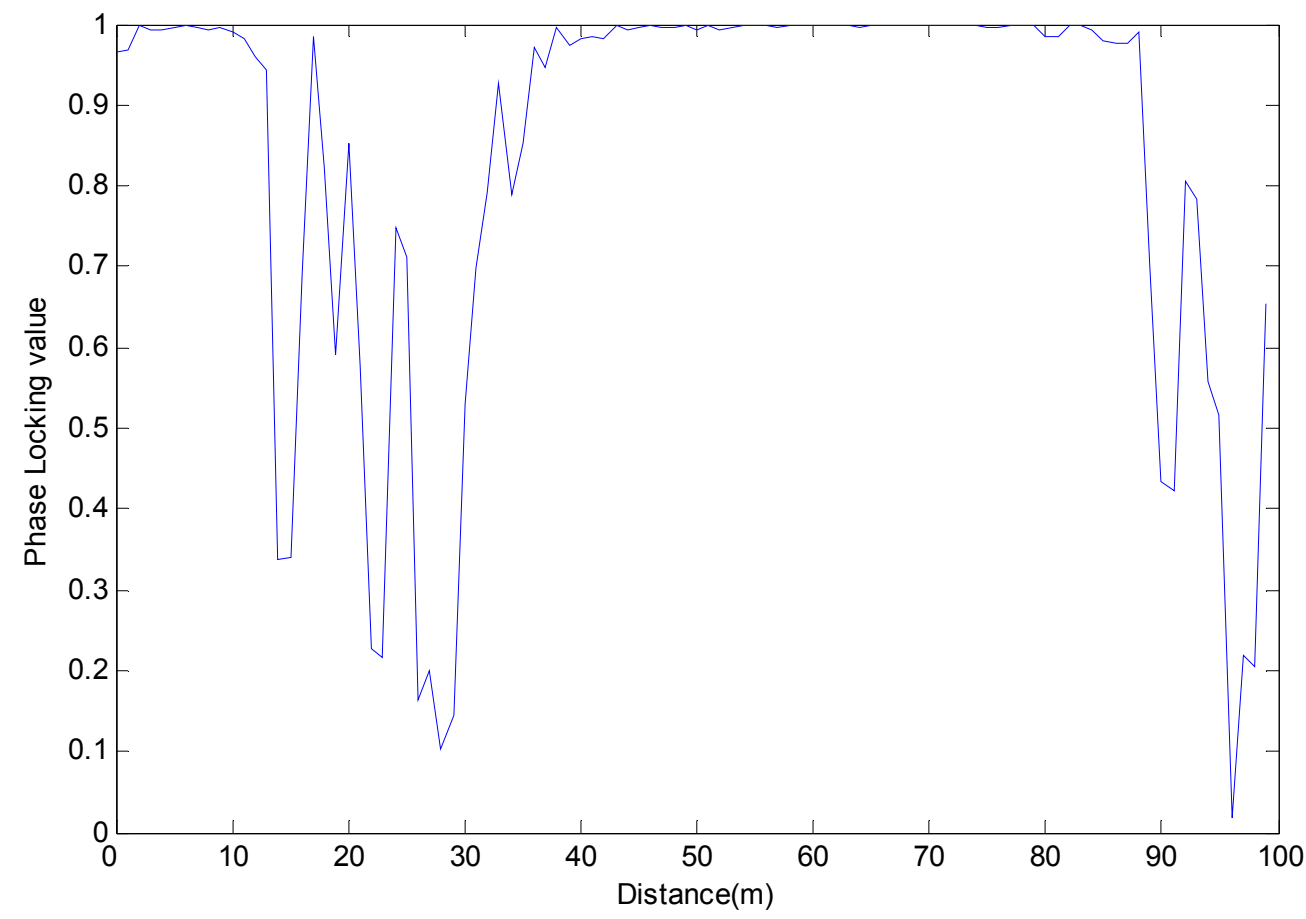

Figure 19: Phase locking values between the first IMF for CEMD and EEMD. Reveals phase locking from $40-90 \mathrm{~m}$ and $0-10 \mathrm{~m}$.

methodology to separate the individual IMF components perfectly due to mode-mixing can affect correlation values while phase relations may nonetheless be preserved. As the IMF number increases, the corresponding IMFs slowly become perfectly phase locked; thus the variations in phase decreases as we approach the residual trend. From figures $20 \& 21$, it is realized that complete phase locking begins as early as the fourth IMF. The remainder of the figures has been included in the appendix. 


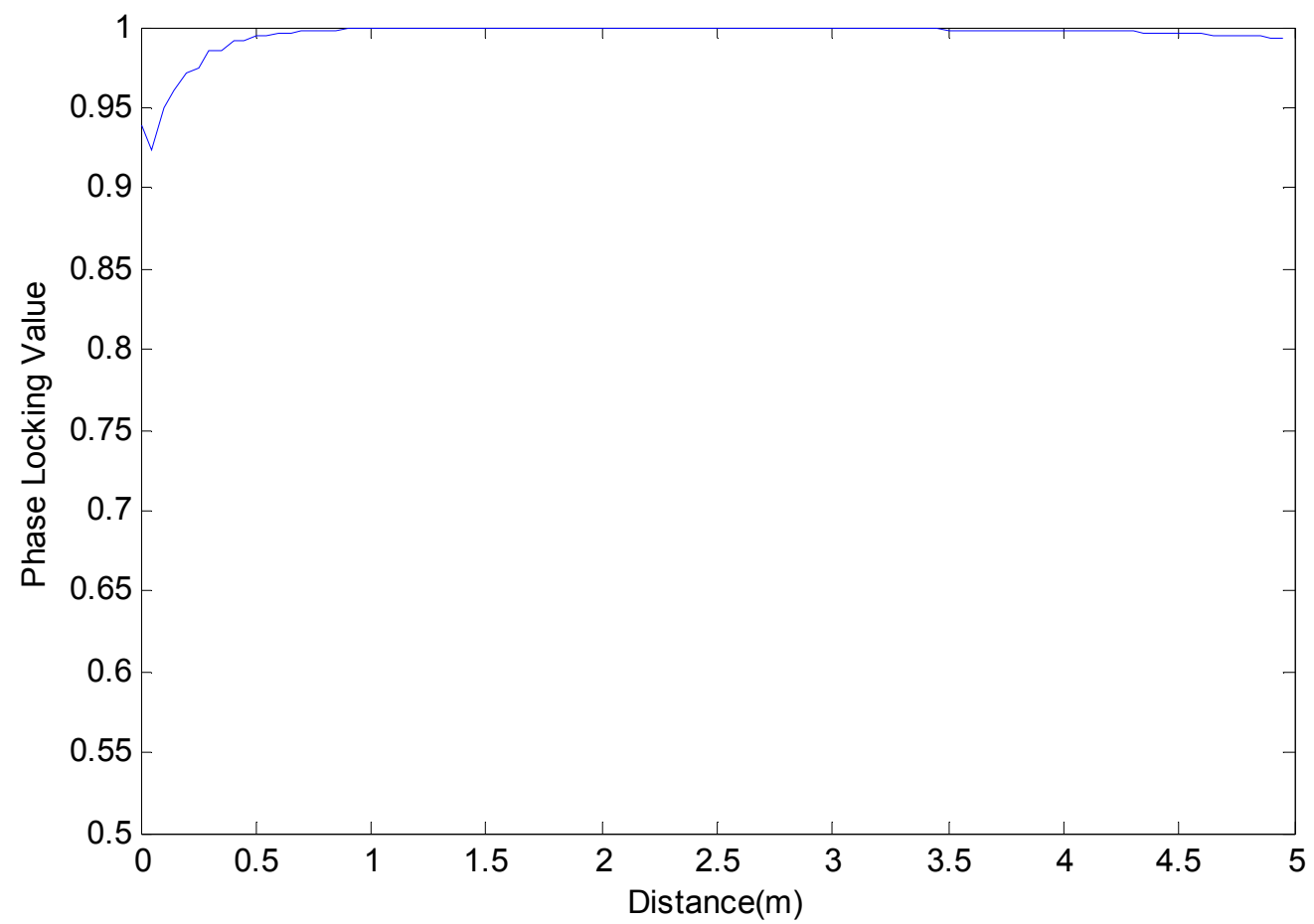

Figure 20: Phase locking values between the fourth IMF for EMD and EEMD. Component is almost completely phase-locked.

The energy-time-frequency space plot of the IMFs is displayed from figures 22-27. As explained earlier, the Hilbert Huang algorithms avoid shifting signal energy due to its localized information extractions. The figures confirm this claim; all the three algorithms located similar energy contents at particular sections on the profile. 


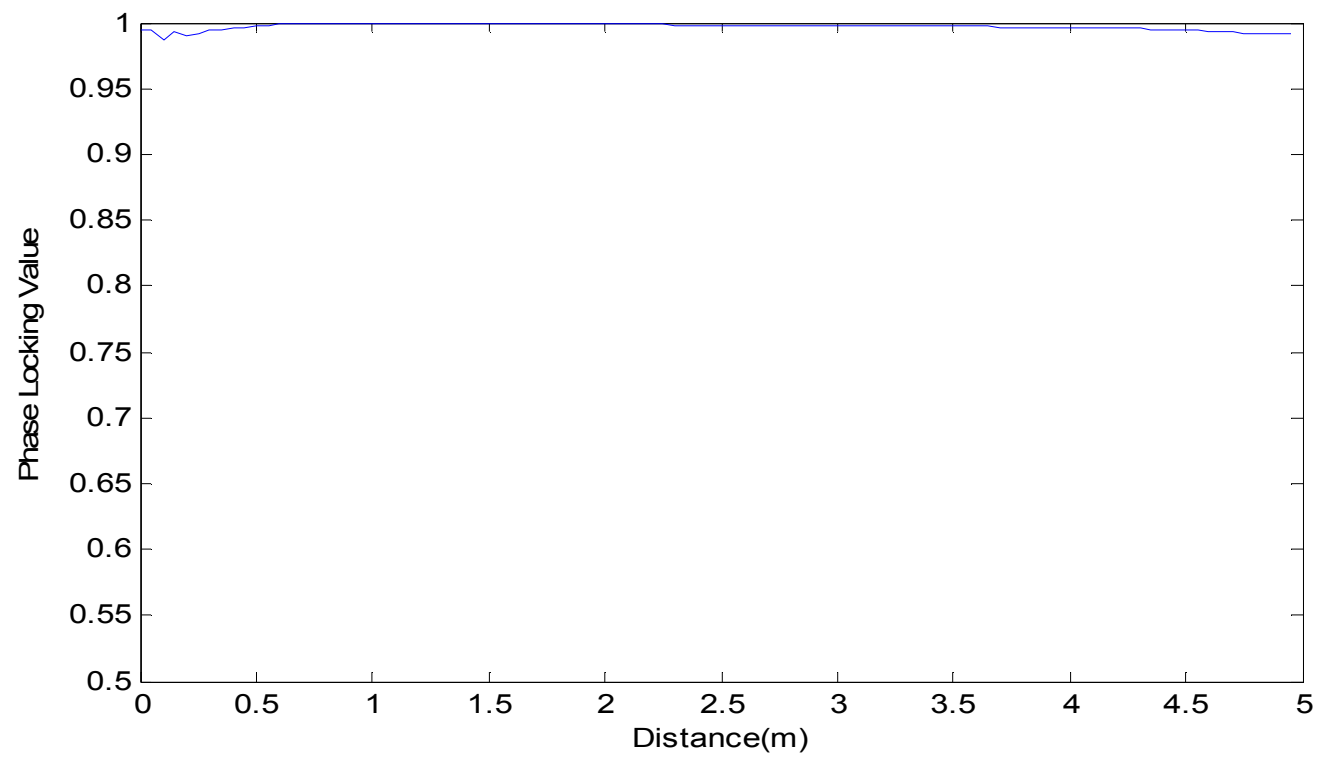

Figure 21: Phase locking values between the fourth IMF for CEMD and EEMD. Component is almost completely phase-locked.

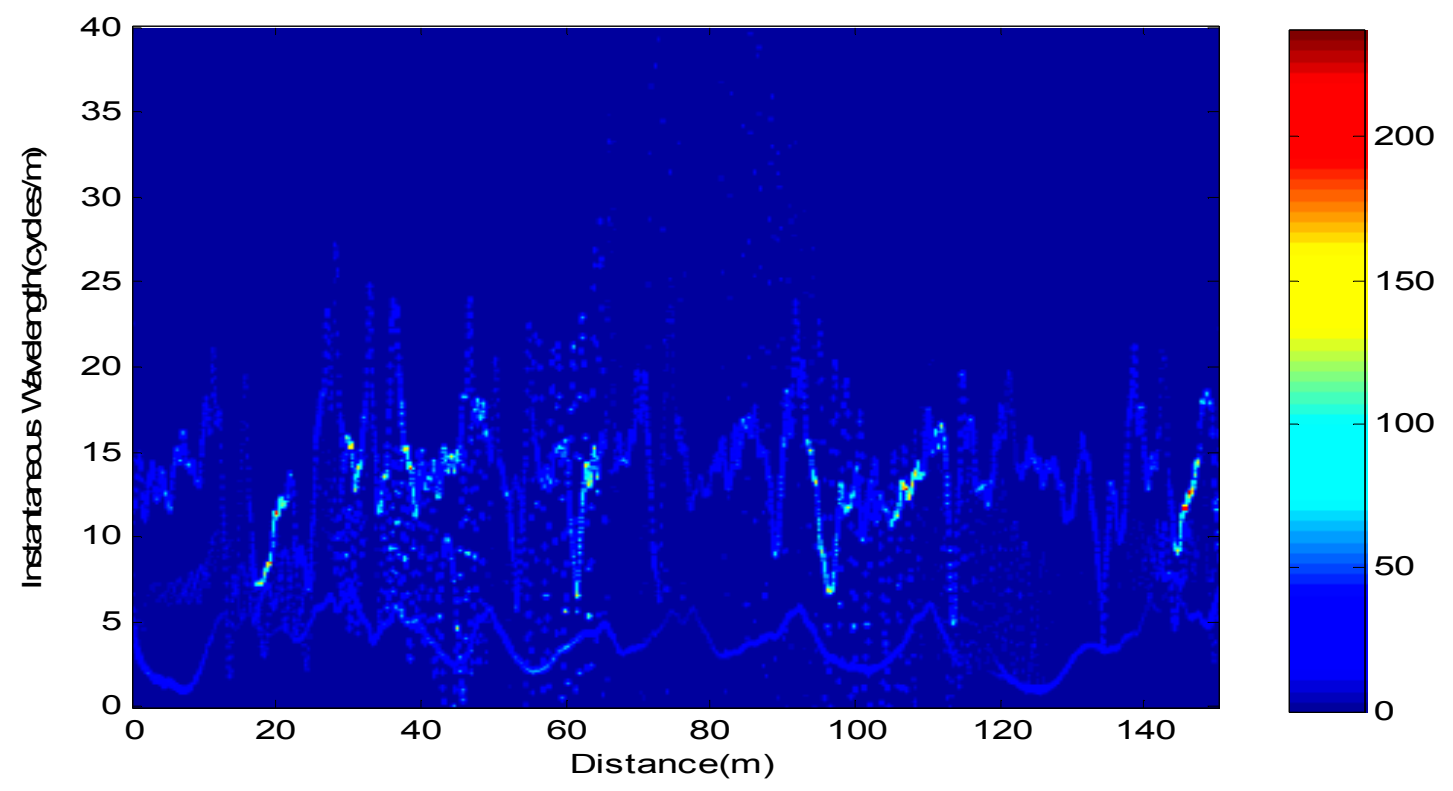

Figure 22: Energy-Distance-Frequency plot for IMFs 1-3 from EMD. 


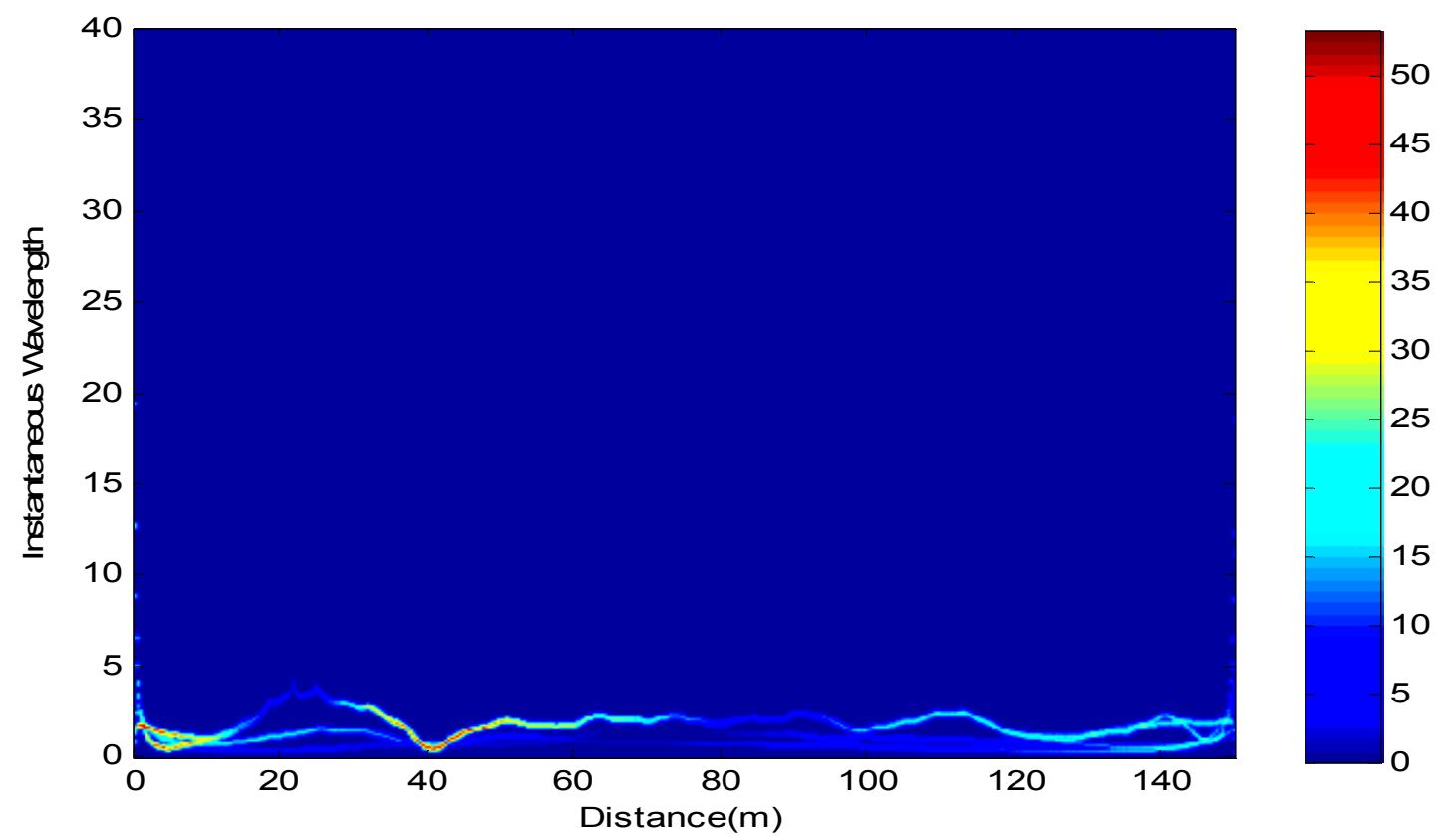

Figure 23: Energy-Distance-Frequency plot for IMFs 4-6 from EMD.

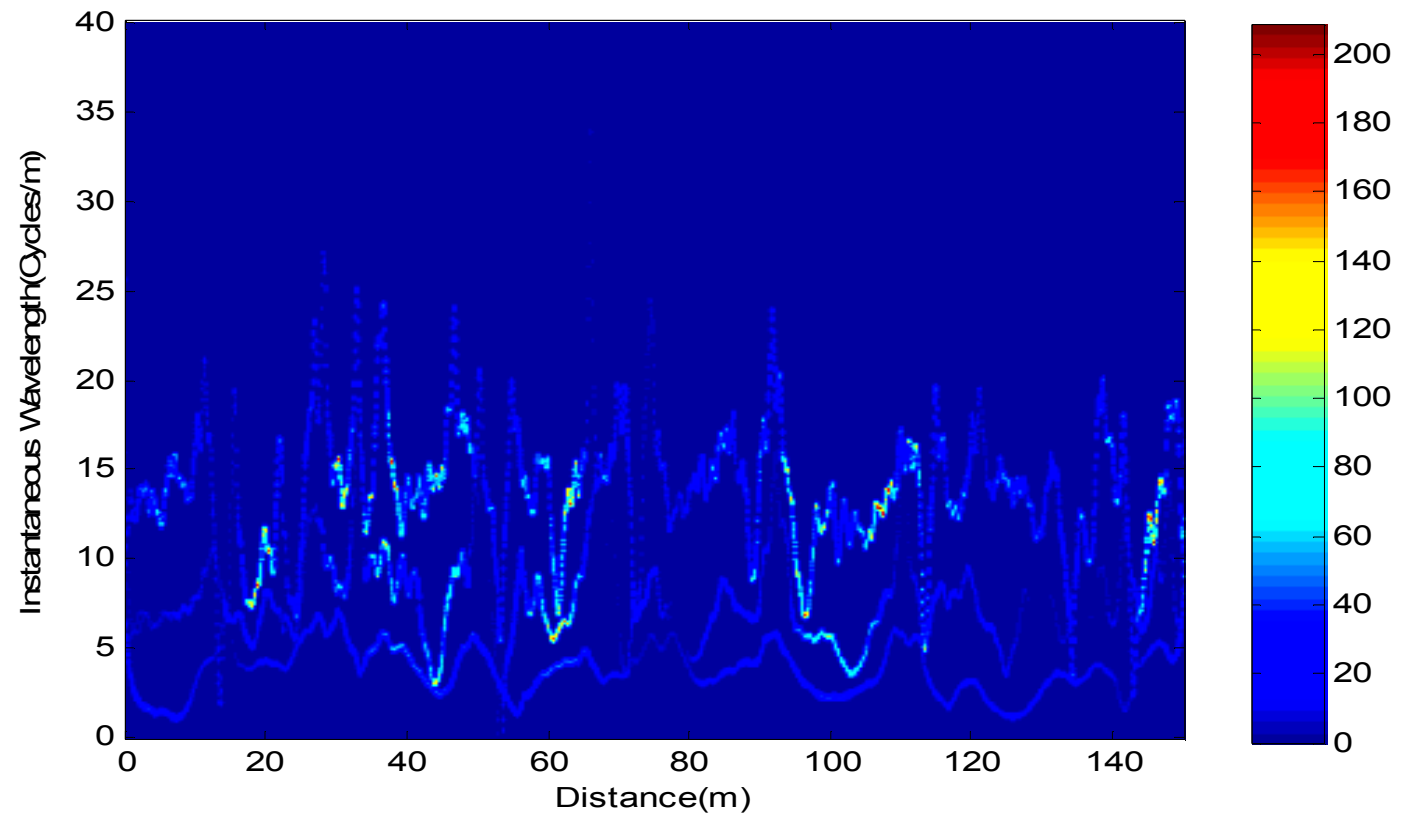

Figure 24: Energy-Distance-Frequency plot for IMFs 1-3 from EEMD. 


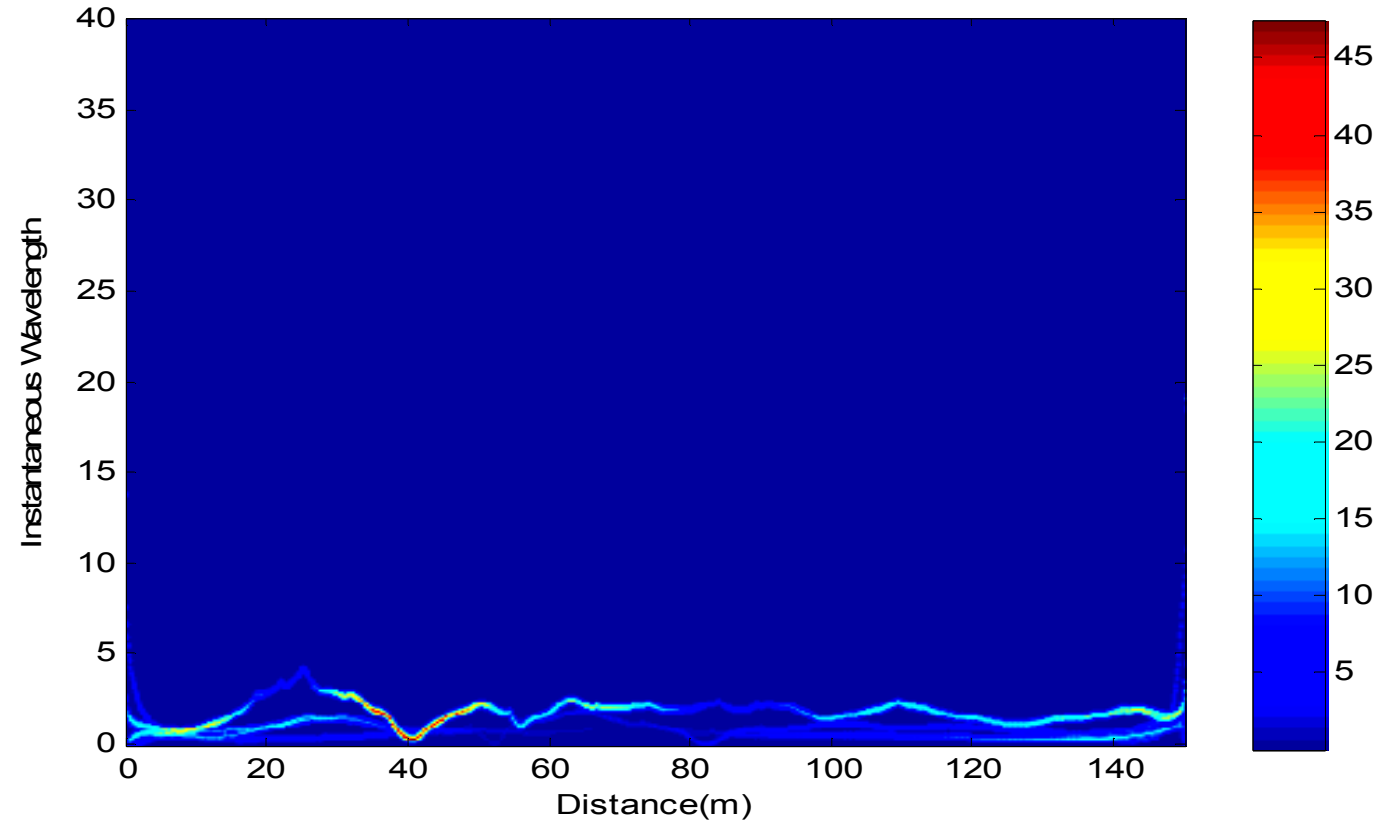

Figure 25: Energy-Distance-Frequency plot for IMFs 4-6 from EMD.

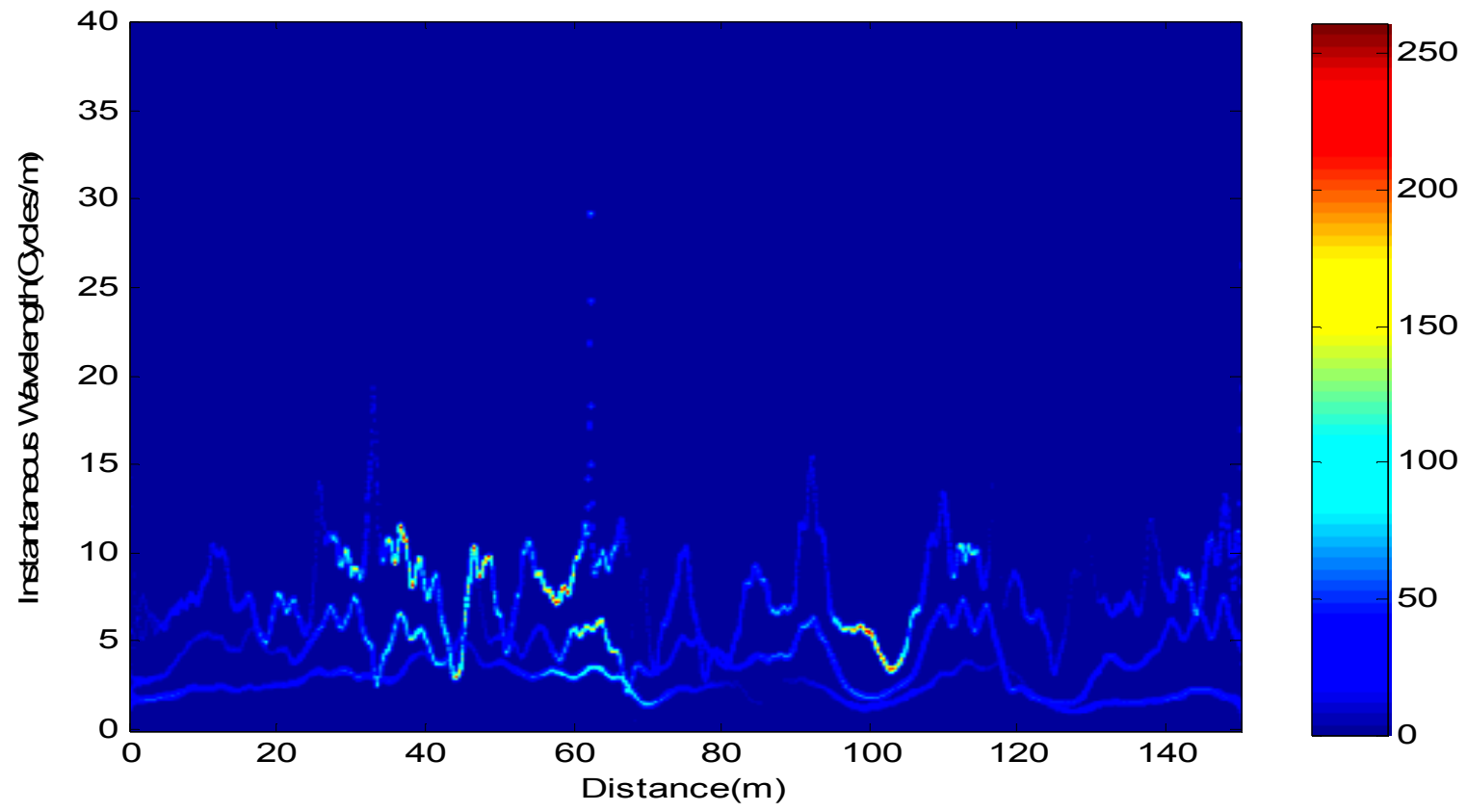

Figure 26: Energy-Distance-Frequency plot for IMFs 1-3 from CEMD. 


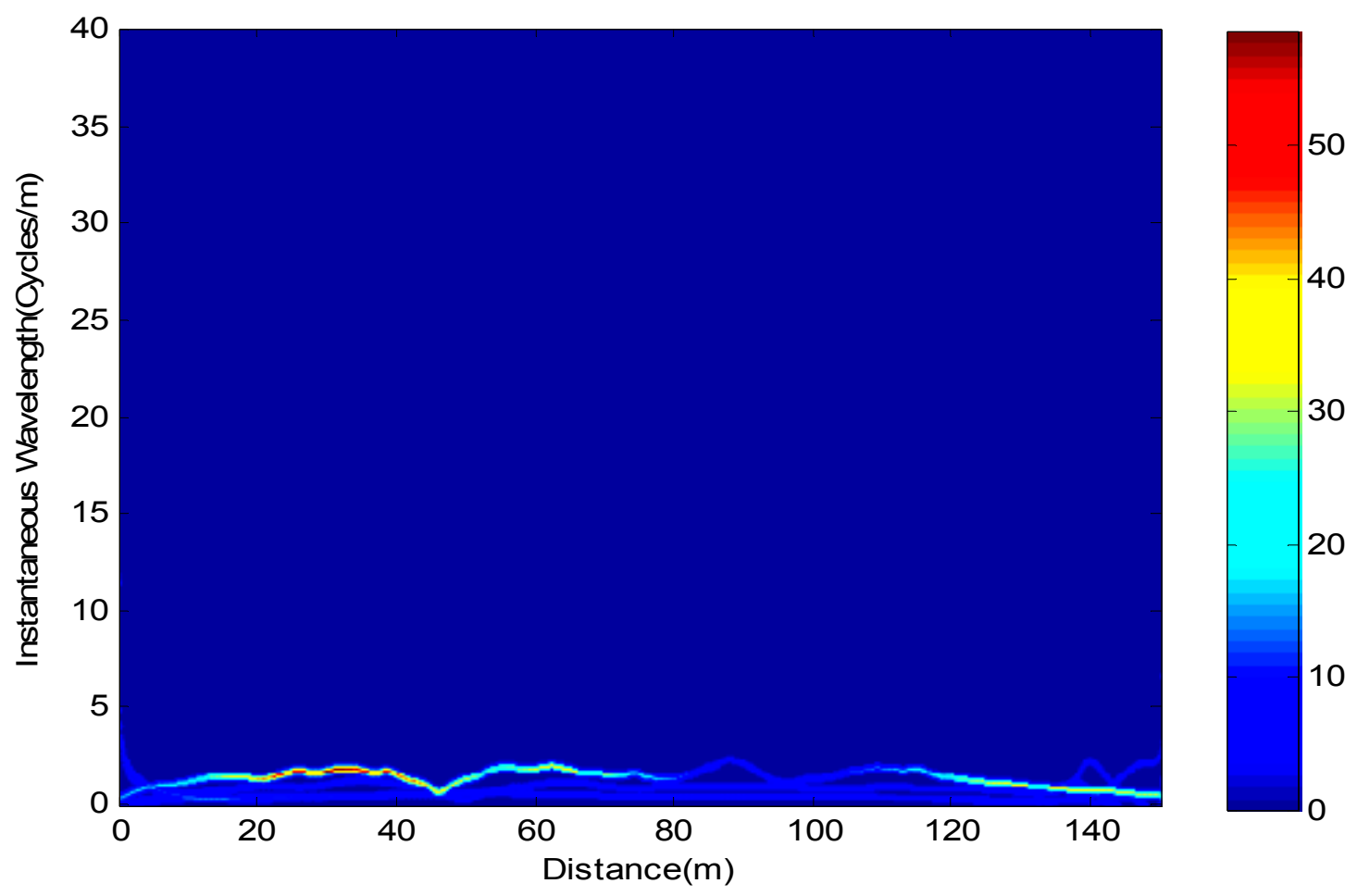

Figure 27: Energy-Distance-Frequency plot for IMFs 4-6 from CEMD.

The deductions above imply that although the EEMD's IMFs information is similar to the ones revealed by CEMD's IMFs in this case; in general, IMFs from the CEMD could be trusted against that from the traditional EMD and the EEMD due to its ability to significantly reduce mode mixing and suppress the effect of intermittent noise in our data. The next section describes how the CEMD was employed to extract some features from the profile.

\subsection{Energy-Gradient Based Reconstruction:}

Analysis of individual IMFs reveal some amount of information about the general trend and localized roughness information. However, some of these IMFs by 
themselves may not carry a well-defined physical meaning in profile analysis; carefully grouping modes with comparable features together can be useful for pattern recognition in the profile. In this section, a stage-by-stage energy-gradient based reconstruction is used to group and reconstruct the IMFs to extract information related to the roughness and deterioration trend of the profile. Figure 28 shows the mean energy levels of the IMFs.

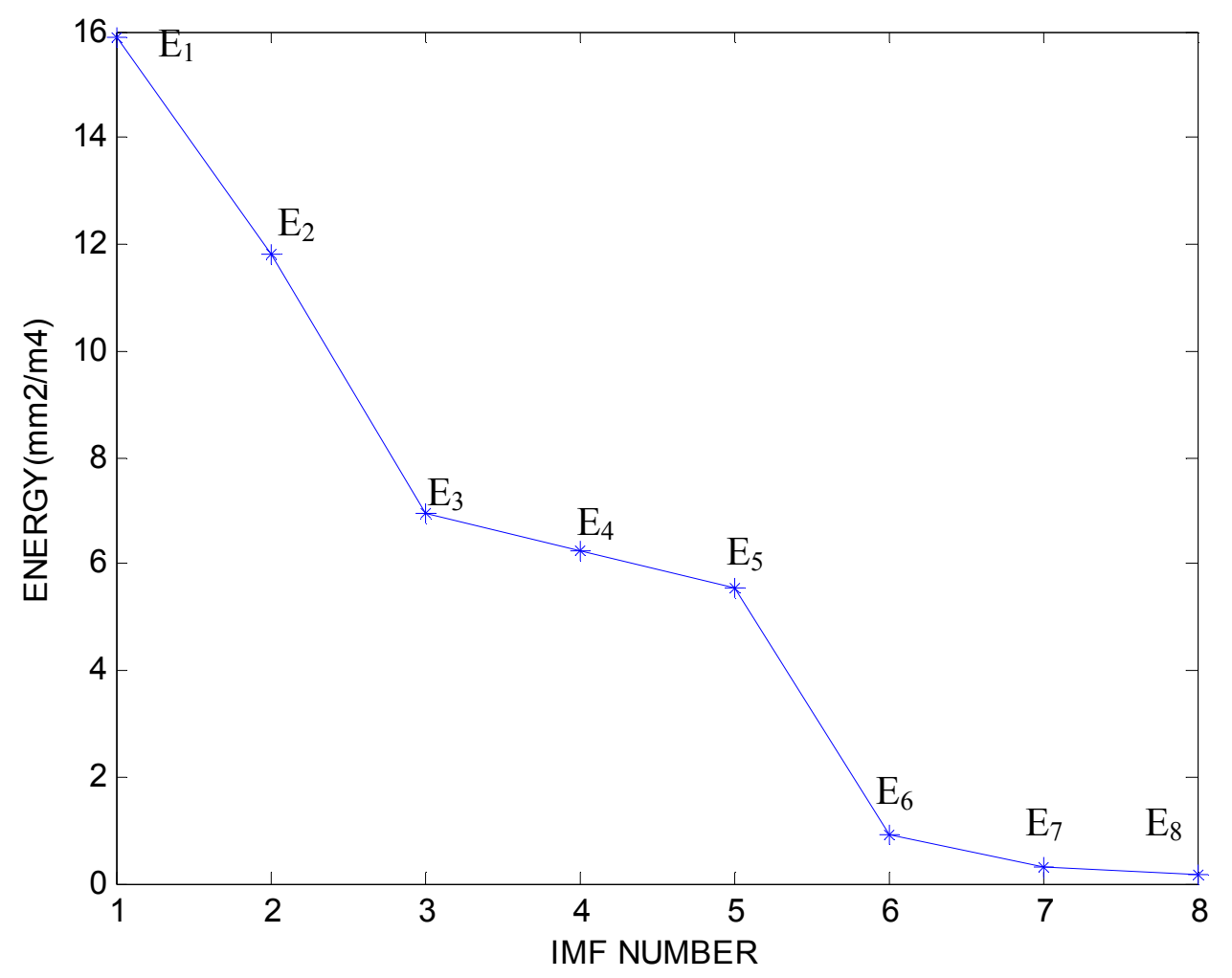

Figure 28: Plot of mean energy $\left(\times 10^{7}\right)$ against mode number

The energy-gradient is designated by the following steps:

Step 1: Determine the energy of the noise (the first IMF)

Step 2: Find the energy gradient from the noise to IMF2 as follows: 
$G E_{12}=\frac{1}{N+1} \Delta E$ where $\Delta E$ is the change in energy between IMFs $1 \& 2, \mathrm{~N}$ is the number of IMFs between them (zero in the current case), $G E_{12}$ is the energy gradient from IMFs 1-2.

Step 3: Find $G E_{23}$, if $G E_{23}>m * G E_{12}, m \geq 0.5$, then $G E_{23}$ and $G E_{12}$ is likely to characterizes different processes, else if $G E_{23}<m * G E_{12}$, then it is likely IMF2 and 3 characterize similar processes. The range of values for $\mathrm{m}$ is chosen to differentiate between gentle and steep gradients.

Step 4: find $G E_{34}$, if $G E_{34}<0.5 G E_{12}$, then $G E_{34}$ and $G E_{23}$ characterizes the same process, $G E_{34}$ falls into a different group. $G E_{12}$ is used as the reference gradient because the processes in IMF1\&2 are already known to be different (one is noise and the other is profile information) processes.

Step 5: repeat steps $3 \& 4$ for the remaining IMFs until they are all grouped

The basic assumption is that, sharp discontinuities (high gradient values) in energy of IMF components means that the modes characterize different processes in the profile.

The energy-gradient classification principle is now used to reconstruct IMFs with comparable energy and consequently for characterizing different features such as localized road roughness identification, extent and trend of deterioration. Since the energy $(k)$ of the IMFs to the original signal differs, reconstruction of the independent modes should be scaled by the respective contribution from each mode. The IMFs with comparative energy is reconstructed as follows:

1. Energy of the original signal is normalized to 1

2. IMF with comparative energy levels are reconstructed using the equation: $\sum_{\forall i} k C_{i}(t) \quad \mathrm{i}=1,2,3 \ldots$ Where $\mathrm{k}=\frac{E_{i}}{E_{s}}$, the Energy contribution from each $\mathrm{IMF}, E_{i}$ is the energy of the IMF and $E_{s}$ is the energy of the original signal. 


\subsection{Deterioration Pattern}

The pattern and extent of profile deterioration is an important index for pavement managers. In order to monitor this trend effectively, profile data for a period of years is required. The data employed in this analysis spans only one year, so the focus of the thesis in this area is to extract the IMFs that effectively capture the extent of deterioration for the particular year in question. The pattern of deterioration can easily be monitored with data for subsequent years available. The deterioration trend of the profile usually is a slow varying trend. Therefore it is expected to be captured by low frequency components and the residue. From the comparable gradient-energy reconstruction procedure, IMF 6-8 captures the deterioration trend of the profile. The estimated trend signal is designated as $X_{T}(t)=\sum_{\forall i} k C_{i}(t), i=6,7,8$

The wavelengths of these IMFs are between $20 \mathrm{~m}-50 \mathrm{~m}$. Clearly, there are some information derived from the reconstructed signal as shown in figure 29 that could not be found in a single IMF. The marginal spectrum in figure 30 gives us an indication which frequencies are dominant and contributes more in terms of energy to the deterioration trend. From the figure, the dominant frequency is center on approximately 0.01 cycle/meter. The corresponding power of this frequency although high might not cause much discomfort to road users due to the long wavelengths involved. However, sudden dips and humps at $35 \mathrm{~m}, 100 \mathrm{~m}$ and $145 \mathrm{~m}$ could cause a lot of vibration to a vehicle due to the amplitudes of these features. 


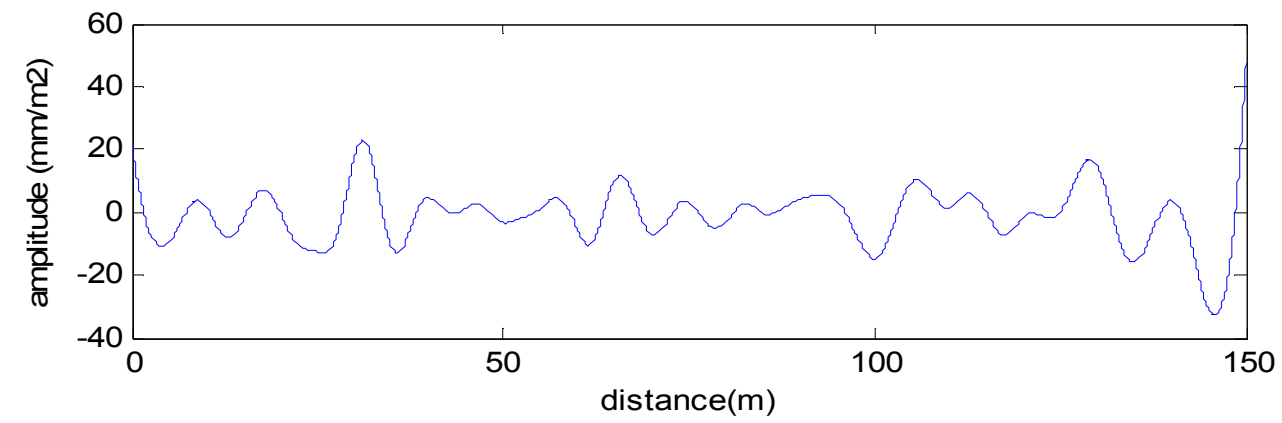

Figure 29: Reconstructed signal for the deterioration trend analysis

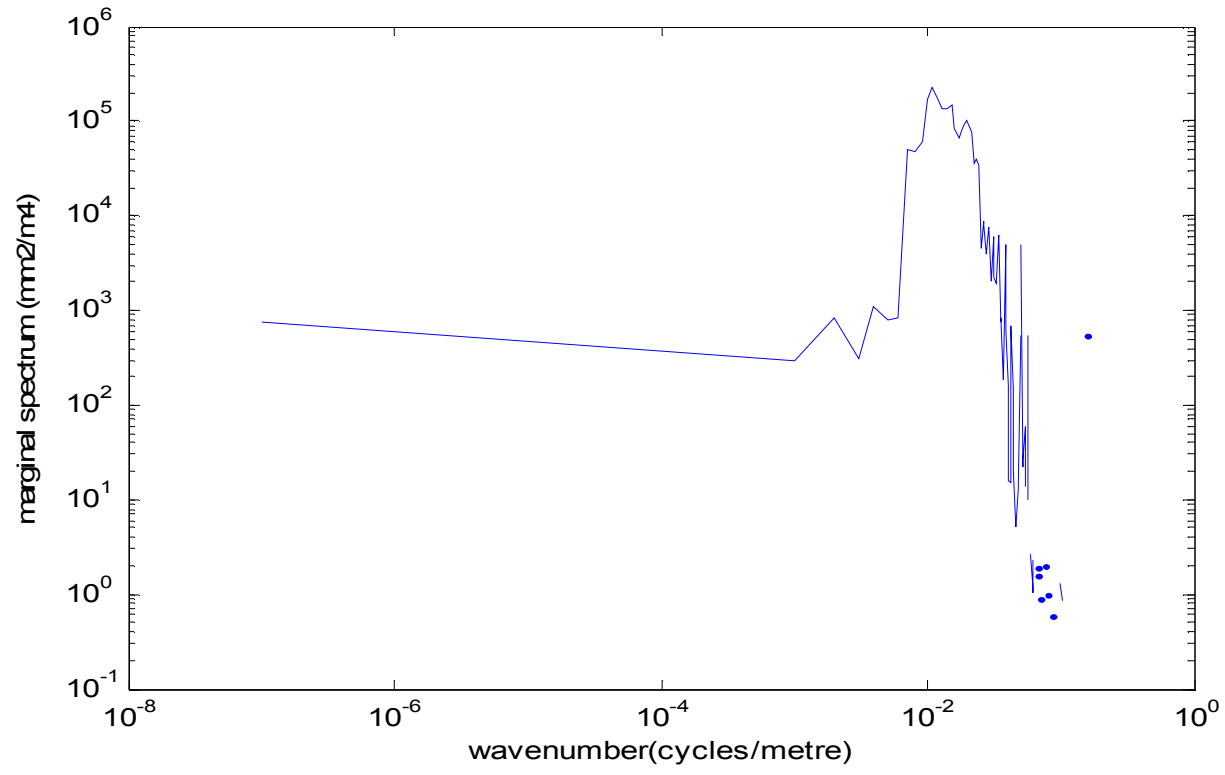

Figure 30: Marginal Spectrum of the reconstructed signal showing energy of dominant wavenumbers

\subsection{Localized Profile information}

The spatial rate of change of elevation remains the most important characteristic contributing to roughness. In order to accurately capture localized profile information, the algorithm employed should operate at the scale of every oscillation of 
the profile, without excessively mixing the scales. The CEMD provides profile analyst the opportunity to interpret results in terms of multiresolution analysis (energy-timefrequency), which aims at representing the process from most rough to the most subtle scale. In general, the algorithm is capable of identifying surface distresses even at wavelengths as low as $0.5 \mathrm{~m}$ and as long as $50 \mathrm{~m}$ (figure 13); mode mixing is also considerably reduced.

The energy-gradient classification method selected IMFs 3-5 as comparable scales that are contributing to the roughness of the profile. A scaled-sum of these IMFs is used to reconstruct the signal shown in figure 31.

$$
X_{R}(t)=\sum_{\forall i} k C_{i}(t), i=3,4,5
$$

The profile appears to be generally smooth; sudden peaks in the signal are likely to be joints due to the spacing between the peaks. Some of the joints might have been missed (from $0-25 \mathrm{~m}, 60-80 \mathrm{~m}$ and $120-140 \mathrm{~m}$ ) due to the profilometers sampling interval. From the marginal spectrum in figure 32, the wavelengths with stronger impact are centered on about 1 cycle/meter. The range which most affects how a pavement feels to riders in a passenger car is when there is more than one cycle in a distance between 1.4 and 220 feet $(0.43$ and $67 \mathrm{~m})$ (Karamihas 2005). The marginal spectrum reveals that, for this profile there is more than 1 cycle in every $10 \mathrm{~m}$ on the average. This of course will affect the comfort of road users. 


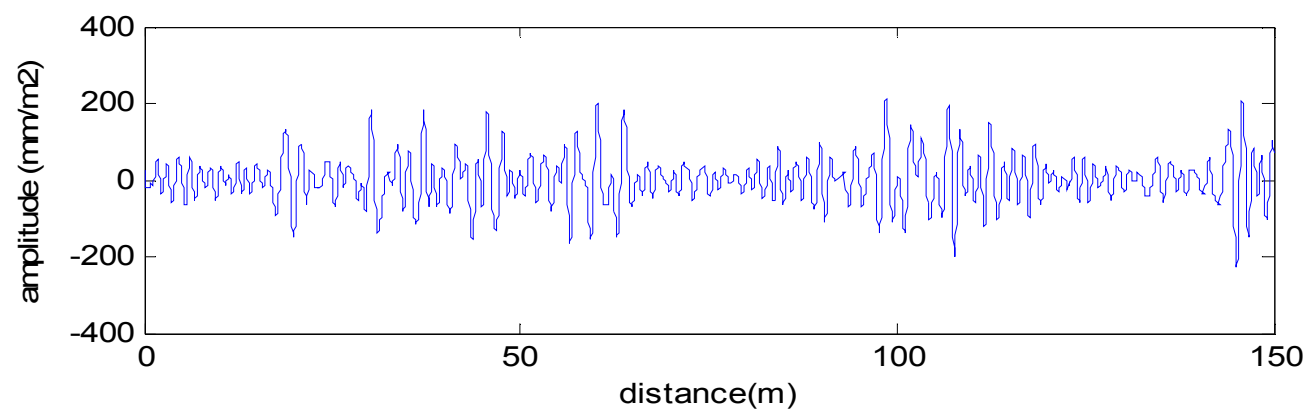

Figure 31: Reconstructed signal for localized profile information

The level of detail provided for the profile analyst when mode mixing is removed is incredible. A typical example is displayed in figure 33 showing an IMF and its corresponding energy-time-frequency space distribution. This representation provides useful information especially for network pavement profile analysis; it is very important to know where the dominant frequencies are located as well as the energy of the dominant frequencies along the pavement section. The domain of energy concentration for the IMF is indicated on the color bar. . 


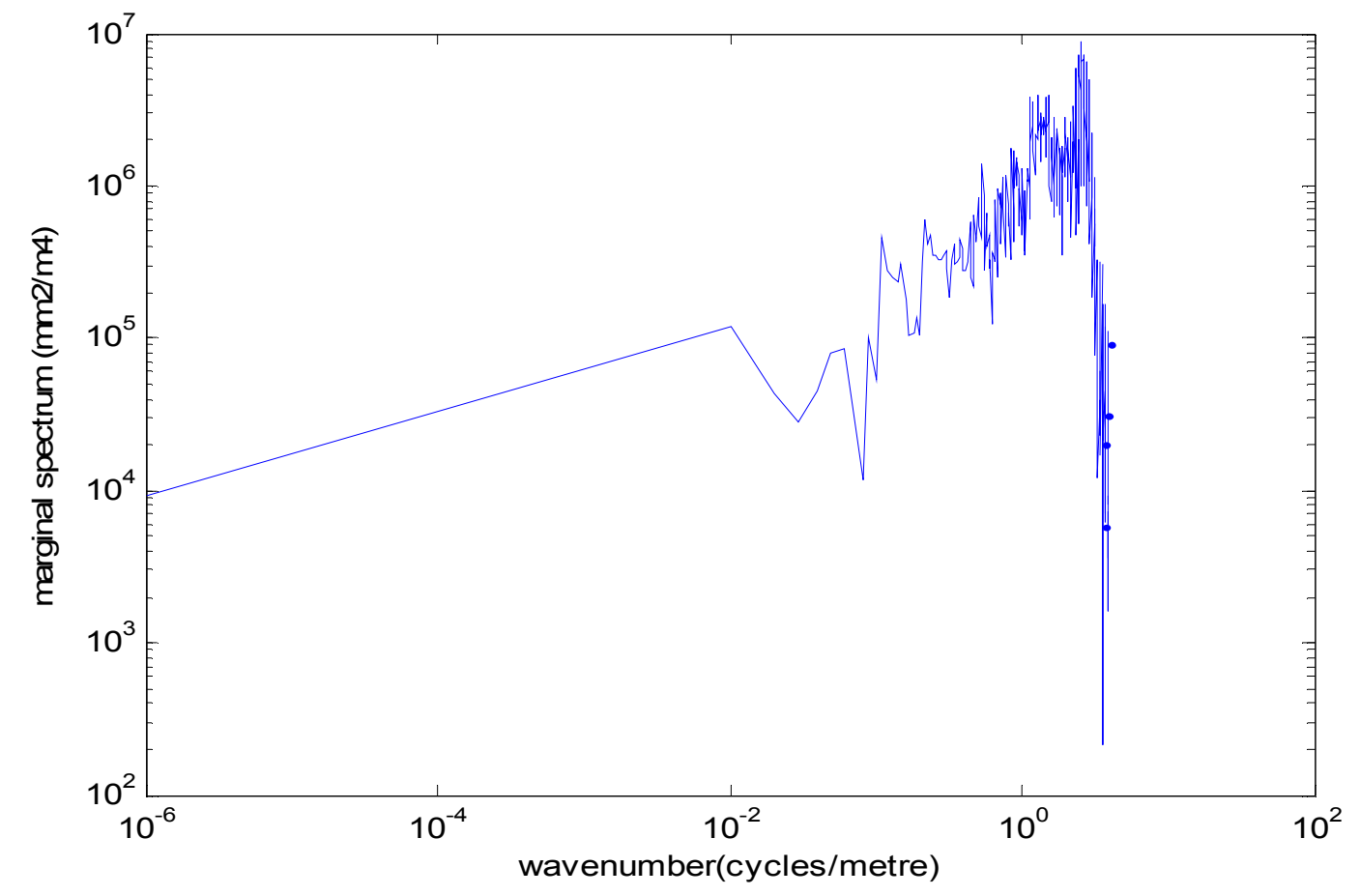

Figure 32: Marginal Spectrum of the reconstructed signal showing energy of dominant wavenumbers

Mode mixing shifts signal energy and distort the ability to accurately describe the energy-time-frequency distribution of the profile. Most methods for profile analysis fail at this point. 

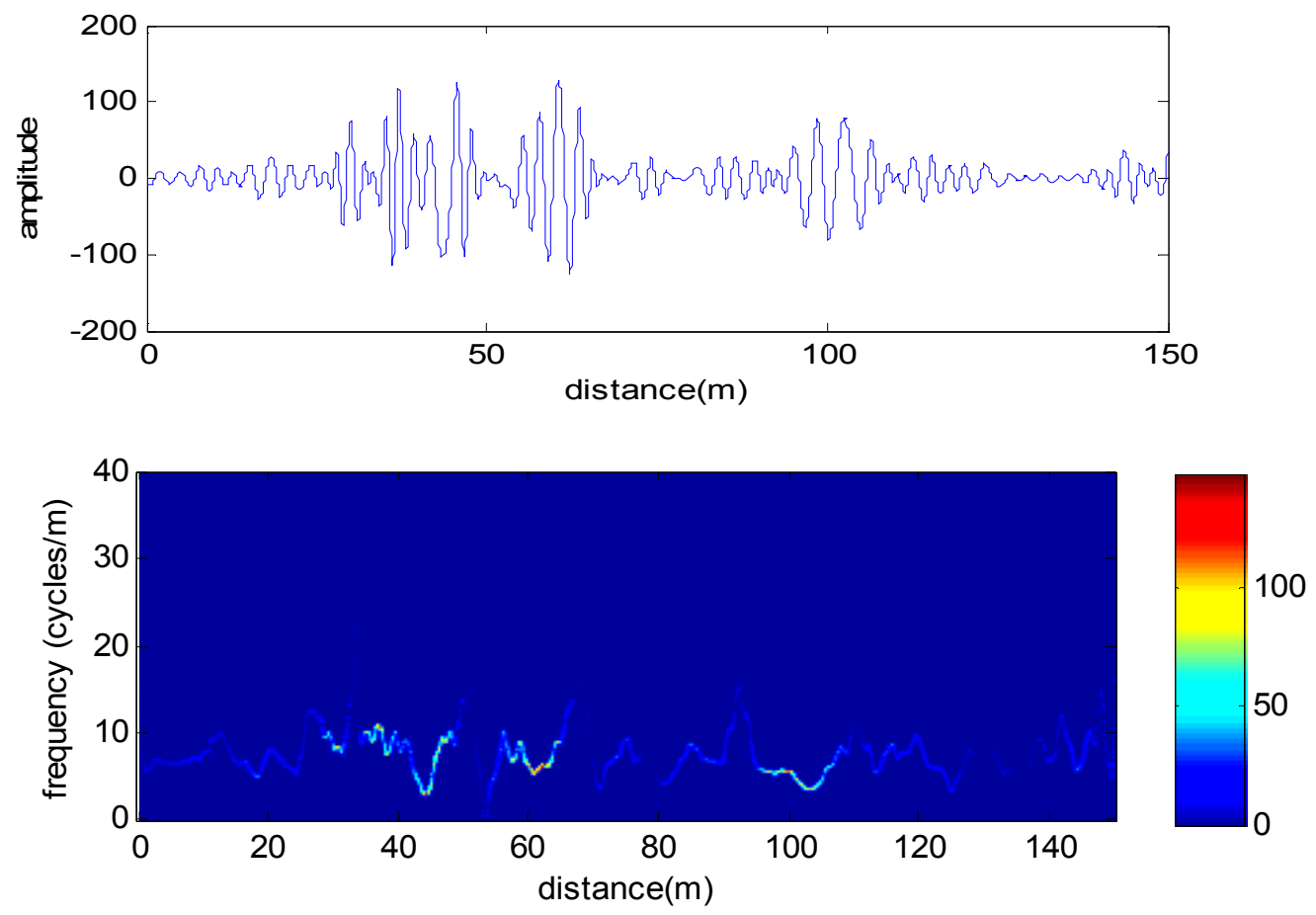

Figure 33: Energy-distance-frequency (below) diagram for a selected IMF (above) 


\section{Chapter 5}

\section{CONCLUSIONS AND RECOMMENDATIONS}

\subsection{General}

This study has explored the capabilities of using the different Hilbert Huang Algorithms for pavement profile analysis. It has been established that useful additional information could be obtained from pavement profiles, which are normal roughness records measured by typical profilometers. Such additional information is currently not obtainable from existing methods such as the IRI, Wavelet or the Power Spectral Density. A review of the existing dominating processes and methods for pavement profile analysis was used as a background for introducing the prowess of the HHT.

\subsection{Conclusion}

Based on the analysis of results presented in this thesis, the following conclusions and recommendations can be drawn:

The HHT is well-suited and an innovative process for analyzing nonstationary pavement profiles because it provides localized information of the road profile. The algorithm has been used for identification of patterns in profiles and to extract information such as phase, frequency (temporal information) and energy content along the longitudinal section of the profile. Unfortunately, the HHT is fraught with challenges especially at the first component level, which is the signal decomposition process. From this study, it is concluded that the mode mixing problem 
which is normally associated with the EMD process causes the algorithm to extract spurious IMFs falsely characterizing the underlying processes. Obtaining physically meaningful IMFs which truly characterize information within our data requires that the effect of intermittent noise is suppressed and the general residual trend is accurately estimated.

The CEMD outperforms the EEMD and EMD in terms of noise suppression and the effect of mode mixing as it uses information obtained from the correlated signals (real and imaginary) hence, its IMFs can be trusted for profile analysis. An extended development from this observation was that, although EMD was unable to separate the individual components perfectly due to mode mixing, their phase relations were perfectly preserved. The phase locking analysis of the corresponding IMFs confirms this development. The inability of the EMD algorithm to accurately separate IMFs due to mode-mixing did not affect the phase relations of the components. This suggests that all the algorithms will produce similar results if it is targeted at temporal analysis of pavement profiles.

Finally, our work has shown that all methodologies can be used to accurately estimate the residual trend. This characteristic was clearly displayed in the correlation and phase-locking analysis. Implicitly, this suggests that the algorithm can be employed to monitor the roughness deterioration trends of pavement sections, and changes in roughness contents brought about by pavement rehabilitation. An energygradient classification process was used to obtain physically meaningful information from the IMFs which truly characterize information within the dataset. The presented work has shown that the CEMD can be used to accurately estimate localized profile information, the extent and pattern of the deterioration trend. 
The methodology can also be applied to the structure health monitoring of other civil infrastructure systems by employing its damage identification and diagnosis capacity. It is also a useful tool for extracting useful information for network pavement management and pavement maintenance operations.

\subsection{Recommendations and Future Research}

- The implications and influence of HHT analysis in studies of pavement surface profiles at the different scales (mega, macro, and micro) need be investigated further to increase the level of accuracy and precision in estimating roughness, macrotexture, and other pavement parameters

- Both longitudinal and cross-section information should be employed in pavement profile analysis. Using one dimensional information implies that we need to make assumptions concerning how the profile will oscillate (single or bivariate) in the amplitude-distance space. This problem is absolutely resolved when the profile is decomposed as two-dimensional. A multidimensional empirical mode decomposition approach is suggested in this direction. Also for excellent visual interpretation of the profile changes, a three-dimensional view of the profile is required.

- Investigate relationships between profiling data, environmental data such as tire/pavement interaction noise and pavement surface data such as skid and macro texture data.

- Further research should be targeted at employing the capabilities of phase locking to identify defects, bumps and dips. 


\section{REFERENCES}

[1] Altaf, M.U., Gautama, T., Tanaka, T., and Mandic, D.P. (2006). "Rotation Invariant Empirical Mode Decomposition." IEEE Int. Conf. Acoustics, Speech, Signal Processing, Toulouse, France, Vol. 3, pp.1009-1012

[2] Ayenu-Prah, A.Y., Attoh-Okine, N.O. (2008). "Comparative study of Hilbert-Huang Transform, Fourier Transfom and Wavelet Transform in Pavement Profile Analysis." Vehicle System Dynamics, Vol. 47(1), pp.437-456

[3] Bruscella, B., Rouillard, V., and Michael, S. (1999). "Analysis of Road Surface Profiles." Journal of Transportation Engineering, Vol. 125(1), pp.5558

[4] Budras, J. (2001). "A Synopsis on the Current Equipment Used for Measuring Pavement Smoothness." FHWA Pavement Technology.

[5] Chang, J.R., Su, Y.S. Huang, T.C., Kang, S.C and Hsieh, S.H. (2009). "Measurement of IRI Using an Autonomous Robot Measurement of IRI Using an Autonomous Robot." $26^{\text {th }}$ International Symposium on Automation and Robotics in Construction, 
[6] Depont, J.J., and Scott, A. (1999). "Beyond Road Roughness-Interpreting Road Profile Data." Road and Transportation Research, Vol.8 (1) pp.2-28

[7] Dore, G., Flamand, M., and Pascale, P. (2002). "Analysis of the Wavelength Content of the Longitudinal Profiles for C-LTPP Test Sections.” Can. J. Civ. Eng. 29, pp. 50-57.

[8] Farnbach, J.S. (1975). "The Complex Envelope In Seismic Signal Analysis.” Bulletin of the Society of America, Vol. 65 (4), pp.951-962.

[9] Flandrin, P., Goncalves, P. and Rilling, G. (2004). "Detrending and Denoising with Empirical Mode Decompositions." IEEE Signal Process. Pg.1581-1584.

[10] Gabor D. (1946). “Theory of Communication.” J. IEE (London), Vol. 93, Part III, No. 26, Page 429-457

[11] Gagarin, N., Huang, N., Oskard, M.S. and Sixben, D.G. and Mekemson, J.R. (2004). "The Application of the Hilbert Huang Transform to Analysis of Inertial Profile of Pavements." Int. Journal of Vehicle Design. Vol. 36, pp.289.

[12] Huang, N.E., Wu, M.L., Long, S.R., Shen, S.P., Qu, W.D., Gloersen, P., and Fan, K.L. (2003). "A Confidence Limit for the Empirical Mode Decomposition and the Hilbert Spectral Analysis." Proc. Royal Society, London, Series A, Vol. 459, pp. 2317-2345. 
[13] Huang N.E., Shen Z., Long S.R., M.C. Wu, H.H. Shih, Q. Zheng, N-C. Yen, C.C. Tung and Liu, H. (1998). "The Empirical Mode Decomposition and the Hilbert Spectrum for Nonlinear and Non-Stationary Time Series Analysis." Proc. Royal Society London, Series A, Vol. 454 (1971), pp. 903-995.

[14] Karamihas, (2005). S.M., "Critical Profiler Accuracy Requirements." Draft Final Report, Transportation Pooled Fund Program, Project No. TPF 5(063), University of Michigan Transportation Research Institute, Ann Arbor, MI,

[15] Klionski, D.M., Oreshko, N.I., Geppener, V.V., and Vasiljev, A.V. (2008), "Applications of Empirical Mode Decomposition for Processing Nonstationary Signals." Pattern Recognition and Image Analysis, Vol.18 (3), pp. 390-399.

[16] Loutridis, S.J. (2004). "Damage Detection in Gear Systems Using Empirical Mode Decomposition.” Engineering Structures, Vol. 26(12), pp. $1833-1841$.

[17] Marcondes, J. (1991) “Spectral Analysis of Highway Pavement Roughness.” ASCE Journal of Transportation Engineering, Vol. 117(5), pp. $540-549$

[18] OCDE, (1984). “Caractéristiques des Revêtements Routiers: Leur Intéraction et Leur Optimization, Recherche en Matière de Routes et de 
Transports Routiers." Organisation de Coopération et de Développement Économique (OCDE), Paris, France,

[19] Perera, R.W. and Kohn, S.D. (2002). "Issues in Pavement Smoothness: A Summary Report." NCHRP Web Document 42 (Project 20-51[1]):

Contractor's Final Report, March

[20] Rilling, G., Flandrin, P., Goncalves P., and Lilly J. M. (2007). "Bivariate Empirical Mode Decomposition." IEEE Signal Processing Letters, Vol. 14 (12), pp. 936-939

[21] Rouillard, V. Sek, M.A. and Perry, T. (1996), “Analysis and simulation of road profiles." ASCE J. Transp. Eng. 122(3), pp. 241-245.

[22] Sayers, M.W. and Karamihas, S. M. (2003). "The Little Book of Profiling." University of Michigan Transportation Research Institute.

[23] Spangler, E.B. and Kelly, (1966). "W.J. GMR Road Profilometer-A Method for Measuring Road Profile." Highway Research Record Vol. 121, pp 27-54. 
[24] Sweeney-Reed C.M., Nasuto S. J. (2007). "A novel approach to the detection of synchronisation in EEG based on empirical mode decomposition." J Comput Neurosci, Vol 23, pg. 79-111,

[25] Tanka, T. Mandic, D.P. (2007). "Complex Empirical Mode Decomposition." IEEE Int. Conf. Acoustics, Speech, Signal Processing, Vol.14 (2), pp.101-104

[26] WU, Z., Huang, E. (2005), "Ensemble Empirical Mode Decomposition A Noise-Assisted Data Analysis Method." Calverto: Center for Ocean-LandAtmosphere Studies, pp. 1-51.

[27] Yaguo, L, Zhengjia, H, Yanyang, Z. (2009). "Application of the EEMD Method to Rotor Fault Diagnosis of Rotating Machinery." Mechanical Systems and Signal Processing, Vol.23 (4), pp.1327-1338,

[28] Zhou, F.., Xing, M.., Bai X., Sun, G., and Bao, Z. (2009). "Narrow-Band Interference Suppression for SAR Based on Complex Empirical Mode Decomposition." IEEE Geoscience and Remote Sensing Letters, Vol.6 (3), pp. 423-427.

[29] Zhou, X. Zhao, H. and Jiang, T. (2009). "Adaptive analysis of Optical Fringe Patterns Using Ensemble Empirical Mode Decomposition Algorithm." Optics Letters, Vol.34 (13). 


\section{APPENDIX I}

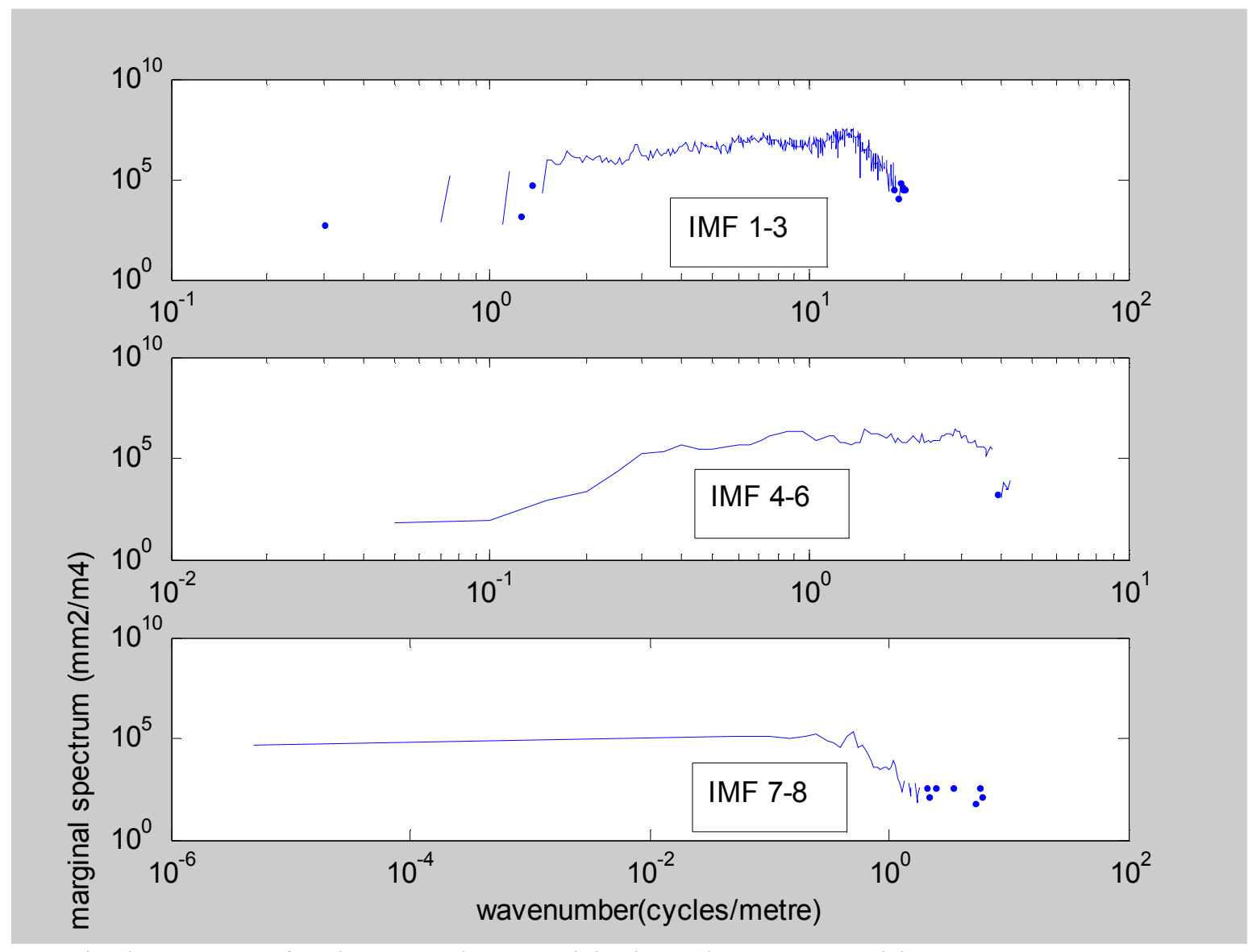

Marginal Spectrum for the Complex Empirical Mode Decomposition 


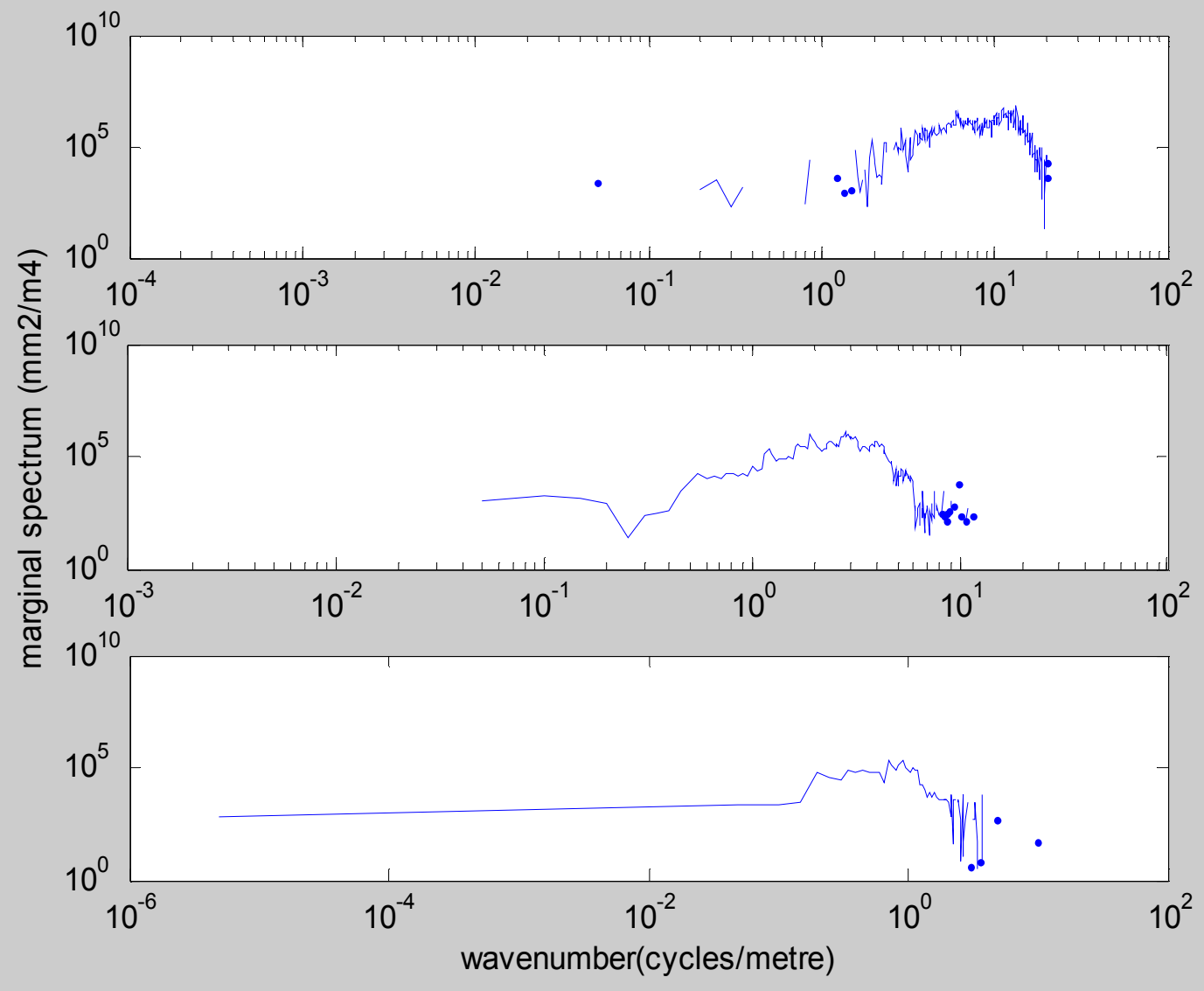

Marginal Spectrum for the Ensemble Empirical Mode Decomposition 


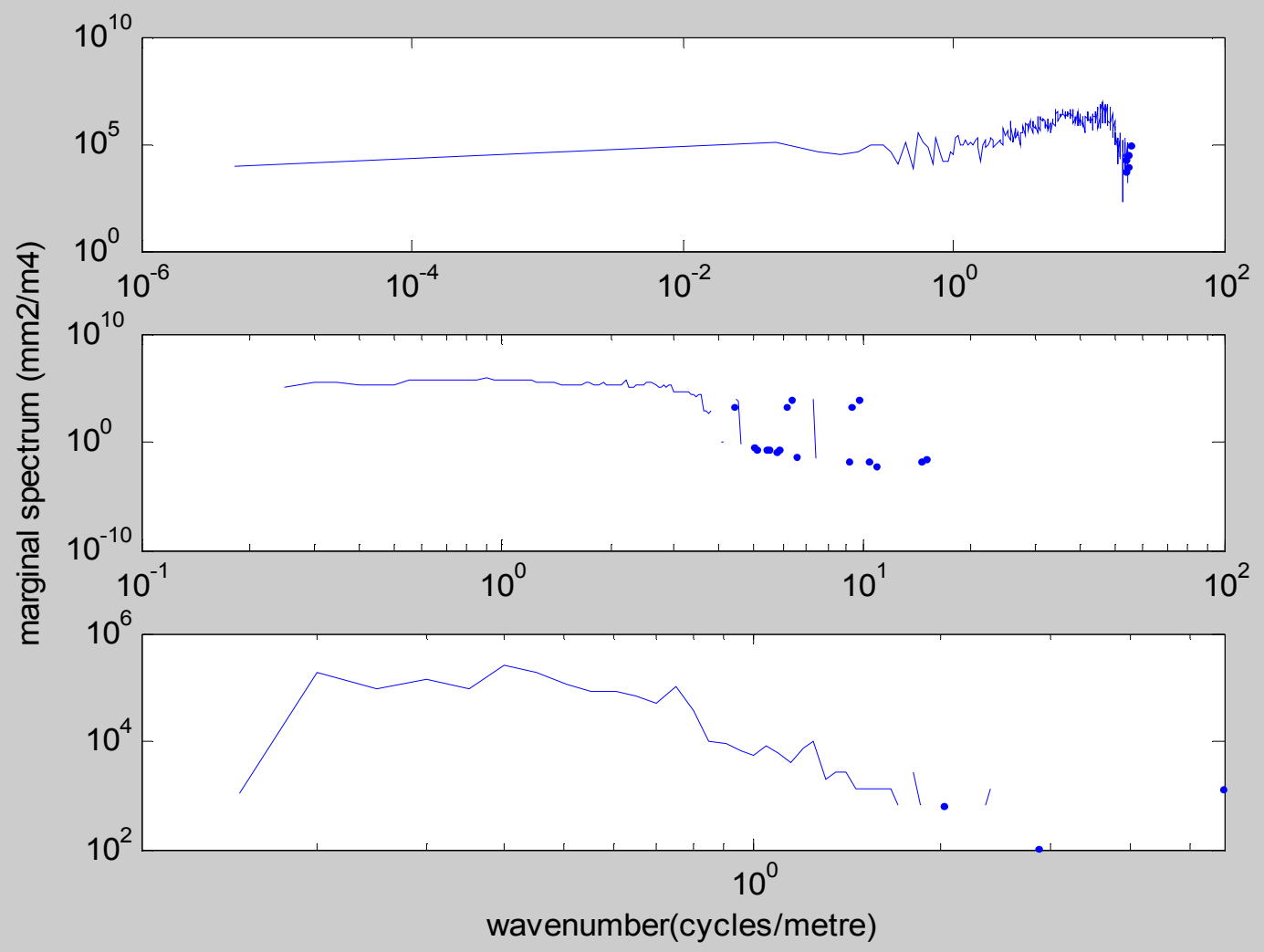

Marginal Spectrum for the Empirical Mode Decomposition 


\section{APPENDIX II}

\section{CEMD Energy-Phase relations}
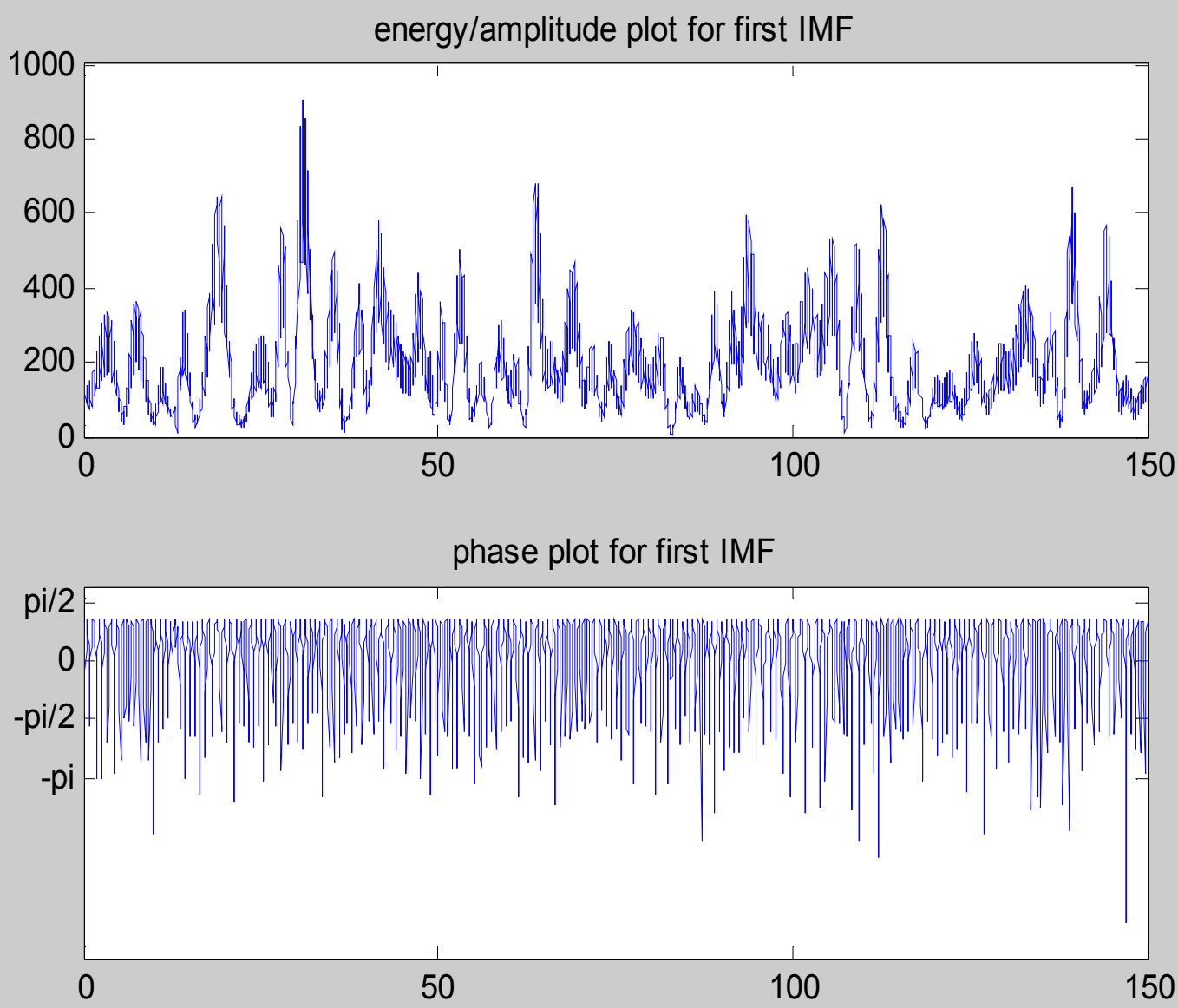

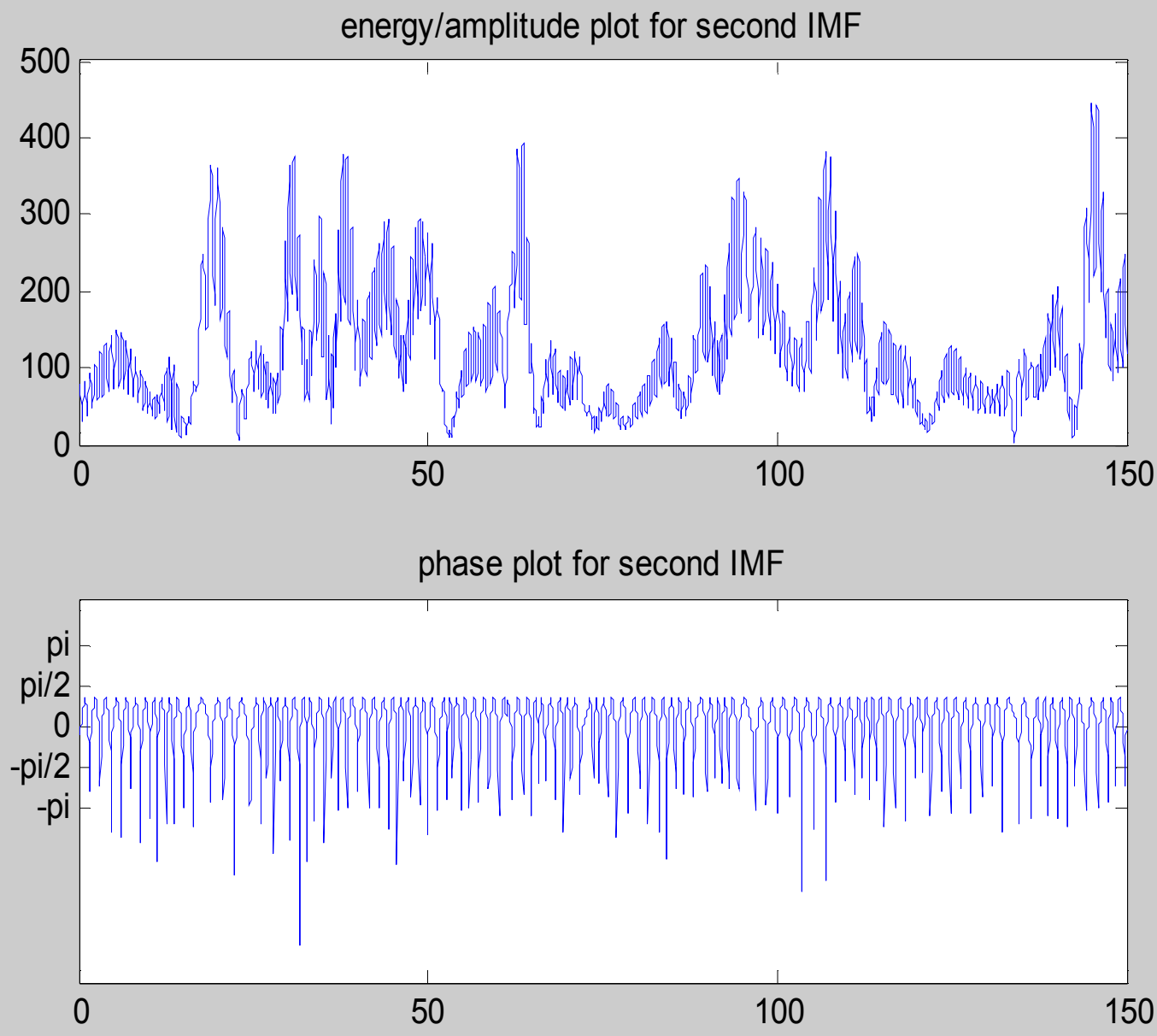


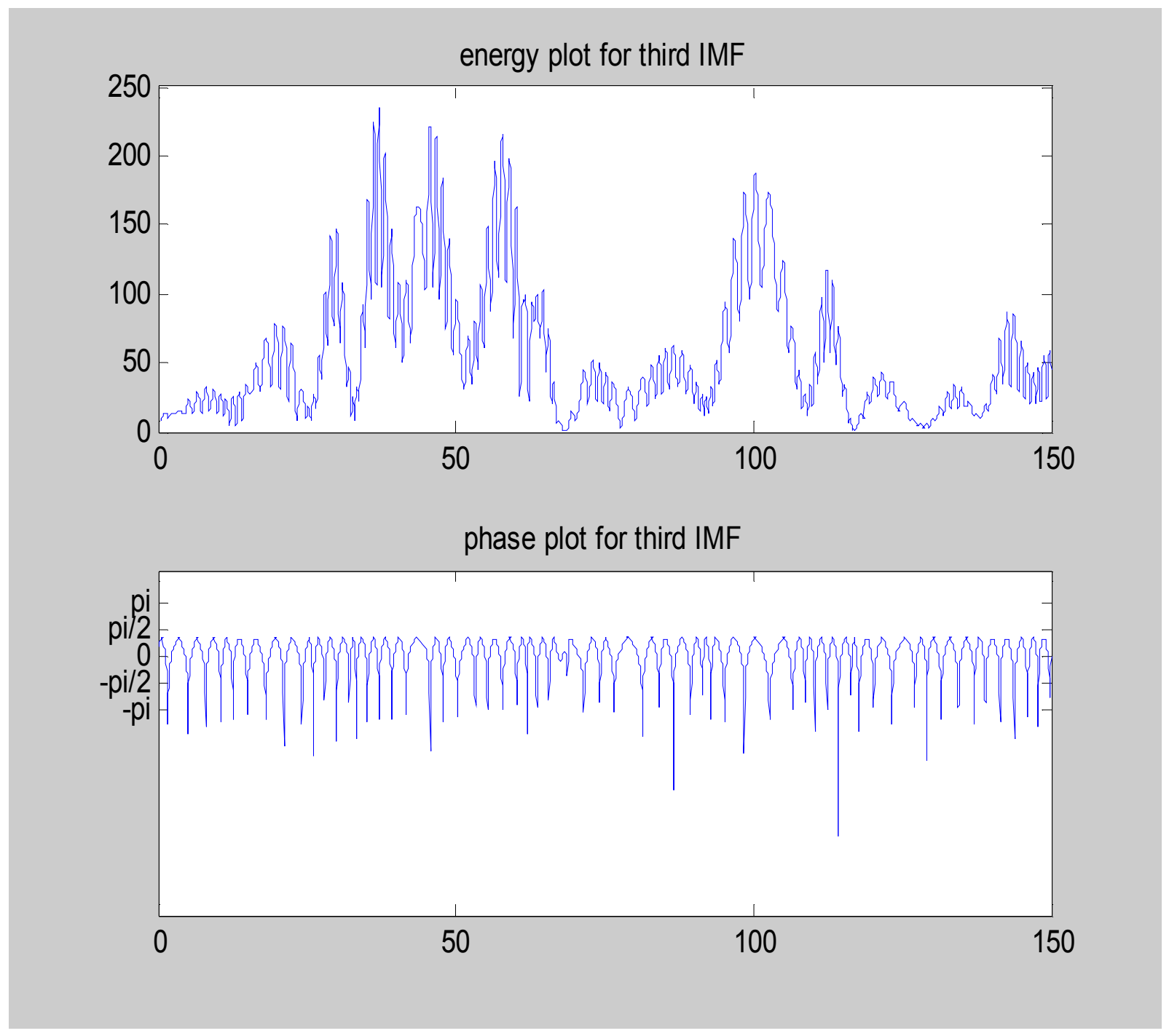




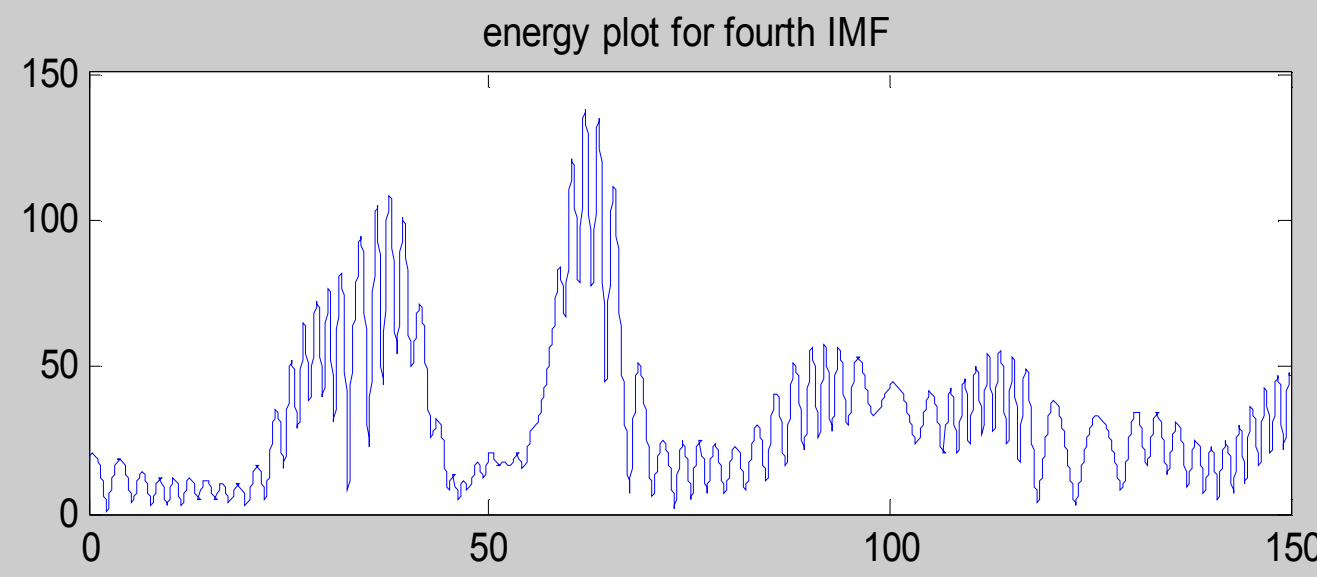

phase plot for fourth IMF

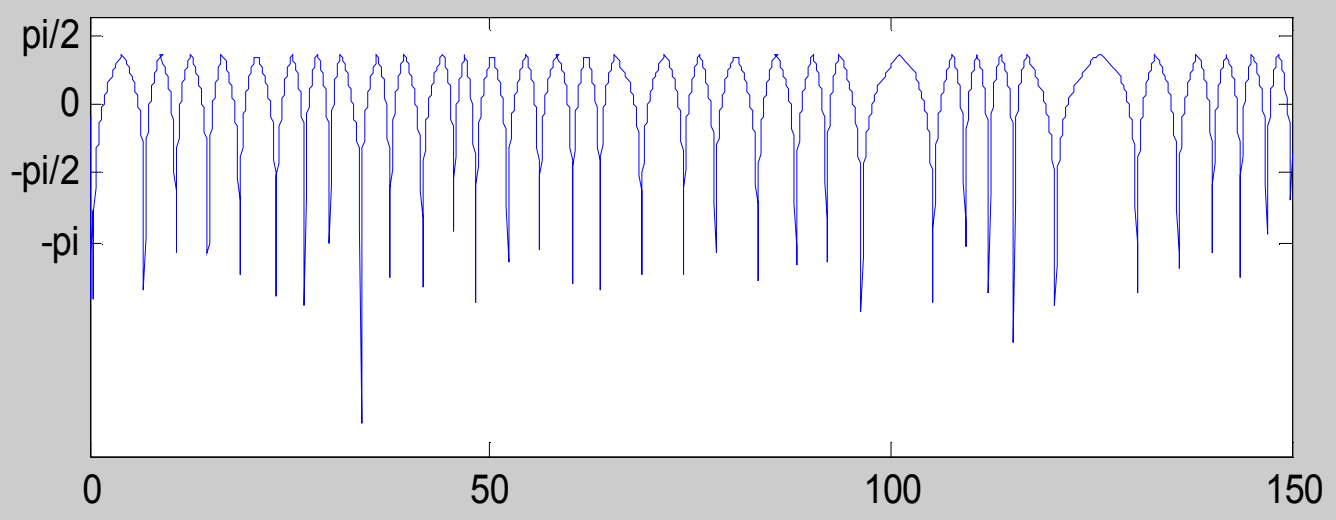



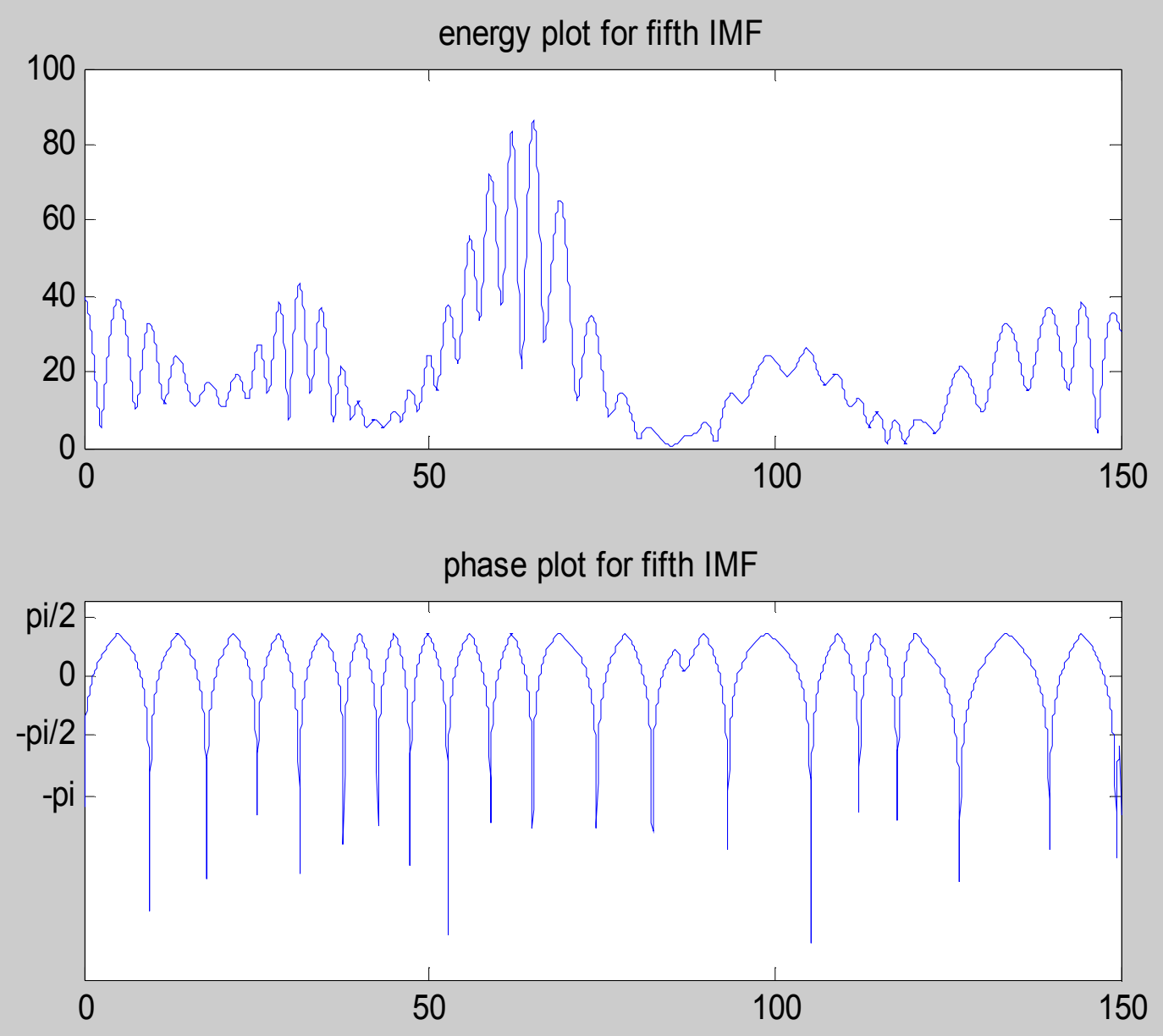


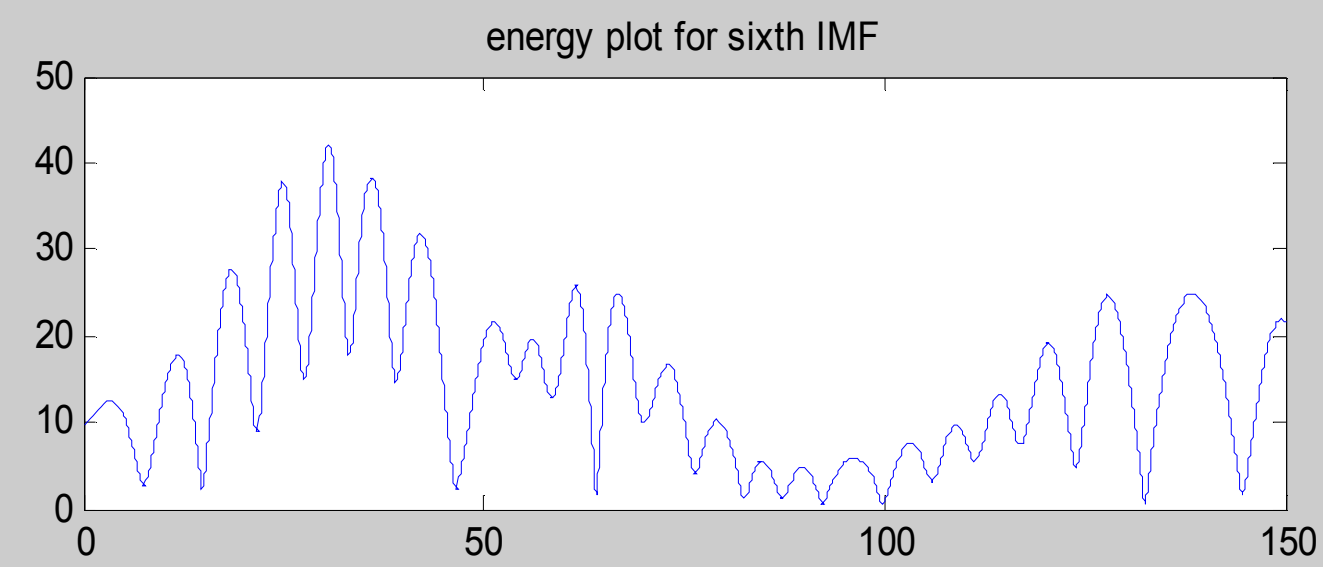

phase plot for sixth IMF

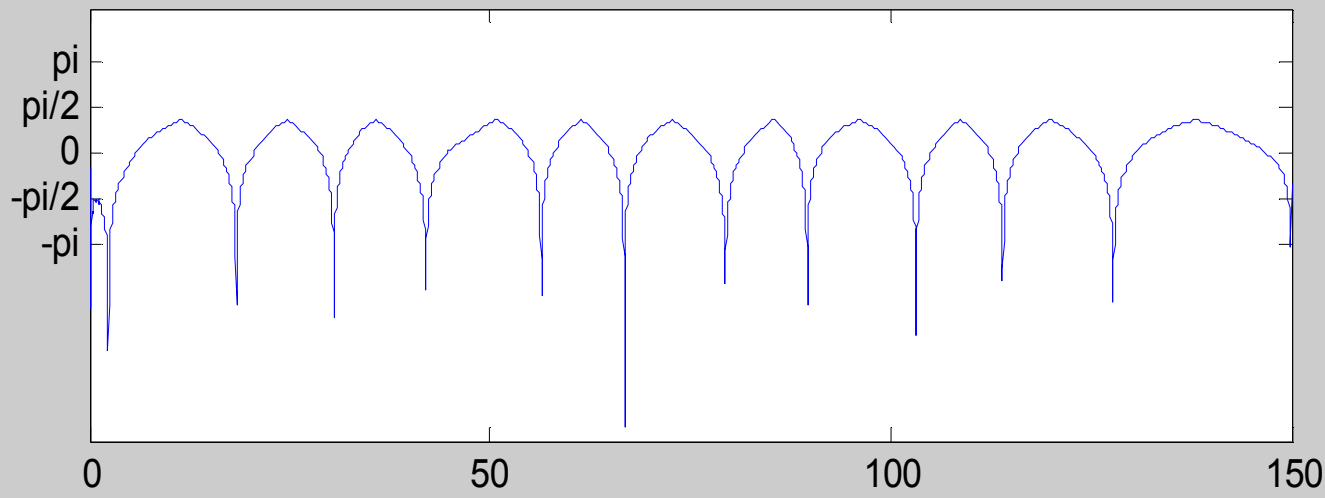




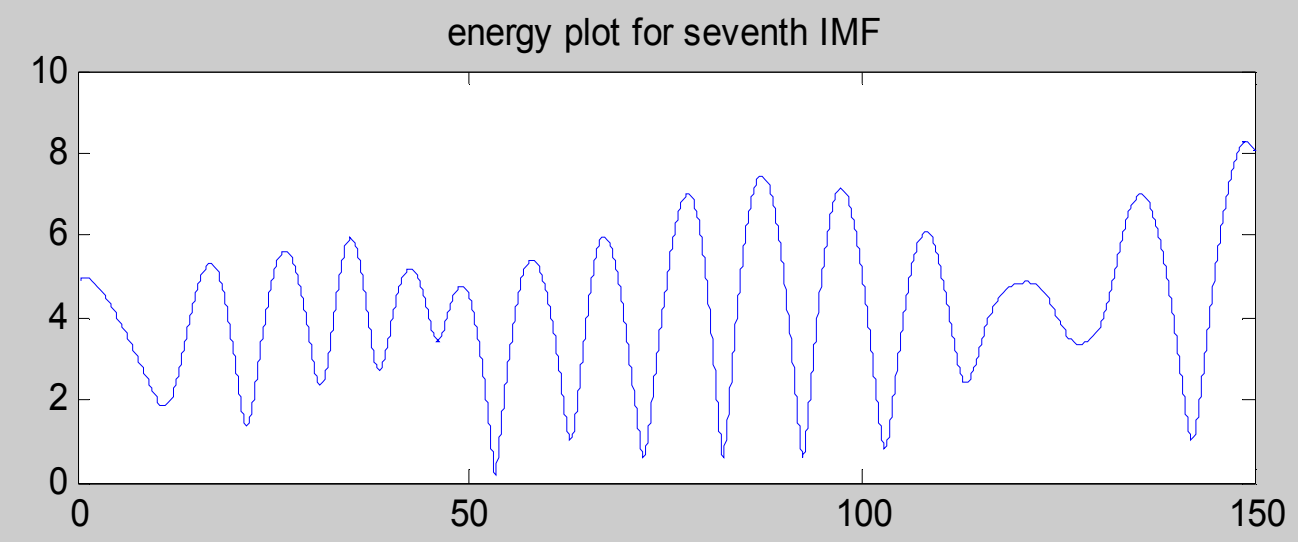

phase plot for seventh IMF

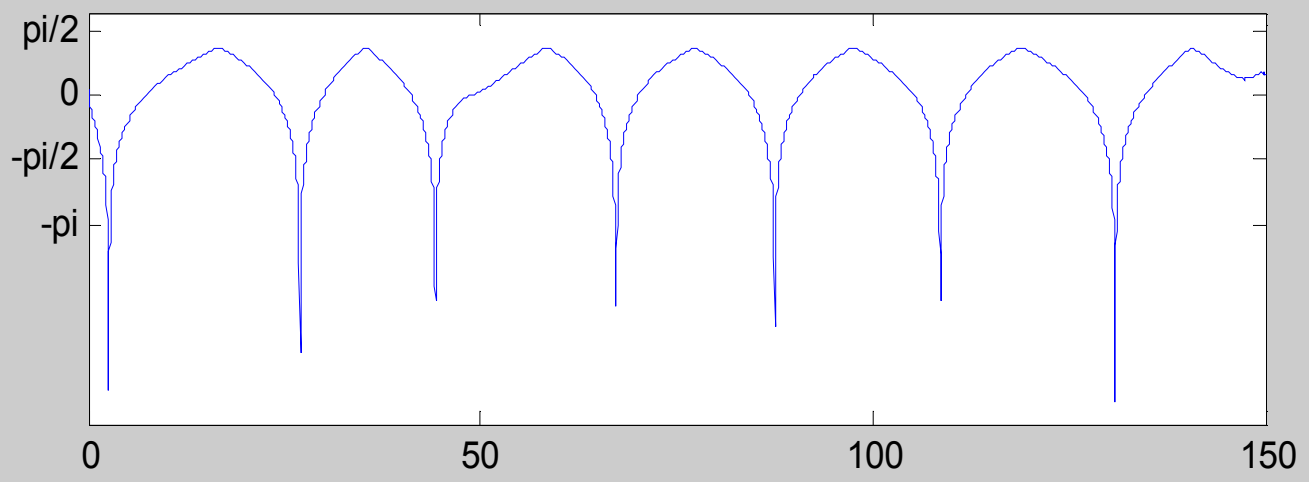




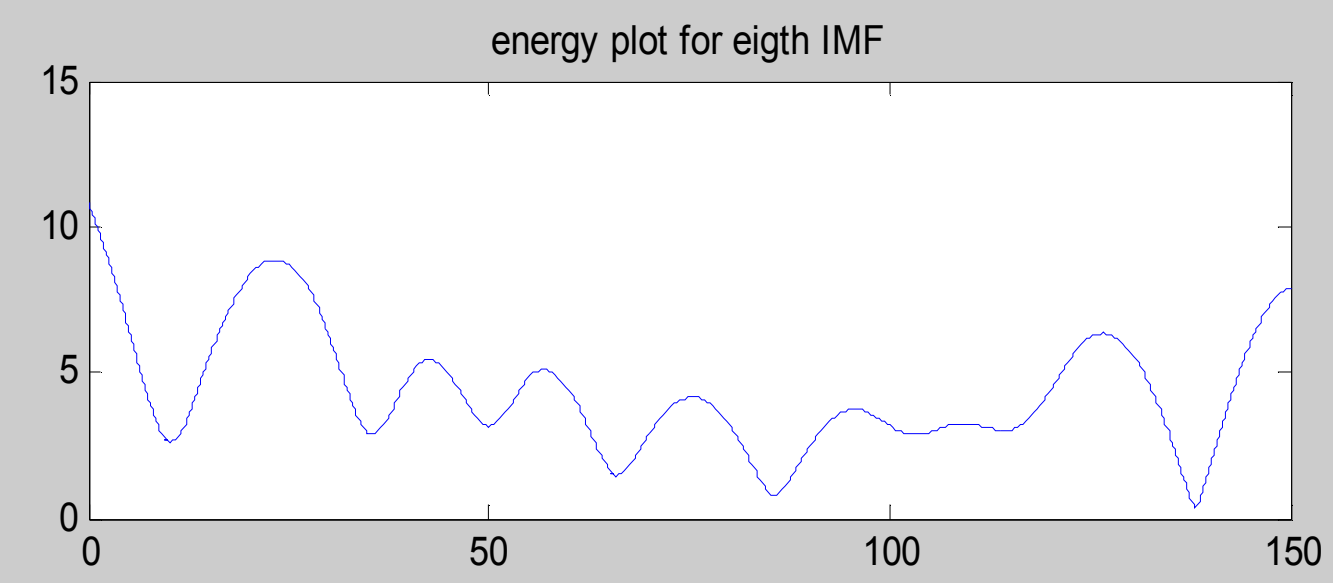

phase plot for eigth IMF

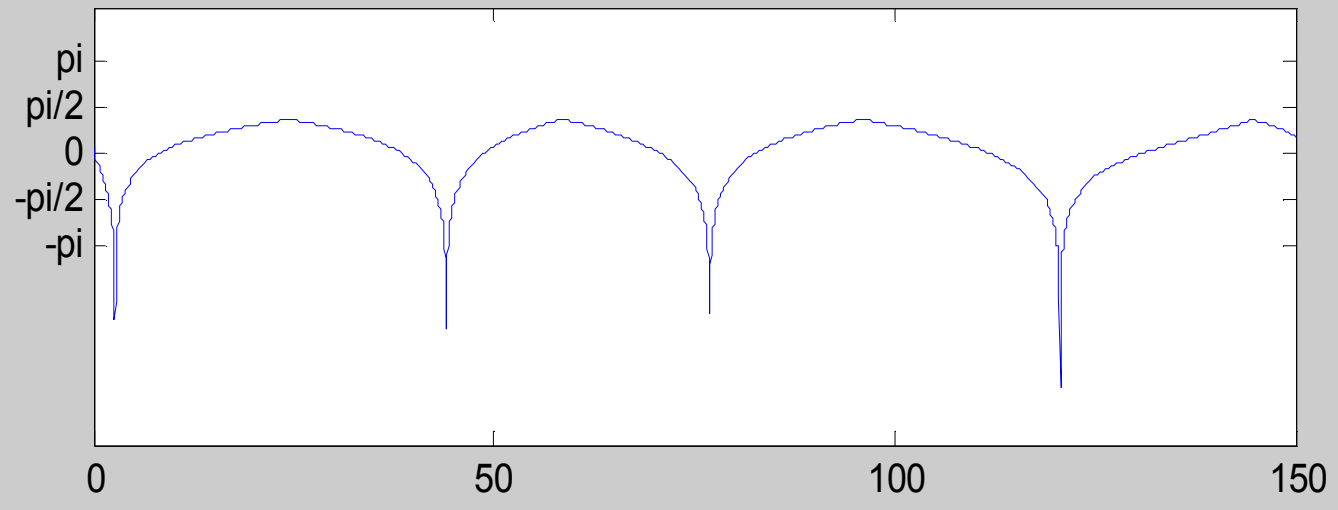




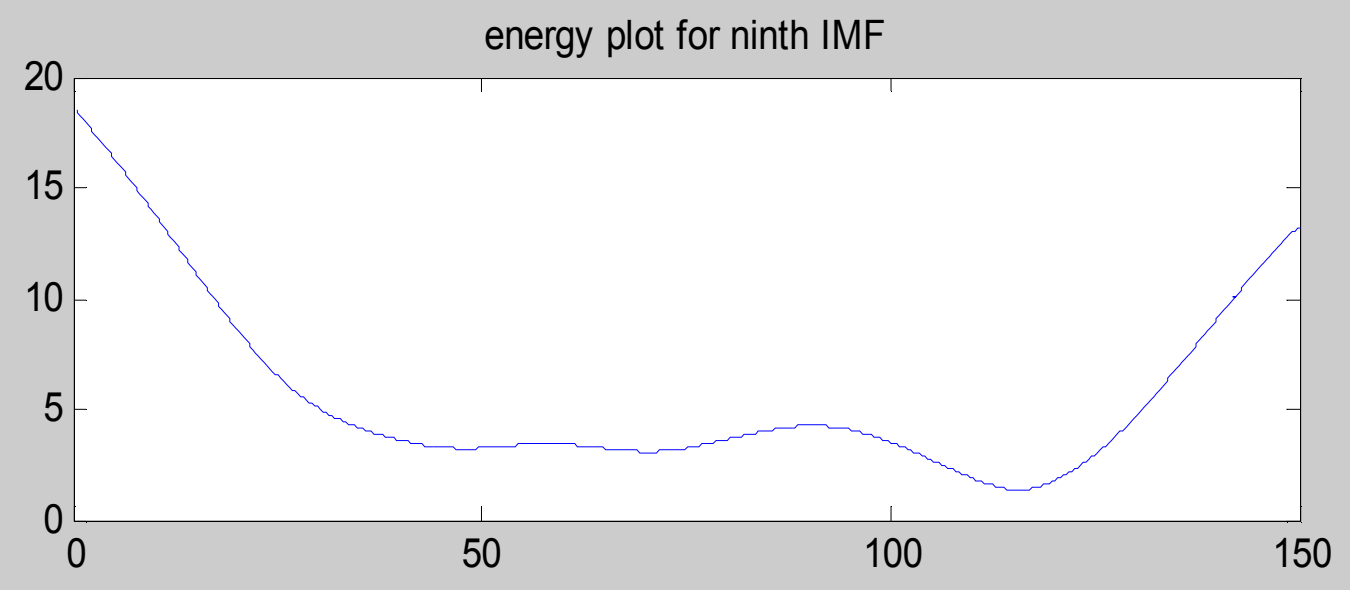

phase plot for ninth IMF

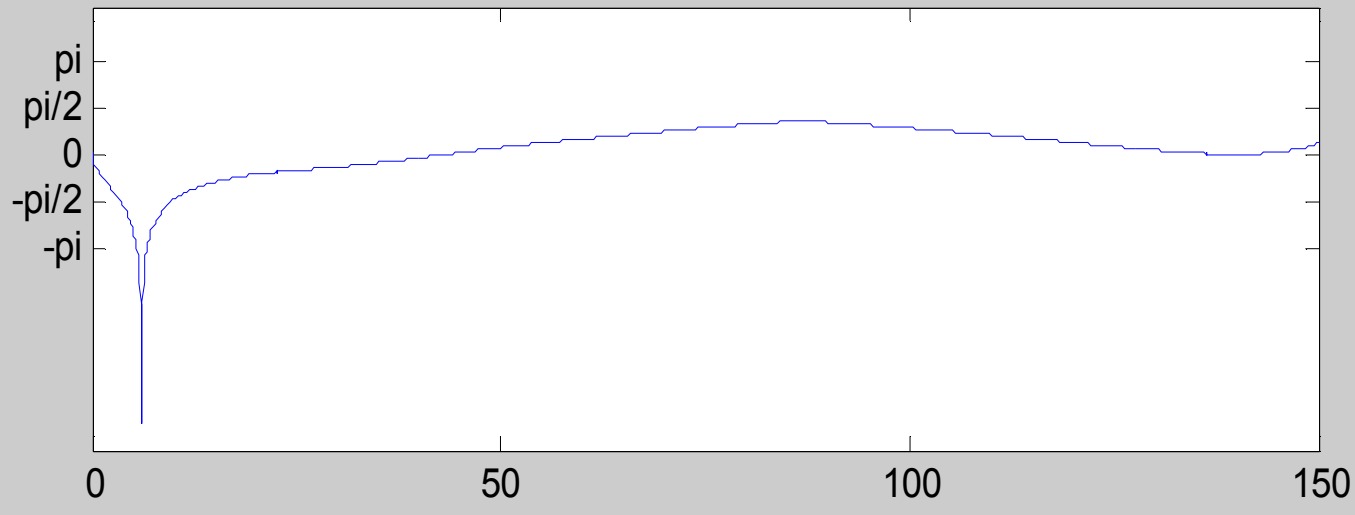



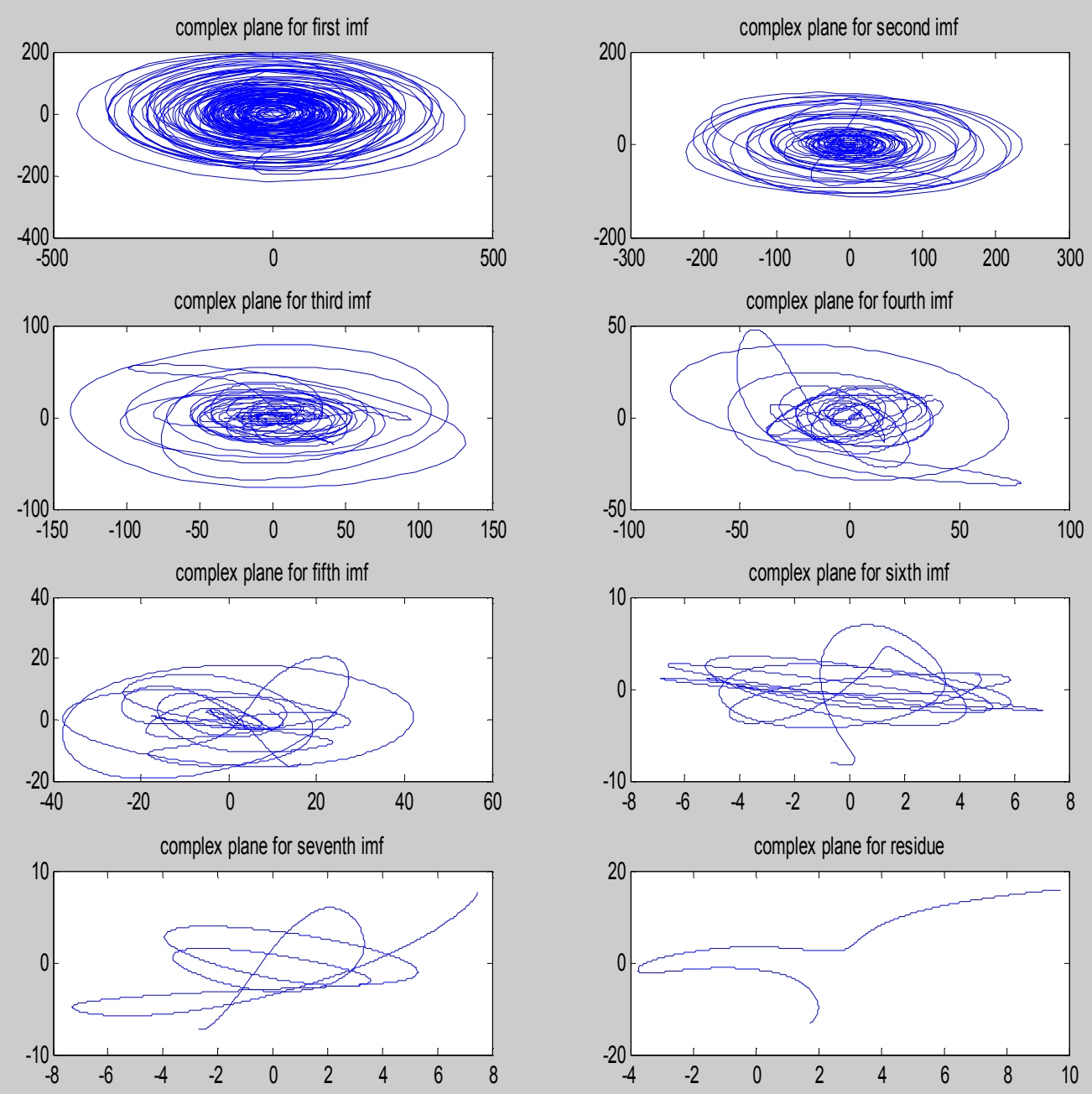


\section{APPENDIX III}

Phase Locking Plots For CEMD/EEMD

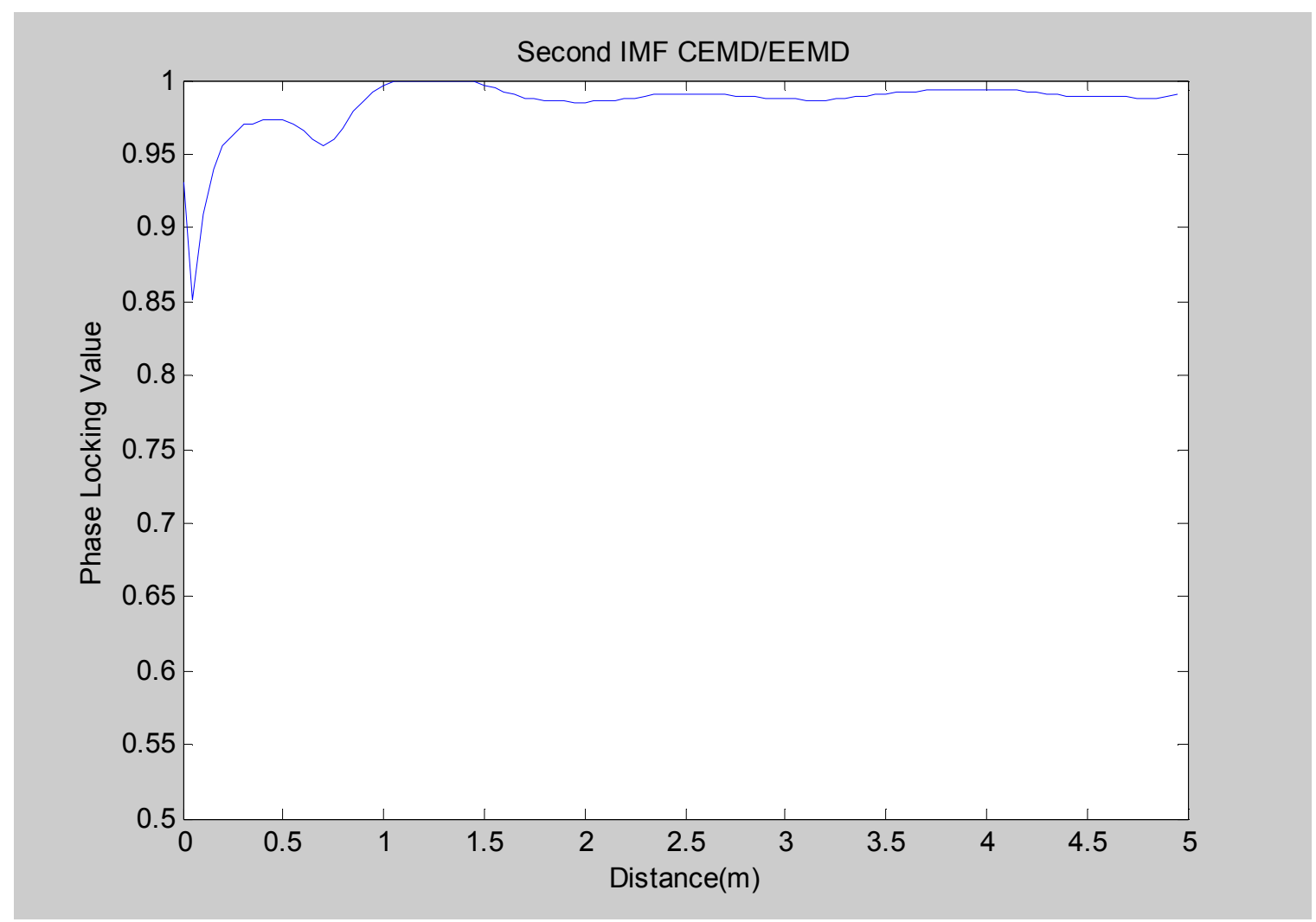



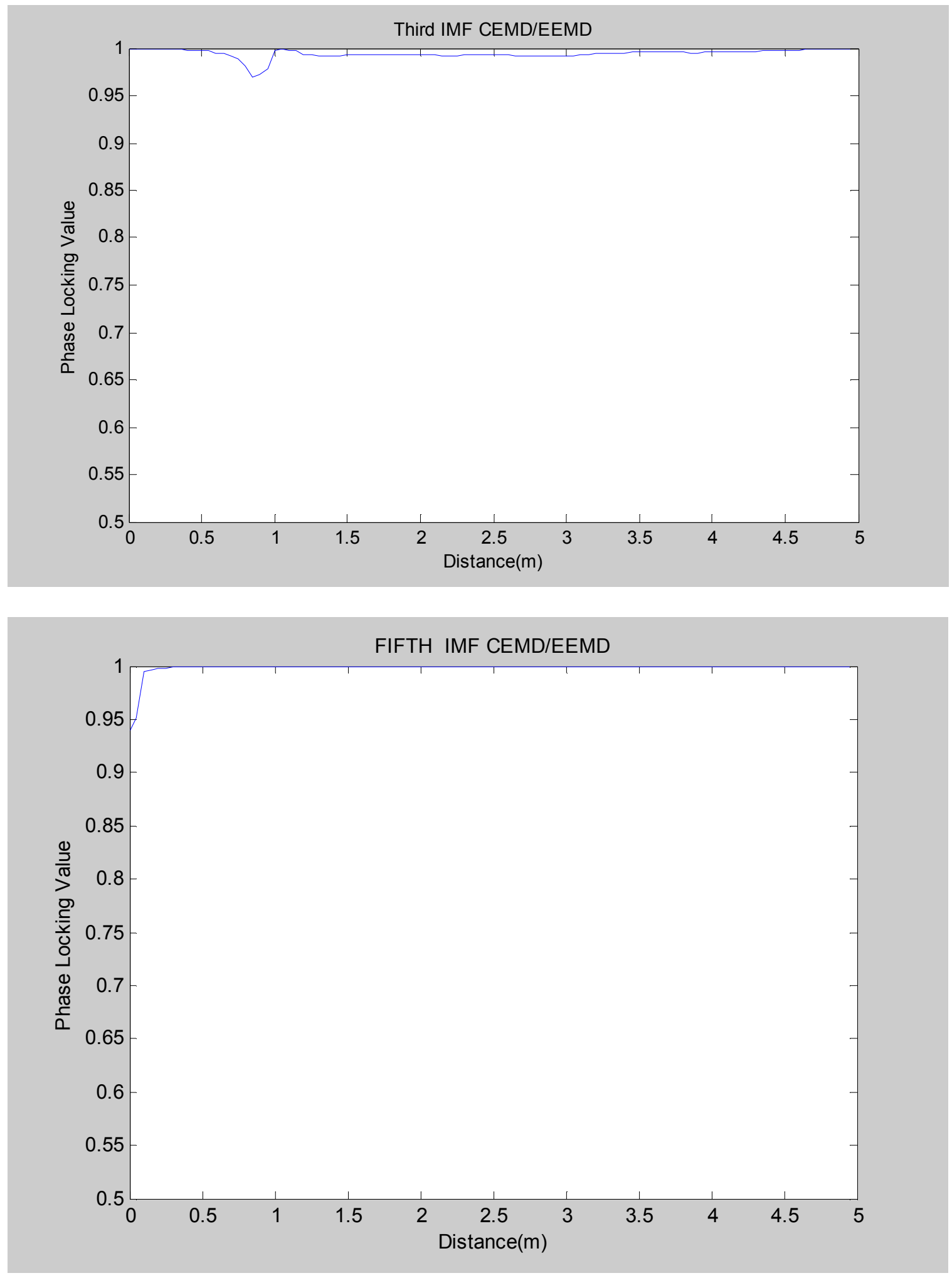
Phase Locking Plots for EMD/EEMD

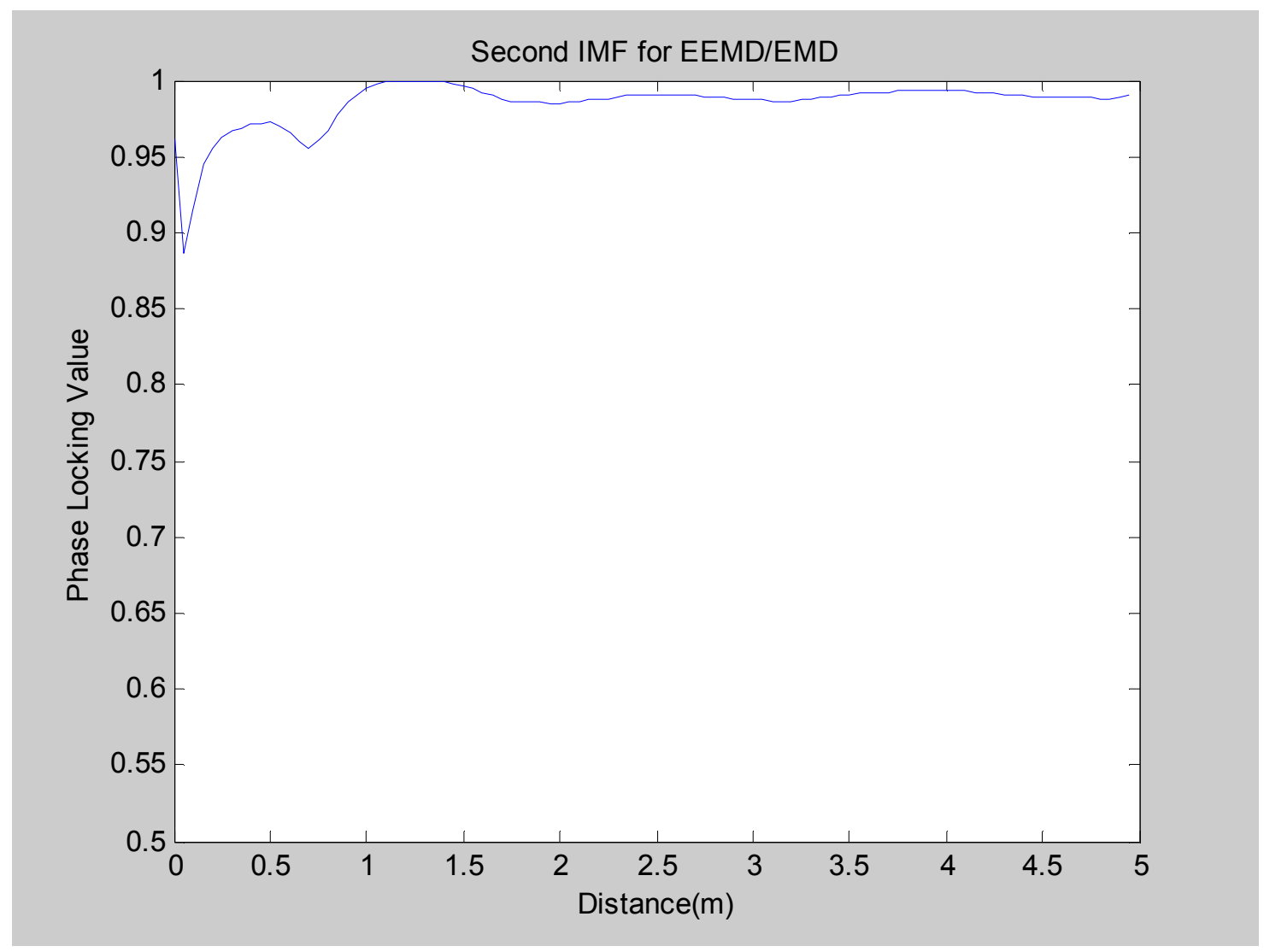




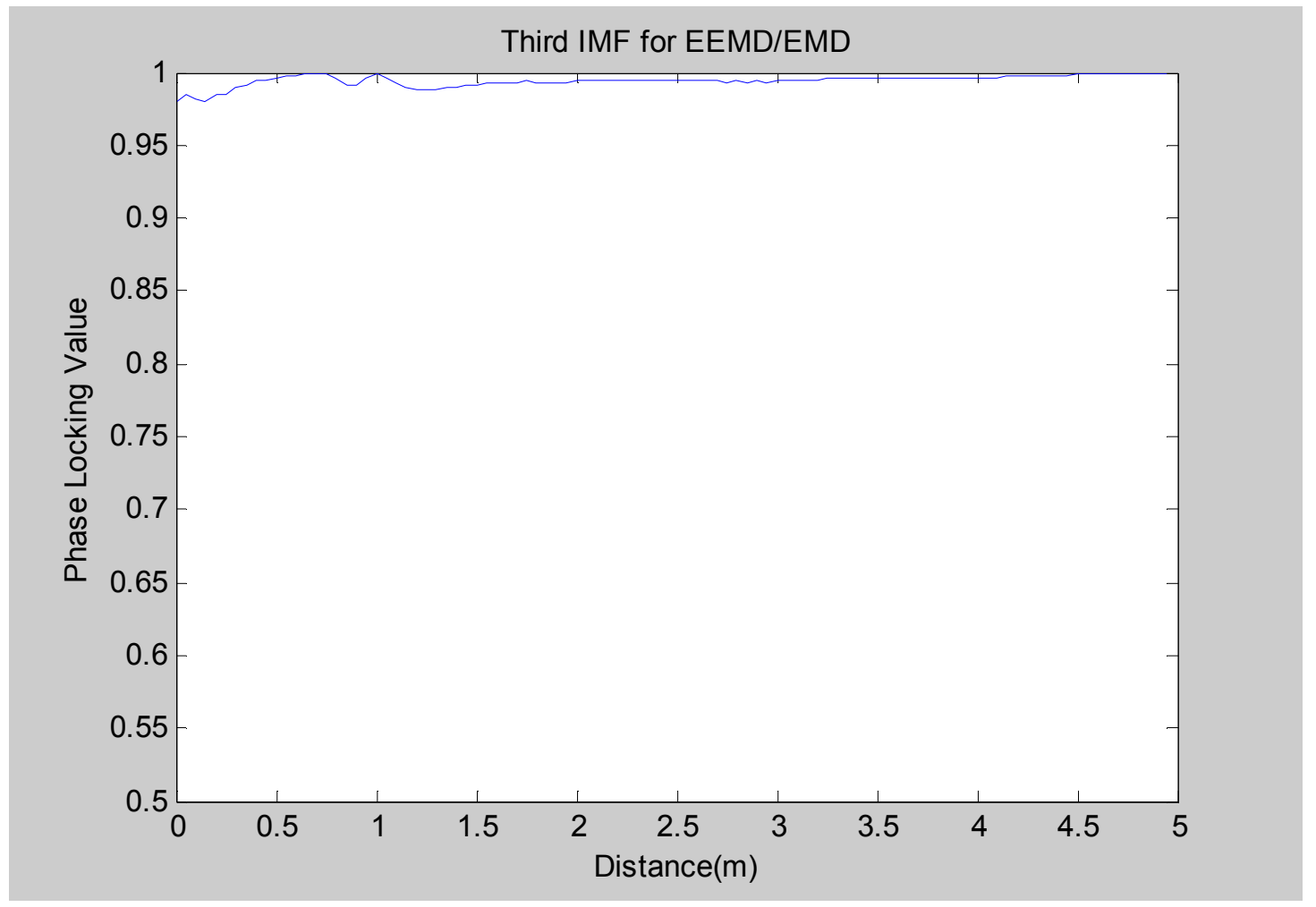




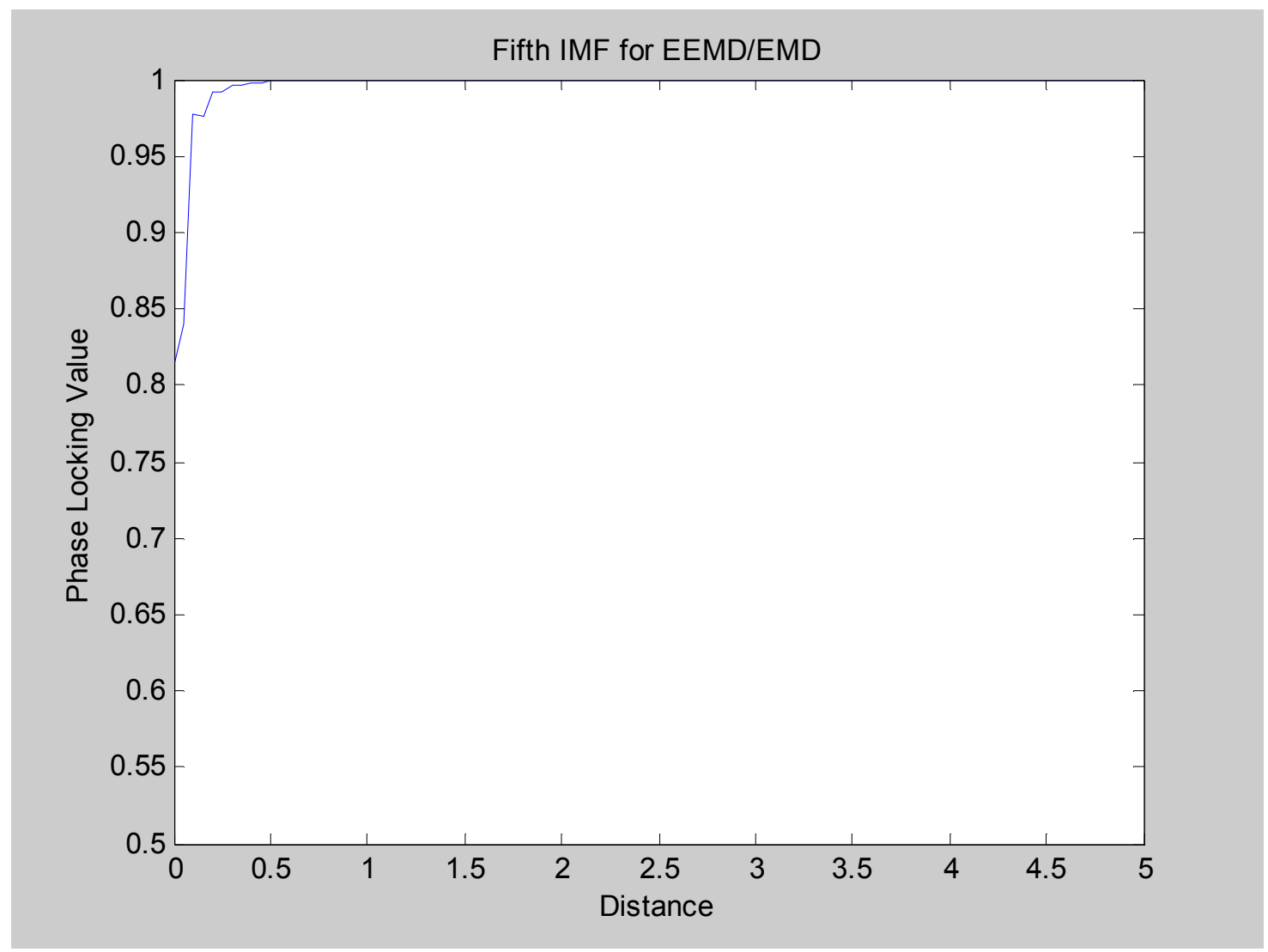

